Marcelo Chaves Silva

\title{
Problemas Isoperimétricos no Plano de Minkowski
}

Dissertação apresentada como requisito parcial para obtenção do grau de Mestre pelo Programa de Pós-graduação em Matemática do Departamento de Matemática da PUC-Rio

Orientador: Prof. Marcos Craizer 
Marcelo Chaves Silva

\title{
Problemas Isoperimétricos no Plano de Minkowski
}

Dissertação apresentada como requisito parcial para obtenção do grau de Mestre pelo Programa de Pós-graduação em Matemática do Departamento de Matemática do Centro Técnico Científico da PUC-Rio. Aprovada pela Comissão Examinadora abaixo assinada.

\author{
Prof. Marcos Craizer \\ Orientador \\ Departamento de Matemática - PUC-Rio
}

Prof. Ralph Costa Teixeira Instituto de Matemática - UFF

Prof. Victor Augusto Giraldo Instituto de Matemática - UFRJ

Prof. Rodrigo Pereira Pacheco

ICEx, Departamento de Matemática - UFF

Prof. José Eugenio Leal

Coordenador Setorial do Centro Técnico Científico - PUC-Rio 
Todos os direitos reservados. É proibida a reprodução total ou parcial do trabalho sem autorização da universidade, do autor e do orientador.

\section{Marcelo Chaves Silva}

Graduou-se em Licenciatura em Matemática na Universidade Federal do Rio de Janeiro. Defendeu seu primeiro mestrado em Ensino de Matemática também na Universidade Federal do Rio de Janeiro com a dissertação Um Estudo Qualitativo dos Efeitos de Descrições do Comportamento no Infinito de Funções Racionais. Trabalha no Centro Federal de Educação Tecnológica Celso Suckow da Fonseca, o CEFET-RJ, desde 2008 como professor do Ensino Básico Técnico e Tecnológico.

Ficha Catalográfica

Silva, Marcelo Chaves

Problemas Isoperimétricos no Plano de Minkowski / Marcelo Chaves Silva; orientador: Marcos Craizer. - Rio de Janeiro : PUC-Rio, Departamento de Matemática, 2015.

v., 70 f: il. ; $29,7 \mathrm{~cm}$

1. Dissertação (mestrado) - Pontifícia Universidade Católica do Rio de Janeiro, Departamento de Matemática.

Inclui referências bibliográficas.

1. Matemática - Tese. 2. Plano Normado. 3. Norma Dual. 4. Desigualdade de Minkowski. 5. Geometria Diferencial. I. Craizer, Marcos. II. Pontifícia Universidade Católica do Rio de Janeiro. Departamento de Matemática. III. Título. 


\section{Agradecimentos}

A Deus, pois Ele é a causa de todas as coisas.

Ao meu orientador Marcos Craizer por seus valiosos ensinamentos, por sua paciência e, principalmente, por ter acreditado em mim em todos os momentos.

À minha esposa Luciene pela compreensão e parceria indispensáveis do dia a dia.

Ao meu filho Gustavo pelas muitas horinhas de felicidade.

À minha mãe dona Mercedes por orar e torcer por mim em todos os momentos de minha vida.

Ao meu pai Sr. Elizeu pelo exemplo de vida que sempre tentarei seguir (in memoriam).

Ao professor Victor Giraldo por ser primordial em minha formação como professor de Matemática.

Ao professor Ivo Fernandez Lopez pelo apoio e pela inspiração (in memoriam).

Às minhas colegas Dania e Yunelsy pela força inestimável durante nossa caminhada.

Ao pessoal do departamento de Matemática pela ajuda constante, em especial à Creuza.

À PUC-Rio pela oportunidade de estudar em um ambiente com pessoas de tanto valor. 


\section{Resumo}

Silva, Marcelo Chaves; Craizer, Marcos. Problemas Isoperimétricos no Plano de Minkowski. Rio de Janeiro, 2015. 70p. Dissertação de Mestrado - Departamento de Matemática, Pontifícia Universidade Católica do Rio de Janeiro.

O objetivo principal deste trabalho é resolver o problema isoperimétrico no plano de Minkowski, isto é, determinar dentre todas as curvas convexas, fechadas, simples e suaves de perímetro fixo de um plano munido com uma norma qualquer, qual é aquela que delimita a maior área. Mostraremos que a solução para este problema não é necessariamente o círculo como no caso euclideano e sim uma curva conhecida como isoperimetrix. Para isto, vamos demonstrar a desigualdade de Minkowski a partir do conceito de área mista. Em seguida, vamos determinar se há outros casos (além do caso euclideano) em que o círculo coincide com o isoperimetrix. Também iremos mostrar que o perímetro da bola nestes planos pode assumir qualquer valor real entre seis e oito, sendo seis quando a bola for um hexágono regular afim e oito quando for um paralelogramo.

\section{Palavras-chave}

Plano Normado; Norma Dual; Desigualdade de Minkowski; Geometria Diferencial. 


\section{Abstract}

Silva, Marcelo Chaves; Craizer, Marcos (Advisor). Isoperimetric Problems in the Minkowski Plane. Rio de Janeiro, 2015. 70p. MSc. Dissertation - Departamento de Matemática, Pontifícia Universidade Católica do Rio de Janeiro.

The main objective of this work is to solve the isoperimetric problem in the Minkowski plane, i. e., determine among all smooth simple closed convex curves of a normed plane with fixed perimeter, what is that which defines the largest area. We will show that the solution to this problem is not necessarily the circle as in the Euclidean case, but a curve known as isoperimetrix. For this, we will demonstrate the Minkowski inequality from the concept of mixed area. Then, we determine if there are other cases (apart from the Euclidean case) in which the circle coincides with the isoperimetrix. We will also show that the ball perimeter in a normed plane can take any real value between six and eight. It is six when the ball is an affine regular hexagon and eight when it is a parallelogram.

\section{Keywords}

Normed Plane; Dual Norm; Minkowski Inequality; Differential Geometry. 


\section{Sumário}

$\begin{array}{lll}1 & \text { Introdução } & 10\end{array}$

2 Referencial teórico $\quad 12$

2.1 Convexidade 12

2.2 Espaços vetoriais normados 13

2.3 Aplicações contínuas 22

2.4 Curvas planas 23

$\begin{array}{ll}2.5 \text { A função suporte } & 25\end{array}$

3 A desigualdade de Minkowski $\quad 30$

4 O plano de Minkowski 40

4.1 Bolas no plano de Minkowski 40

4.2 O dual 46

4.3 Normalidade no plano de Minkowski 52

4.4 O problema isoperimétrico 54

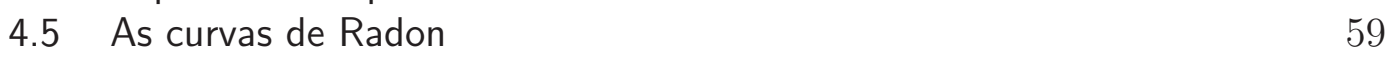

5 O perímetro da bola unitária no plano de Minkowski $\quad 61$

$\begin{array}{lll}6 & \text { Referências Bibliográficas } & \mathbf{7 0}\end{array}$ 


\section{Lista de figuras}

2.1 Projeção ortogonal de $v$ sobre a reta que contém $\hat{u}$. 21

2.2 Círculo de raio $\mathrm{R}$ e centro na origem. 25

2.3 Função suporte. 26

$\begin{array}{ll}2.4 & \text { As tangentes à } c \text { envelopam } c . \\ & 29\end{array}$

3.1 As curvas $c_{0}$ e $c_{1}$ têm a mesma normal nos pontos $\alpha_{0}$ e $\alpha_{1}$ respectivamente. $\quad 30$

3.2 Translação da curva $c_{1}$ tal que o seu centro fique sobre a curva $c_{0} . \quad 31$

3.3 Translação da curva $c_{1}$ tal que ela tangencie a curva $c_{0}$. 33

3.4 Translações de $r_{0} K_{1}$ e $R_{0} K_{1}$. 35

3.5 Duas curvas podem ter infinitos pontos de interseção. 35

3.6 Translações de $r c_{1}$ que tangenciam externamente a curva $c_{0} . \quad 36$

3.7 Lugar geométrico das origens transladadas de modo que $r c_{1}$ contenha o ponto $\alpha_{0}$. 37

3.8 Os centros das translações de $r c_{1}$ pertencem às duas faixas hachuradas.

4.1 Círculos unitários para normas $\ell_{p}$. 41

4.2 Dois vetores tais que $\|x\|_{2}=\|y\|_{2}$, mas $\|x\|_{B} \neq\|y\|_{B}$. 43

4.3 Diferentes unidades de comprimento para diferentes direções. 44

4.4 A distância entre dois pontos. 45

4.5 Exemplos de bolas. 46

4.6 Um exemplo para o enunciado do teorema 4.7. 53

4.7 O isoperimetrix da elipse. 58

4.8 Construção de uma curva de Radon. 60

5.1 Construção de um hexágono de lados unitários. 61

5.2 A curva $c$ está inscrita em $\mathcal{P}$. 63

5.3 Hexágono regular afim. 64

5.4 O hexágono está contido em $\partial B$. 65

$5.5 \overline{x_{2}\left(x_{2}-x_{1}\right)} \subseteq \partial B . \quad 66$

5.6 O paralelogramo está contido em $\partial B$. 67

5.7 Exemplo de bola cujo perímetro é $6+2 \xi$. 69 
Felicidade se acha é em horinhas de descuido.

Guimarães Rosa, Tutaméia - Terceiras Estórias. 


\section{Introdução}

Neste trabalho concentraremos nosso estudo em espaços reais de Banach de duas dimensões, também denominados planos normados ou planos de Minkowski. A Geometria destes planos - e, mais geralmente, a geometria de espaços reais de Banach de dimensão finita - é denominada por Thompson [1] como Geometria de Minkowski. Lembramos que este termo não deve ser confundido com a Geometria de Espaço-Tempo de Minkowski. Segundo Martini \& $\mathrm{Wu}[2]$, a Geometria de Minkowski tem um pouco mais de cem anos e, através da história, está relacionada a nomes como: Riemann, Minkowski, Banach, Day, Busemann, Amir, Klee, Grünbaum e outros. Diversas contribuições foram dadas por eles em áreas como Geometria elementar, Geometria diferencial, Geometria convexa e Geometria computacional. Uma delas será um dos focos de nossa pesquisa e será enunciada a seguir.

O problema isoperimétrico clássico consiste em determinar, dentre todas as curvas simples e fechadas de perímetro fixo, qual é aquela que delimita a maior área. Na Geometria euclideana, é bem conhecido que a solução para este problema é o círculo. Demonstrações para este fato podem ser encontradas em Alencar \& Santos [3] e Carmo [4]. Contudo, é curioso notar que o caso euclideano parece mascarar a verdadeira resposta para esta questão. Busemann [5] foi o primeiro a descobrir que uma curva denominada isoperimetrix é a que satisfaz as condições do problema isoperimétrico para planos munidos com uma norma arbitrária. Para isto, Busemann usou o teorema de Brunn-Minkowski, que aqui será simplesmente denominado por desigualdade de Minkowski. Ele também resolveu o problema para espaços de dimensão finita.

Posteriormente houve abordagens diferentes para a resolução do problema isoperimétrico, como a feita por Strang [6], que usa a teoria de cálculo das variações.

Nesta pesquisa, resolveremos o problema isoperimétrico no plano de Minkowski estritamente para curvas convexas e suaves. Esta restrição se deve primeiramente pelo fato intuitivo de que, se uma curva é não-convexa, sempre podemos aumentar a área sem aumentar o perímetro, logo, a solução deve ser uma curva convexa. Depois porque curvas não-suaves podem ser aproximadas 
por curvas suaves.

A outra escolha que precisamos fazer é a área considerada. De acordo com Busemann [5], como a área de Minkowski e a área euclideana diferem apenas por um fator constante, a solução para a nossa questão é a mesma se considerarmos qualquer uma destas duas áreas. Portanto, em todo o presente trabalho, quando nos referirmos a área de uma região, estaremos considerando a área usual.

Além do que foi exposto acima, também abordaremos os seguintes tópicos. No capítulo 2, faremos um breve resumo de conceitos e resultados básicos que serão importantes para o entendimento dos capítulos seguintes. No capítulo 3, definiremos área mista e demonstraremos a desigualdade de Minkowski, que será fundamental na prova do problema isoperimétrico. No capítulo 4, iremos resolver o problema isoperimétrico para planos munidos com uma norma qualquer e também determinar quando a solução coincide com a bola unitária, assim como ocorre no caso euclideano. Enfim, no capítulo 5, estimaremos todos os possíveis valores para o perímetro da bola unitária. 


\section{2 \\ Referencial teórico}

Neste capítulo iremos abordar alguns dos conceitos básicos e fundamentais para o entendimento dos capítulos subsequentes. Na seção 2.1, abordaremos o conceito de conjunto convexo e outras definições relacionadas com convexidade. Na seção 2.2, definiremos espaço vetorial e outras noções importantes como produto interno, norma e vetores ortogonais. Na seção 2.3, vamos definir funções contínuas e mostrar que toda transformação linear definida sobre um espaço vetorial de dimensão finita é contínua. Na seção 2.4, faremos um resumo da teoria de curvas planas. E finalmente na seção 2.5, definiremos função suporte que nos será muito útil, pois a partir dela poderemos obter fórmulas (convenientes em nosso contexto) para determinar: comprimento de curvas, área de regiões limitadas por estas curvas, a área mista e também a própria curva de maneira única.

Segundo Thompson [1], definimos:

Definição 2.1 Um conjunto $K$ é dito ser simétrico com relação à origem quando $(-1) K=K$.

O termo simétrico será mencionado muitas vezes neste trabalho e sempre será sinônimo de simétrico com relação à origem.

\section{1}

\section{Convexidade}

Veremos no capítulo 4 que há uma correspondência biunívoca entre normas (conceito que determina como medimos distâncias) e conjuntos convexos, fechados, limitados e simétricos com interior não-vazio. Isto faz da convexidade uma propriedade essencial em nossa pesquisa. As definições expostas a seguir podem ser encontradas em Thompson [1].

Definição 2.2 Um conjunto $C$ é chamado convexo quando, para quaisquer pontos $x_{1}$ e $x_{2}$ pertencentes a $C$, todos os pontos da forma $p=\alpha x_{1}+(1-\alpha) x_{2}$, em que $\alpha \in[0,1]$, pertencem a $C$. Isto é, dados quaisquer dois pontos $x_{1}$ e $x_{2}$ de $C$, o segmento de reta de extremidades $x_{1}$ e $x_{2}$ está contido em $C$. 
Definição 2.3 Um subconjunto convexo $S$ de um conjunto convexo $C$ é chamado um subconjunto extremo de $C$ quando, para todo $x \in S e$ $x=\alpha x_{1}+(1-\alpha) x_{2} \operatorname{com} x_{1}, x_{2} \in C$ e $\alpha \in(0,1)$, então $x_{1}, x_{2} \in S$.

Definição 2.4 Um ponto $x$ de $C$ tal que $\{x\}$ é um subconjunto extremo de $C$ é chamado um ponto extremo de $C$.

Exemplo 2.1 Os vértices e lados de um polígono convexo $\mathcal{P}$ são subconjuntos extremos de $\mathcal{P}$. Mais geralmente, os vértices, arestas e faces de um poliedro convexo são subconjuntos extremos deste.

Definição 2.5 Um conjunto convexo e fechado E é chamado estritamente convexo quando todo ponto da fronteira de E é ponto extremo.

Definição 2.6 Sejam $A$ e $X$ conjuntos tais que $A \subseteq X$. O fecho convexo do conjunto A é o menor conjunto convexo que contém $A$. É denotado por conv $A$ e é possivel mostrar que

$\operatorname{conv} A=\left\{y \in X: y=\sum_{i=1}^{n} \alpha_{i} x_{i}\right.$ tal que $\alpha_{i} \geq 0, \sum_{i=1}^{n} \alpha_{i}=1, x_{i} \in A, n$ arbitrário $\}$

\section{2}

\section{Espaços vetoriais normados}

Seja $X$ um conjunto. Segundo Lima [7], temos as seguintes definições:

Definição 2.7 A adição é uma operação que a cada par de elementos $a, b \in X$ associa um novo elemento $a+b \in X$, chamado a soma de a e $b$.

Definição 2.8 A multiplicação por um número real é uma operação que, a cada número $\alpha \in \mathbb{R}$ e a cada elemento $v \in X$ associa um novo elemento $\alpha v \in X$, chamado o produto de $\alpha$ por $v$.

Enfim, dizemos que o conjunto $X$ é um espaço vetorial quando nele estão definidas as duas operações: a adição e a multiplicação por um número real, que satisfazem, para quaisquer $\alpha, \beta \in \mathbb{R}$ e $u, v, w \in X$, as condições abaixo:

Comutatividade: $u+v=v+u$;

Associatividade: $(u+v)+w=u+(v+w)$ e $(\alpha \beta) v=\alpha(\beta v)$;

Vetor nulo: Existe um vetor $0 \in X$, chamado vetor nulo, ou vetor zero, tal que $v+0=0+v=v$ para todo $v \in X$ 
Inverso aditivo: Para cada vetor $v \in X$ existe um vetor $-v \in X$, chamado o inverso aditivo, ou o simétrico de $v$, tal que $-v+v=v+(-v)=0$;

Distributividade: $(\alpha+\beta) v=\alpha v+\beta v$ e $\alpha(u+v)=\alpha u+\alpha v$;

Multiplicação por 1: $1 \cdot v=v$.

Os elementos de $X$ são chamados vetores.

Exemplo 2.2 O conjunto $\{0\}$ é um exemplo trivial de espaço vetorial.

Exemplo 2.3 Dado $n \in \mathbb{N}$, o conjunto $\mathbb{R}^{n}$ é um espaço vetorial. Em particular para $n=2$, temos o caso que será o foco desta pesquisa: o plano. Os elementos de $\mathbb{R}^{2}$ são os pares ordenados $u=\left(u_{1}, u_{2}\right)$ e $v=\left(v_{1}, v_{2}\right)$ de números reais. $A$ igualdade vetorial $u=v$ ocorre quando, por definição, $u_{1}=v_{1}$ e $u_{2}=v_{2}$. As operações são definidas assim:

$$
u+v=\left(u_{1}+v_{1}, u_{2}+v_{2}\right)
$$

$$
\alpha u=\left(\alpha u_{1}, \alpha u_{2}\right)
$$

$O$ vetor zero é $0=(0,0)$. O inverso aditivo de $u$ é $-u=\left(-u_{1},-u_{2}\right)$. É fácil verificar que $\mathbb{R}^{2}$ munido destas operações e definições acima é um espaço vetorial.

Definição 2.9 Sejam E, F espaços vetoriais. Uma transformação linear $A: E \rightarrow F$ é uma correspondência que associa a cada vetor $v \in E$ um vetor $A(v)=A \cdot v=A v \in F$ de modo que, para quaisquer $u, v \in E$ e $\alpha \in \mathbb{R}$, sejam satisfeitas as seguintes propriedades:

$$
\begin{aligned}
& \text { 1. } A(u+v)=A u+A v ; \\
& \text { 2. } A(\alpha \cdot v)=\alpha \cdot A v .
\end{aligned}
$$

O vetor $A v$ chama-se a imagem de $v$ pela transformação $A$. A imagem de 0 por qualquer transformação $A$ é sempre 0 . De fato, $A(0)=A(0+0)=$ $A(0)+A(0)$, que pela lei do corte, implica $A(0)=0$. Além disso, dados $u, v \in E$ e $\alpha, \beta \in \mathbb{R}$, tem-se pelas propriedades acima $A(\alpha u+\beta v)=A(\alpha u)+A(\beta v)=$ $\alpha \cdot A(u)+\beta \cdot A(v)$. Como consequências imediatas, temos $A(-v)=-A(v) \mathrm{e}$ $A(u-v)=A(u)-A(v)$.

Quando a transformação linear $A: E \rightarrow F$ é bijetiva, dizemos que $A$ é um isomorfismo e que os conjuntos $E$ e $F$ são isomorfos. 
Uma transformação afim (ou mapeamento afim) $T$ entre dois espaços vetoriais consiste em uma transformação linear $A$ seguida por uma translação. Assim, se $b \in F$, podemos escrever

$$
\begin{aligned}
T: E & \rightarrow F \\
v & \mapsto A v+b
\end{aligned}
$$

Exemplo 2.4 Sejam duas transformações lineares $A, B: E \rightarrow F$. A soma de $A$ e $B$ é a transformação linear $A+B: E \rightarrow F$ definida por $(A+B) v=A v+B v$ para todo $v$. $O$ produto da transformação $A$ por um número $\alpha \in \mathbb{R}$ é a transformação linear $\alpha A: E \rightarrow F$ definida por $(\alpha A) v=\alpha \cdot$ Av para todo $v$. $O$ símbolo 0 indica a transformação linear nula $0: E \rightarrow F$ definida por $0(v)=0$ para todo $v$. A transformação $-A: E \rightarrow F$ é definida por $(-A) v=-A v$ para todo $v$. Desta forma, verifica-se que $(-A)+A=A+(-A)=0$.

Seja $\mathcal{L}(E ; F)$ o conjunto das transformações lineares de $E$ em $F$. As operações e funções definidas acima fazem de $\mathcal{L}(E ; F)$ um espaço vetorial. Em particular, as transformações lineares $\varphi: E \rightarrow \mathbb{R}$ são chamadas de funcionais lineares e o conjunto $\mathcal{L}(E ; \mathbb{R})$ é denotado por $E^{*}$ e chama-se o espaço vetorial dual de $E$.

Um subespaço vetorial de $X$ é um subconjunto $S \subset X$ com as seguintes propriedades:

(i) $0 \in S$;

(ii) Se $u, v \in S$ então $u+v \in S$;

(iii) Se $v \in S$ então, para todo $\alpha \in \mathbb{R}, \alpha v \in S$.

Exemplo 2.5 Evidentemente o conjunto $\{0\}$ e o espaço inteiro $X$ são exemplos triviais de subespaços de $X$. Todo subespaço é, em si mesmo, um espaço vetorial.

Exemplo 2.6 Qualquer reta que passa pela origem é um subespaço vetorial do plano.

Exemplo 2.7 Qualquer plano que passa pela origem é um subespaço de $\mathbb{R}^{3}$.

Dizemos que o subespaço vetorial de $X$ gerado por $S$ é, por definição, o conjunto de todas as combinações lineares de vetores $v_{i} \in S$ com $i \in\{1, \ldots, m\}$ denotado por $\operatorname{span} S$, isto é,

$$
\operatorname{span} S=\left\{\sum_{i=1}^{m} \alpha_{i} v_{i}: \alpha_{i} \in \mathbb{R}, v_{i} \in S\right\}
$$


Definição 2.10 Um conjunto $Y \subset X$ é chamado linearmente independente (ou L.I.) quando $\sum_{i=1}^{m} \alpha_{i} v_{i}=0$ com $v_{1}, v_{2}, \ldots, v_{m} \in Y$ se, e somente se, $\alpha_{1}=\alpha_{2}=\ldots=\alpha_{m}=0$.

Definição 2.11 Uma base de um espaço vetorial $X$ é um conjunto $\mathcal{B} \subset X$ linearmente independente que gera $X$.

Isto quer dizer que todo vetor $v \in X$ pode ser escrito de modo único como combinação linear $v=\sum_{i=1}^{m} \alpha_{i} v_{i}=0$ de elementos $v_{1}, v_{2}, \ldots, v_{m}$ da base $\mathcal{B}$. Os números $\alpha_{1}, \alpha_{2}, \ldots, \alpha_{m}$ são chamados coordenadas do vetor $v$ na base $\mathcal{B}$.

Dizemos que um espaço vetorial $X$ tem dimensão finita quando admite uma base $\mathcal{B}$ com um número finito $n$ de elementos. Este número chama-se a dimensão do espaço vetorial $X$.

Exemplo 2.8 Sejam $e_{1}=(1,0)$ e $e_{2}=(0,1)$. É fácil verificar que o conjunto $\left\{e_{1}, e_{2}\right\}$ é uma base de $\mathbb{R}^{2}$, chamada base canônica de $\mathbb{R}^{2}$. Portanto, o plano é um espaço vetorial de dimensão 2.

Exemplo 2.9 Mais geralmente, se $\mathcal{B}=\left\{v_{1}, v_{2}, \ldots, v_{m}\right\}$ é uma base do espaço vetorial $X$, então um funcional linear é totalmente determinado por suas imagens em cada $v_{i}$ e, por outro lado, valores arbitrários para cada $v_{i}$ determinam um funcional linear sobre $X$. De fato, os funcionais lineares $v_{i}^{*}$ com $(i=1,2, \ldots, m)$ definidos por

$$
v_{i}^{*}\left(v_{j}\right)=\delta_{i j} \quad(i, j=1, \ldots, m)
$$

tal que $\delta_{i j}=\left\{\begin{array}{ll}1 & \text { se } i=j \\ 0 & \text { se } i \neq j\end{array}\right.$ são linearmente independentes e geram $X^{*}$ porque $f\left(v_{j}\right)=\phi_{j}$ se, e somente se, $f=\sum_{i=1}^{m} \phi_{i} v_{i}^{*}$. Portanto, o conjunto $\mathcal{B}^{*}=\left\{v_{1}^{*}, \ldots, v_{m}^{*}\right\}$ é uma base de $X^{*}$ e é chamada base dual de $\mathcal{B}$. Isto é, $X$ e $X^{*}$ são espaços vetoriais de mesma dimensão.

Um produto interno em um espaço vetorial $X$ é uma função

$$
\begin{aligned}
\langle., .\rangle: X \times X & \rightarrow \mathbb{R} \\
(u, v) & \mapsto\langle u, v\rangle
\end{aligned}
$$

que satisfaz as seguintes propriedades para quaisquer $u, u^{\prime}, v, v^{\prime} \in X$ e $\alpha \in \mathbb{R}$ :

Bilinearidade: $\left\langle u+u^{\prime}, v\right\rangle=\langle u, v\rangle+\left\langle u^{\prime}, v\right\rangle,\langle\alpha u, v\rangle=\alpha\langle u, v\rangle,\left\langle u, v+v^{\prime}\right\rangle=$ $\langle u, v\rangle+\left\langle u, v^{\prime}\right\rangle,\langle u, \alpha v\rangle=\alpha\langle u, v\rangle$ 
Comutatividade (ou simetria): $\langle u, v\rangle=\langle v, u\rangle$;

Positividade: $\langle u, u\rangle>0$ se $u \neq 0$.

Seguem abaixo algumas consequências imediatas da definição acima:

1. $\langle 0, v\rangle=\langle v, 0\rangle=0$ para todo $v \in X$.

De fato, $\langle 0, v\rangle=\langle 0+0, v\rangle=\langle 0, v\rangle+\langle 0, v\rangle$ e, pela lei do corte, temos $\langle 0, v\rangle=0$.

2. $\langle u, v\rangle=0$ para todo $v \in X$ então $u=0$.

De fato, se fosse possível $u \neq 0$, pela positividade do produto interno teríamos $\langle u, v\rangle \neq 0$ pelo menos quando $v=u$, o que é absurdo.

3. Se $u, u^{\prime} \in X$ são vetores tais que $\langle u, v\rangle=\left\langle u^{\prime}, v\right\rangle$ para todo $v \in X$ então $u=u^{\prime}$.

De fato, temos que $\langle u, v\rangle-\left\langle u^{\prime}, v\right\rangle=0$, pela bilinearidade, segue que $\left\langle u-u^{\prime}, v\right\rangle=0$ para todo $v \in X$, logo, pela consequência $2, u-u^{\prime}=0 \Rightarrow$ $u=u^{\prime}$.

Exemplo 2.10 Sejam dois vetores $u$ e $v$ do espaço $\mathbb{R}^{n}$. Se $u=\left(u_{1}, u_{2}, \ldots, u_{n}\right) e$ $v=\left(v_{1}, v_{2}, \ldots, v_{n}\right)$ podemos definir o seguinte produto interno chamado produto interno canônico:

$$
\langle u, v\rangle=\sum_{i=1}^{n} u_{i} v_{i}
$$

Exemplo 2.11 Seja um vetor $x \in X$ com coordenadas $\left(\alpha_{1}, \ldots, \alpha_{n}\right)$ relativas a uma base $\mathcal{B}=\left\{\beta_{1}, \ldots, \beta_{n}\right\}$ e seja $f \in X^{*}$ com coordenadas $\left(\phi_{1}, \ldots, \phi_{n}\right)$ relativas à base dual $\mathcal{B}^{*}=\left\{\beta_{1}^{*}, \ldots, \beta_{n}^{*}\right\}$, podemos escrever

$$
f(x)=\phi_{1} \alpha_{1}+\ldots+\phi_{n} \alpha_{n}
$$

Seja $y=\left(\phi_{1}, \ldots, \phi_{n}\right) \in X$ na base $\mathcal{B}$. Podemos identificar y com o funcional linear $f_{y}=\left(\phi_{1}, \ldots, \phi_{n}\right)=\sum_{i=1}^{n} \phi_{i} \beta_{i}^{*}$ (escrito na base $\mathcal{B}^{*}$ ). Assim, se usarmos o produto interno canônico, podemos ainda escrever

$$
f_{y}(x)=\phi_{1} \alpha_{1}+\ldots+\phi_{n} \alpha_{n}=\langle y, x\rangle
$$


De acordo com Lima [8], no espaço $\mathbb{R}^{n}$ sempre podemos definir um produto interno mais geral da seguinte forma: toma-se uma matriz real $\left(a_{i j}\right)$, $n \times n$, simétrica, positiva (isto é, $\sum a_{i j} u_{i} u_{j}>0$ para todo $u \neq 0$ ), e põe-se

$$
\langle u, v\rangle=\sum_{i, j=1}^{n} a_{i j} u_{i} v_{j}
$$

É imediato verificar que a fórmula 2-3 satisfaz as condições de produto interno. Na verdade, o produto interno canônico (exemplo 2.10) é obtido a partir da definição acima quando tomamos a matriz identidade.

Portanto, qualquer espaço vetorial X de dimensão finita pode ser munido de um produto interno, pois basta, por exemplo, tomarmos o produto interno canônico neste espaço. Logo, podemos definir a noção de ortogonalidade entre dois vetores em qualquer espaço de dimensão finita, conforme Lima [7].

Definição 2.12 Seja X um espaço vetorial de dimensão finita. Dois vetores $u, v \in X$ chamam-se ortogonais (ou perpendiculares) quando $\langle u, v\rangle=0$. Neste caso, usamos a notação $u \perp v$.

Note que, em particular, 0 é ortogonal a qualquer vetor de $X$. Um conjunto $S \subset X$ é dito ortogonal quando quaisquer dois vetores distintos de $S$ são ortogonais.

Agora abordaremos o conceito de norma, que é fundamental para a definição da geometria de Minkowski. O resumo abaixo foi baseado em Thompson $[1]$.

Definição 2.13 Uma norma sobre um espaço vetorial $X$ é uma função

$$
\begin{aligned}
\|\cdot\|: \quad X & \rightarrow \mathbb{R}^{+} \\
x & \mapsto\|x\|
\end{aligned}
$$

que satisfaz os seguintes axiomas:

(i) $\|x\|=0$ se, e somente se, $x=0$;

(ii) $\|\alpha x\|=|\alpha|\|x\|$, com $\alpha \in \mathbb{R}$;

(iii) $\|x+y\| \leq\|x\|+\|y\|$ (desigualdade triangular).

Exemplo 2.12 Dado $u=\left(u_{1}, u_{2}\right) \in \mathbb{R}^{2}$, é trivial verificar que $\|u\|=\sqrt{\langle u, u\rangle}$ é uma norma do plano. Se tomarmos o produto interno canônico, então $\|u\|=\sqrt{u_{1}^{2}+u_{2}^{2}}$ é a norma euclideana do vetor $u$. 
Exemplo 2.13 Seja $X$ um espaço vetorial cuja base é $\left\{\beta_{1}, \ldots, \beta_{n}\right\}$. Dado $p \geq 1$ e $u \in X$, podemos escrever $u=\sum_{i=1}^{n} u_{i} \beta_{i}$ e definir uma norma chamada norma $\ell_{p}:$

$$
\|u\|_{p}=\left(\sum_{i=1}^{n}\left|u_{i}\right|^{p}\right)^{1 / p}
$$

A norma $\ell_{2}$ é a norma euclideana definida no exemplo 2.12, mas aqui definida mais geralmente para qualquer espaço de dimensão finita (em qualquer base). A norma $\ell_{1}$ é também conhecida como a norma da soma:

$$
\|u\|_{1}=\sum_{i=1}^{n}\left|u_{i}\right|
$$

Quando $p=\infty$, definimos a norma $\ell_{\infty}$ conhecida como norma do máximo conforme segue:

$$
\|u\|_{\infty}=\max \left\{\left|u_{i}\right| ; i=1, \ldots, n\right\}
$$

A verificação de que a norma $\ell_{p}$ é, de fato, uma norma pode ser encontrada em Thompson [1].

Exemplo 2.14 Seja $X$ um espaço vetorial. Seja $f: X \rightarrow \mathbb{R}$ um funcional linear. Podemos definir a seguinte norma sobre o espaço dos funcionais lineares limitados:

$$
\|f\|=\sup \{|f(x)| ;\|x\| \leq 1\}
$$

Esta norma será importante no capítulo 4 para definirmos o conceito de bola dual, que possui um importante papel na resposta do problema isoperimétrico em contextos mais gerais.

Definição 2.14 Uma métrica em um conjunto $X$ é uma função

$$
\begin{aligned}
d: X \times X & \rightarrow \mathbb{R}^{+} \\
(x, y) & \mapsto d(x, y)
\end{aligned}
$$

que satisfaz as seguintes condições para todo $x, y, z \in X$ :

(i) $d(x, x)=0$;

(ii) Se $x \neq y$ então $d(x, y) \neq 0$;

(iii) $d(x, y)=d(y, x)$;

(iv) $d(x, z) \leq d(x, y)+d(y, z)$. 
Assim, a partir da norma, encontramos uma forma natural de medir distâncias em qualquer espaço vetorial normado. Basta definirmos a métrica $d(x, y)=\|x-y\|$ e facilmente verificamos que $d$ é, de fato, uma métrica, pois, para todo $x, y, z \in X$ :

(i) $d(x, x)=\|x-x\|=\|0\|=0, \operatorname{logo} d(x, x)=0$;

(ii) Se $x \neq y$, então $d(x, y)=\|x-y\|$, como $x-y \neq 0, d(x, y) \neq 0$;

(iii) $d(x, y)=\|x-y\|=\|(-1)(y-x)\|=|-1|\|y-x\|=\|y-x\|=d(y, x)$;

(iv) $d(x, z)=\|x-z\|=\|(x-y)+(y-z)\| \leq\|x-y\|+\|y-z\|=d(x, y)+d(y, z)$.

Esta métrica gera uma topologia sobre $X$ chamada topologia induzida pela norma. Um espaço vetorial munido de uma norma é chamado espaço vetorial normado e quando ele tem dimensão finita é chamado espaço de Minkowski, conforme veremos no capítulo 4.

De acordo com Lima [8], duas normas arbitrárias $\|$.$\| e \|.\|^{*}$ sobre um espaço vetorial de dimensão finita $X$ são ditas equivalentes quando existirem constantes $a>0$ e $b>0$ tais que:

$$
a\|x\|^{*} \leq\|x\| \leq b\|x\|^{*}, \forall x \in X .
$$

A equivalência entre normas é uma relação reflexiva, simétrica e transitiva. Este conceito é fundamental por muitas razões, uma delas é que normas equivalentes dão origem à mesma noção de limite no espaço $X$. Outra razão é que se duas normas em $X$ são equivalentes, um conjunto $Y \subset X$ é limitado em relação a uma delas se, e somente se, é limitado em relação à outra. Também será importante para o conceito de continuidade que será abordado na seção 2.3, pois graças à equivalência entre normas o fato de uma aplicação ser contínua independe das normas escolhidas para o domínio ou contradomínio. Enfim, o teorema a seguir é de suma importância. Ainda segundo Lima [8]:

Teorema 2.1 Duas normas quaisquer no espaço vetorial de dimensão finita $X$ são equivalentes.

Prova. Vamos provar que uma norma arbitrária $\|$.$\| é equivalente à norma$ $\ell_{1}$ (a norma da soma). Por transitividade o teorema estará demonstrado. Digamos que a base de $X$ seja $\left\{\beta_{1}, \ldots, \beta_{n}\right\}$. Se $x \in X$, então podemos escrever $\|x\|_{1}=\sum_{i=1}^{n}\left|x_{i}\right|$. Tomemos $b=\max \left\{\left\|\beta_{1}\right\|, \ldots,\left\|\beta_{n}\right\|\right\}$. Então, para qualquer $x \in X$ temos

$$
\|x\|=\left\|\sum_{i=1}^{n} x_{i} \beta_{i}\right\| \leq \sum_{i=1}^{n}\left|x_{i}\right|\left\|\beta_{i}\right\| \leq b \sum_{i=1}^{n}\left|x_{i}\right|=b \cdot\|x\|_{1}
$$


Falta provar que existe $a>0$ tal que $a \cdot\|x\|_{1} \leq\|x\|$ para todo $x \in X$. Vamos supor, por absurdo, que isto seja falso. Então, para cada $k \in \mathbb{N}$, existe $x_{k} \in X$ tal que $k^{-1}\left\|x_{k}\right\|_{1}>\left\|x_{k}\right\|$. Seja $u_{k}=x_{k} /\left\|x_{k}\right\|_{1}$ e temos $\left\|u_{k}\right\|=\left\|x_{k}\right\| /\left\|x_{k}\right\|_{1}<k^{-1}$ e $\left\|u_{k}\right\|_{1}=1$ para todo $k$. Logo, a sequência $\left(u_{k}\right)$ é limitada com relação à norma da soma. Pelo Teorema de Bolzano-Weierstrass, ela possui uma subsequência $\left(u_{k_{j}}\right)$ que converge para um ponto $u \in X$. Isto quer dizer que $\|u\|_{1}=\lim _{j \rightarrow \infty}\left\|u_{k_{j}}\right\|_{1}=1$, donde $u \neq 0$. Mas pela desigualdade triangular e por $2-9$, para todo $j \in \mathbb{N}$ temos

$$
\|u\| \leq\left\|u_{k_{j}}-u\right\|+\left\|u_{k_{j}}\right\| \leq b\left\|u_{k_{j}}-u\right\|_{1}+k_{j}^{-1}
$$

Quando $j$ tende a infinito, as duas últimas parcelas acima tendem para zero. Portanto, $\|u\|=0$, donde $u=0$ pela definição de norma. Com esta contradição completamos a demonstração.

Estabelecida a noção de norma em espaços vetoriais de dimensões finitas, podemos definir segundo Lima [7] um tipo especial de elemento destes espaços:

Definição 2.15 Um vetor $\hat{u} \in X$ é um vetor unitário quando $\|\hat{u}\|=1$.

Todo vetor não-nulo $v$ pode ser escrito como $v=\|v\| \hat{v}$, em que $\hat{v}$ é o vetor unitário na mesma direção e sentido de $v$. Para isto, evidentemente basta tomar $\hat{v}=\frac{v}{\|v\|}$.

Ainda segundo Lima [7], temos:

Definição 2.16 Seja $X$ um espaço vetorial de dimensão finita. Se $\hat{u}$ é um vetor unitário e v é um vetor qualquer de $X$, o vetor $\langle v, \hat{u}\rangle \hat{u}$ é a projeção ortogonal de $v$ sobre a reta que contém $\hat{u}$.

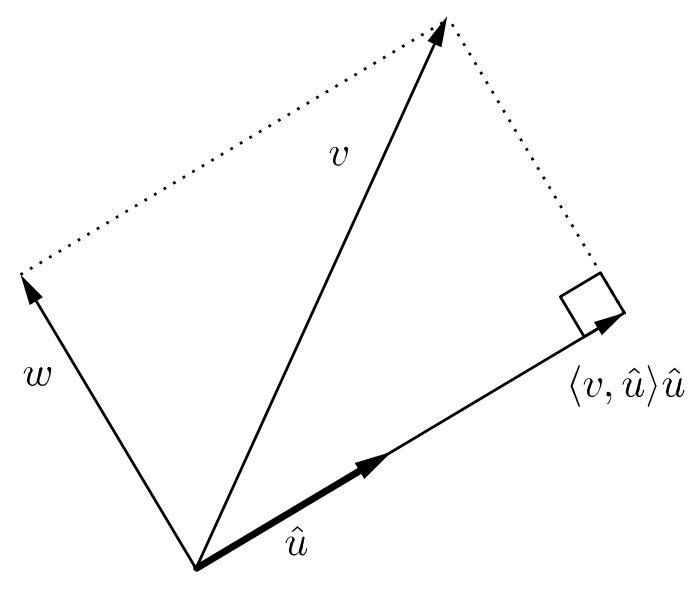

Figura 2.1: Projeção ortogonal de $v$ sobre a reta que contém $\hat{u}$. 
A definição acima é motivada pelo fato de que se tomarmos $w=$ $v-\langle v, \hat{u}\rangle \hat{u}$, então $w \perp u$ conforme mostra a figura 2.1. Com efeito, $\langle w, \hat{u}\rangle=$ $\langle v-\langle v, \hat{u}\rangle \hat{u}, \hat{u}\rangle=\langle v, \hat{u}\rangle-\langle v, \hat{u}\rangle\langle\hat{u}, \hat{u}\rangle=\langle v, \hat{u}\rangle-\langle v, \hat{u}\rangle=0$, pois $\langle\hat{u}, \hat{u}\rangle=1$.

\section{3}

\section{Aplicações contínuas}

As ideias a seguir são baseadas em Lima [8].

Sejam $E$ e $F$ espaços vetoriais normados de dimensões finitas. Sejam $\|\cdot\|_{E}$ e $\|\cdot\|_{F}$ as respectivas normas sobre $E$ e $F$. Seja $f: X \rightarrow F$ uma aplicação definida no conjunto $X \subset E$.

Definição 2.17 Dizemos que $f$ é contínua no ponto $a \in X$ quando, para qualquer $\varepsilon>0$ dado, podemos obter $\delta>0$ tal que se todo ponto $x \in X$ que satisfaz $\|x-a\|_{E}<\delta$, então $\|f(x)-f(a)\|_{F}<\varepsilon$.

Quando $f$ é contínua em todos os pontos do conjunto $X$, dizemos que $f$ é uma aplicação contínua. Uma aplicação $f: X \rightarrow F$ é chamada de Lipschitziana quando existe $k>0$ tal que, para quaisquer $x, y \in X$, temos $\|f(x)-f(y)\|_{F} \leq k\|x-y\|_{E}$. O número $k$ é chamado de constante de Lipschitz de $f$.

Teorema 2.2 Toda aplicação Lipschitziana é contínua.

Prova. Dado $\varepsilon>0$, basta tomarmos $\delta=\frac{\varepsilon}{k}$. Então, se $a \in X,\|x-a\|_{E}<\delta \Rightarrow$ $\|f(x)-f(a)\|_{F} \leq k\|x-a\|_{E}<k \cdot \frac{\varepsilon}{k}=\varepsilon$. Como isto é válido para todos pontos $a \in X$, segue que $f$ é contínua.

Apesar de termos denotado normas distintas para os espaços $E$ e $F$, a equivalência das normas permite que o teorema 2.2 seja válido independentemente das normas escolhidas.

Teorema 2.3 Toda transformação linear $A: E \rightarrow F$ é Lipschitziana e, portanto, contínua.

Prova. Sejam $m$ e $n$ as respectivas dimensões de $E$ e $F$. Seja $\left\{u_{1}, \ldots, u_{m}\right\}$ a base de $E$. Vamos considerar agora $\|\cdot\|_{E}=\|\cdot\|_{1}$, isto é, a norma sobre $E$ será a norma $\ell_{1}$ (a norma da soma definida em 2-5). Tome $c=$ $\max \left\{\left\|A\left(u_{1}\right)\right\|_{F}, \ldots,\left\|A\left(u_{m}\right)\right\|_{F}\right\}$. Então, para todo $x=\sum_{i=1}^{m} x_{i} u_{i}$ temos:

$\|A(x)\|_{F}=\left\|A\left(\sum_{i=1}^{m} x_{i} u_{i}\right)\right\|_{F}=\left\|\sum_{i=1}^{m} x_{i} A\left(u_{i}\right)\right\|_{F} \leq \sum_{i=1}^{m}\left|x_{i}\right| \cdot\left\|A\left(u_{i}\right)\right\|_{F} \leq\left[\sum_{i=1}^{m}\left|x_{i}\right|\right] \cdot c$ 
Como $\sum_{i=1}^{m}\left|x_{i}\right|=\|x\|_{1}$, temos $\|A(x)\|_{F} \leq c\|x\|_{1}$ para todo $x \in E$. Logo, para $x, y \in E$ arbitrários, vale:

$$
\|A(x)-A(y)\|_{F}=\|A(x-y)\|_{F} \leq c\|x-y\|_{1}
$$

Na verdade, mostramos que a aplicação $A$ é Lipschitziana com relação à norma $\ell_{1}$ para o seu domínio $E$, mas o teorema 2.1 garante que quaisquer duas normas em espaços de dimensão finita são equivalentes, $\log$ o $A$ é Lipschitziana com relação à qualquer norma e, consequentemente pelo teorema 2.2 , é contínua.

\section{4}

\section{Curvas planas}

Os conceitos expostos a seguir são baseados em Alencar \& Santos [3].

Nesta seção, vamos considerar $I \subset \mathbb{R}$ um intervalo e uma aplicação $\alpha: I \rightarrow \mathbb{R}^{2}$ dada por $\alpha(s)=(x(s), y(s))$.

Definição 2.18 A aplicação a é uma curva contínua no plano $\mathbb{R}^{2}$ quando é contínua.

O conjunto imagem $c$ da aplicação $\alpha$ dado por:

$$
c=\{\alpha(s) ; s \in I\}
$$

é chamado traço de $\alpha$.

Se a curva $\alpha$ está definida em um intervalo fechado $I=[a, b]$, os pontos $\alpha(a)$ e $\alpha(b)$ são chamados ponto inicial e ponto final de $\alpha$, respectivamente. Quando $\alpha(a)=\alpha(b)$, dizemos que $\alpha$ é uma curva fechada.

Se $I=\mathbb{R}$, então a aplicação $\alpha$ é dita periódica quando existe um número real $l>0$, tal que $\alpha(s+l)=\alpha(s)$ para todo $s \in \mathbb{R}$. O número $l$ é chamado período de $\alpha$. O menor valor $l_{0}$ para o qual esta equação se verifica é chamado período fundamental de $\alpha$.

Se $I$ é um intervalo qualquer, uma curva $\alpha$ é dita simples, quando a aplicação $\alpha$ é injetiva. Isto é, se $\alpha\left(s_{1}\right)=\alpha\left(s_{2}\right)$, então $s_{1}=s_{2}$. Se $I=[a, b]$, uma curva $\alpha$ é dita fechada e simples (também denominada curva de Jordan) quando $\alpha$ restrita ao intervalo $[a, b)$ for injetiva e $\alpha(a)=\alpha(b)$, isto é, quando os únicos pontos $\alpha\left(s_{1}\right)$ e $\alpha\left(s_{2}\right)$ tais que $\alpha\left(s_{1}\right)=\alpha\left(s_{2}\right)$ com $s_{1} \neq s_{2}$ são os pontos inicial e final.

Voltando a considerar $I$ um intervalo qualquer. Temos a seguinte definição. 
Definição 2.19 Uma aplicação $\alpha$ é uma curva parametrizada suave ou um caminho no plano $\mathbb{R}^{2}$ quando cada função coordenada $x, y: I \rightarrow \mathbb{R}$ é uma função de classe $\mathcal{C}^{\infty}$, isto é, $x$ e y possuem derivadas contínuas de qualquer ordem em todo ponto de I.

O vetor tangente (ou vetor velocidade) de $\alpha$ em $s_{0} \in I$ é dado por:

$$
\alpha^{\prime}\left(s_{0}\right)=\left(x^{\prime}\left(s_{0}\right), y^{\prime}\left(s_{0}\right)\right)
$$

Definição 2.20 Dizemos que uma curva parametrizada $\alpha$ é regular em $s_{0} \in I$, quando $\alpha^{\prime}\left(s_{0}\right) \neq(0,0)$, ou equivalentemente, quando $\left\|\alpha^{\prime}\left(s_{0}\right)\right\| \neq 0$. A curva $\alpha$ é regular em $I$, quando $\alpha$ for regular para todo $t \in I$. Quando $\left\|\alpha^{\prime}\left(s_{0}\right)\right\|=0$, dizemos que $\alpha$ é singular em $s_{0}$ e $\alpha\left(s_{0}\right)$ é chamada uma singularidade de $\alpha$.

A função $L: I \rightarrow \mathbb{R}$, definida por:

$$
L(s)=\int_{s_{0}}^{s}\left\|\alpha^{\prime}(\xi)\right\| d \xi
$$

$s_{0} \in I$, é denominada comprimento de arco.

Se $I=[a, b]$, então o comprimento de $\alpha$ é dado por $L=L(b)-L(a)$.

Definição 2.21 Uma curva a está parametrizada pelo comprimento de arco quando:

$$
\left\|\alpha^{\prime}(s)\right\| \equiv 1
$$

É possível verificar que toda curva regular pode ser parametrizada pelo comprimento de arco. [3] Note que, se $\alpha$ está parametrizada por comprimento de arco, então está bem definido um campo $t$ de vetores tangentes e unitários ao longo de $\alpha$ dado por $t(s)=\alpha^{\prime}(s)$. Isto é, $t(s)$ é o vetor tangente à curva $\alpha$ em $\alpha(s)$ e temos $t(s)=\left(x^{\prime}(s), y^{\prime}(s)\right)$. Desta forma, podemos definir o campo $n$ ao longo de $\alpha$, tal que, para cada $s \in I,\{t, n\}$ seja uma base positiva de $\mathbb{R}^{2}$, isto é, existe uma rotação que leva $(1,0)$ em $t$ e $(0,1)$ em $n$. Logo, $n(s)=\left(-y^{\prime}(s), x^{\prime}(s)\right)$ e é claro que $n$ é um campo normal e unitário ao longo de $\alpha$ de classe $\mathcal{C}^{\infty}$.

Definição 2.22 Para cada $s \in I, n(s)$ é chamado vetor normal à curva $\alpha$ em $\alpha(s)$.

Exemplo 2.15 A aplicação $c:[0,2 \pi R] \rightarrow \mathbb{R}^{2}$ dada por $c(t)=$ $R\left(\cos \left(\frac{t}{R}\right)\right.$, sen $\left.\left(\frac{t}{R}\right)\right)$ é uma parametrização por comprimento de arco do círculo de raio $R$ e centro na origem. O gráfico de c está na figura 2.2. 


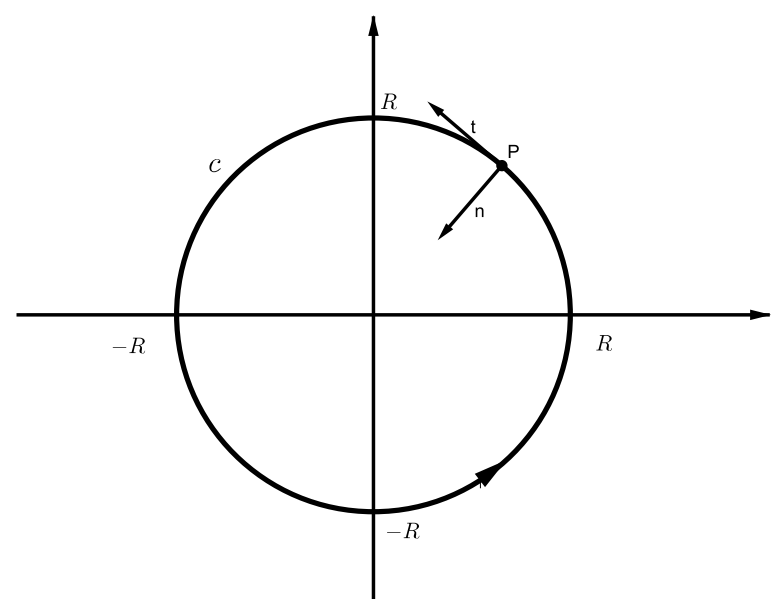

Figura 2.2: Círculo de raio $\mathrm{R}$ e centro na origem.

Se $\alpha$ é uma curva parametrizada pelo comprimento de arco, temos as fórmulas de Frenet abaixo:

$$
\frac{d \alpha}{d s}=t, \quad \frac{d t}{d s}=k n, \quad \frac{d n}{d s}=-k t
$$

Definição 2.23 O número real $k=k(s)$ que satisfaz a condição acima é chamado curvatura de $\alpha$ em $\alpha(s)$. Quando $k\left(s_{0}\right)>0$, definimos o raio de curvatura de $\alpha$ em $s_{0}$ por $\rho_{0}=\frac{1}{k\left(s_{0}\right)}$.

O ponto $P_{\rho_{0}}=\alpha\left(s_{0}\right)+\frac{1}{k\left(s_{0}\right)} N\left(s_{0}\right)$ é chamado centro de curvatura ou ponto focal de $\alpha$ em $s_{0}$ e o círculo $C_{\rho_{0}}$ é chamado círculo osculador de $\alpha$ em $s_{0}$.

\section{5}

\section{A função suporte}

A função suporte, que será definida a seguir, nos fornece fórmulas úteis para a determinação de comprimento de curvas planas e a área da região limitada por elas. Esta função também é importante na compreensão do conceito de área mista, que será fundamental neste trabalho. As ideias expostas a seguir são baseadas em Flanders [9].

Seja $c$ o traço de uma curva convexa, fechada e suave de $\mathbb{R}^{2}$. Sejam $\alpha=$ $\alpha(s)$ a parametrização por comprimento de arco desta curva, $t=t(s)$ o vetor tangente e $n=n(s)$ o vetor normal. Considere que $c$ possui curvatura positiva em todo ponto. Seja $L$ o comprimento de $c$, então $\alpha$ é uma curva periódica de período $L$. As funções $t, n, k$ também têm período (não necessariamente fundamental) $L$. 
Por 2-10, como $\left\|\alpha^{\prime}(s)\right\|=\|t(s)\| \equiv 1$, temos que:

$$
L=\oint_{c} d s
$$

Seja $d \alpha=(d x, d y)=t d s$, temos:

$$
\begin{gathered}
(-d y, d x)=n d s \\
\langle(x, y),(-d y, d x)\rangle=\langle\alpha, n d s\rangle \\
-(y d x-x d y)=-\langle\alpha, n d s\rangle
\end{gathered}
$$

Pelo teorema de Green, a área da região limitada pela curva $c$ é dada por $A=-\frac{1}{2} \oint_{c}(y d x-x d y), \log \mathrm{O}:$

$$
A=-\frac{1}{2} \oint_{c}\langle\alpha, n\rangle d s
$$

Por conveniência, considere a origem no interior da curva $c$. Ela será chamada de centro da curva $c$.

Definição 2.24 A função suporte $p: \mathbb{R} \rightarrow \mathbb{R}^{+}$é dada pela distância entre a origem e a reta tangente à curva c no ponto $\alpha(s)$ para $s \in[0, L)$. Ver figura 2.3.

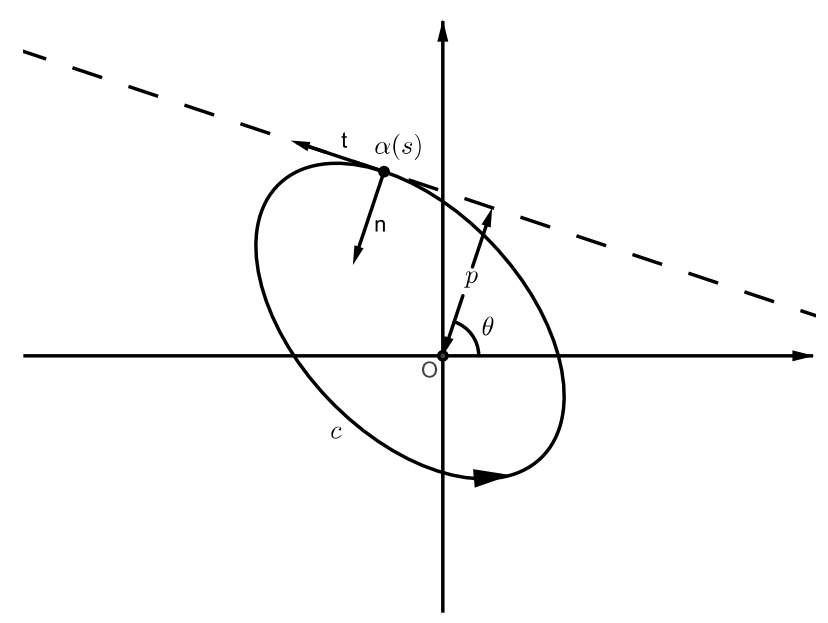

Figura 2.3: Função suporte.

Note que, pela convexidade de $c$, cada ponto $\alpha(s)$ da curva está associado a um único $\theta \in[0,2 \pi)$. O período de $p$ é $2 \pi$. Portanto, para cada $\theta$, podemos pensar em $p(\theta)$ como a norma do vetor projeção ortogonal de $\alpha(s)$ sobre a reta que contém o vetor unitário $-n(s)$, conforme definição 2.16. Isto é, $p(\theta)=\|\langle\alpha(s),-n(s)\rangle \cdot(-n(s))\|$ e temos:

$$
p(\theta)=-\langle\alpha(s), n(s)\rangle
$$


Pela definição de $\theta$, temos:

$$
n=(-\cos \theta,-\operatorname{sen} \theta)
$$

Com a rotação de $\pi / 2$ no sentido anti-horário do vetor $n$, obtemos:

$$
t=(-\operatorname{sen} \theta, \cos \theta)
$$

Assim, temos:

$$
\frac{d t}{d \theta}=n, \quad \frac{d n}{d \theta}=-t
$$

Logo, $d t=n d \theta$. Mas por $2-11, d t=k n d s$ e segue que:

$$
d \theta=k d s
$$

Agora derivando 2-14 e considerando $d / d \theta$ como ('), obtemos:

$$
p^{\prime}(\theta)=-\left\langle\alpha^{\prime}(\theta), n(\theta)\right\rangle-\left\langle\alpha(\theta), n^{\prime}(\theta)\right\rangle
$$

Por 2-17,

$$
p^{\prime}(\theta)=-\left\langle\alpha^{\prime}(\theta), n(\theta)\right\rangle+\langle\alpha(\theta), t(\theta)\rangle
$$

Derivando $\alpha(s)$ em relação a $\theta$ e usando 2-11, obtemos:

$$
\alpha^{\prime}(s)=\frac{d \alpha}{d s} \cdot s^{\prime}=s^{\prime} t
$$

Isto significa que $\alpha^{\prime}$ é paralelo a $t, \operatorname{logo} \alpha^{\prime}$ é perpendicular a $n$ e $\left\langle\alpha^{\prime}(\theta), n(\theta)\right\rangle=0$. Portanto,

$$
p^{\prime}(\theta)=\langle\alpha(\theta), t(\theta)\rangle
$$

Derivando novamente a equação 2-20, obtemos:

$$
p^{\prime \prime}(\theta)=\left\langle\alpha^{\prime}(\theta), t(\theta)\right\rangle+\left\langle\alpha(\theta), t^{\prime}(\theta)\right\rangle=\left\langle\alpha^{\prime}(\theta), t(\theta)\right\rangle+\langle\alpha(\theta), n(\theta)\rangle
$$

Como $\langle\alpha, n\rangle=-p$ e, por $2-19,\left\langle\alpha^{\prime}, t\right\rangle=\left\langle\left(s^{\prime} t\right), t\right\rangle=s^{\prime}\langle t, t\rangle=s^{\prime}$, segue que:

$$
p^{\prime \prime}=s^{\prime}-p
$$

Logo,

$$
s^{\prime}=p+p^{\prime \prime}
$$

Por 2-18, concluímos que $s^{\prime}=\frac{d s}{d \theta}=\frac{1}{k}=\rho$, isto é, conforme definido em 2.23, $\rho$ é o raio de curvatura de $c$ e podemos escrever a equação acima assim:

$$
\rho=p+p^{\prime \prime}
$$


Agora a integral 2-12 pode ser reescrita da seguinte forma:

$$
L=\oint d s=\int_{0}^{2 \pi} \rho d \theta=\int_{0}^{2 \pi}\left(p+p^{\prime \prime}\right) d \theta
$$

Como $p^{\prime \prime} d \theta=d\left(p^{\prime}\right)$ e $p^{\prime}$ tem período $2 \pi$, temos que $\oint p^{\prime \prime} d \theta=\oint d\left(p^{\prime}\right)=$ $\left.p^{\prime}(\theta)\right]_{0}^{2 \pi}=0$ e segue:

$$
L=\int_{0}^{2 \pi} p d \theta
$$

A integral 2-13 que representa a área também pode ser reescrita de outra forma. Por 2-14 e por 2-21, temos:

$$
A=-\frac{1}{2} \oint_{c}\langle\alpha, n\rangle d s=\frac{1}{2} \int_{0}^{2 \pi} p\left(p+p^{\prime \prime}\right) d \theta
$$

Como $d\left(p p^{\prime}\right)=p^{\prime} p^{\prime} d \theta+p p^{\prime \prime} d \theta, \int_{0}^{2 \pi} d\left(p p^{\prime}\right)=\int_{0}^{2 \pi}\left(p^{\prime}\right)^{2} d \theta+\int_{0}^{2 \pi} p p^{\prime \prime} d \theta$. Mas $\left.p p^{\prime}(\theta)\right]_{0}^{2 \pi}=0, \log 0 \int_{0}^{2 \pi} p p^{\prime \prime} d \theta=-\int_{0}^{2 \pi}\left(p^{\prime}\right)^{2} d \theta$ e finalmente obtemos:

$$
A=\frac{1}{2} \int_{0}^{2 \pi}\left[p^{2}-\left(p^{\prime}\right)^{2}\right] d \theta
$$

Podemos observar que a função suporte determina a curva $c$ de forma única. Isto é, pela definição 2.16, podemos escrever $\alpha=-\langle\alpha, n\rangle(-n)+\langle\alpha, t\rangle$ t. Então por 2-14 e por 2-20, temos que:

$$
\alpha=-p n+p^{\prime} t
$$

Logo, por 2-15 e 2-16, comprovamos nossa observação:

$$
\alpha(\theta)=\left(p \cos \theta-p^{\prime} \operatorname{sen} \theta, p \operatorname{sen} \theta+p^{\prime} \cos \theta\right)
$$

E ainda, conforme Craizer [10], se considerarmos $e_{r}=(\cos \theta, \operatorname{sen} \theta)$ e $e_{\theta}=(-\operatorname{sen} \theta, \cos \theta)$ podemos escrever a fórmula acima como

$$
\alpha(\theta)=p(\theta) e_{r}+p^{\prime}(\theta) e_{\theta}
$$

Podemos interpretar geometricamente que o conjunto convexo $K$, cuja fronteira é a curva $c$, é a interseção de todos os semi-planos determinados pelas retas tangentes à $c$ que contém $K$. Neste caso, dizemos que as tangentes à $c$ envelopam c conforme mostra a figura 2.4. Isto pode ser escrito da seguinte forma:

$$
K=\left\{v \in \mathbb{R}^{2} \mid-\langle v, n(\theta)\rangle \leq p(\theta), \forall \theta\right\}
$$




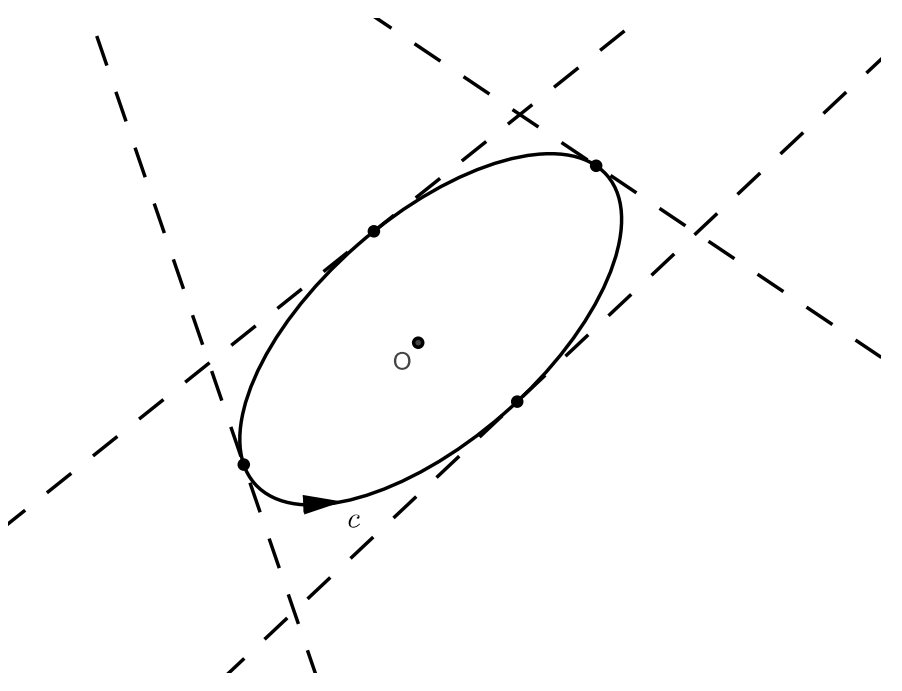

Figura 2.4: As tangentes à c envelopam $c$. 


\section{3}

\section{A desigualdade de Minkowski}

Neste capítulo demonstraremos a desigualdade de Minkowski. Ela será fundamental para a compreensão do problema isoperimétrico clássico da geometria euclideana plana e, mais geralmente, do problema isoperimétrico para uma geometria de Minkowski. Os conceitos e resultados a seguir são baseados em Flanders [9]. Em todo este capítulo, o perímetro considerado será euclideano e a área, a usual.

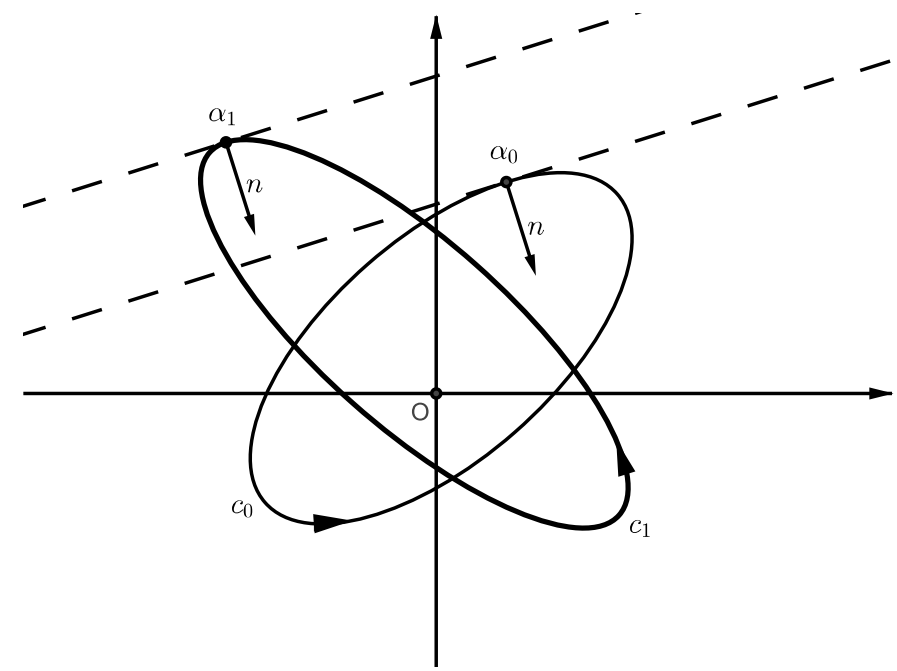

Figura 3.1: As curvas $c_{0}$ e $c_{1}$ têm a mesma normal nos pontos $\alpha_{0}$ e $\alpha_{1}$ respectivamente.

Sejam duas curvas convexas, fechadas e suaves $c_{0}$ e $c_{1}$ tais que as funções $\alpha_{0}=\alpha_{0}(\theta)$ e $\alpha_{1}=\alpha_{1}(\theta)$ representem os respectivos pontos destas curvas. Considere a correspondência biunívoca entre os pontos $\alpha_{0}$ e $\alpha_{1}$ que têm a mesma normal $n=n(\theta)$, conforme mostra a figura 3.1. Desta forma, as funções suporte são dadas por:

$$
p_{0}(\theta)=-\left\langle\alpha_{0}, n\right\rangle, \quad p_{1}(\theta)=-\left\langle\alpha_{1}, n\right\rangle
$$

Assim como na seção 2.5, vamos considerar a origem no interior das curvas $c_{0}$ e $c_{1}$ e ela será chamada de centro das curvas $c_{0}$ e $c_{1}$. 
Sejam $K_{0}$ e $K_{1}$ as regiões limitadas pelas curvas $c_{0}$ e $c_{1}$ respectivamente. A soma de Minkowski é o conjunto $K$ definido por

$$
K=K_{0}+K_{1}=\left\{v_{0}+v_{1} \mid v_{0} \in K_{0}, v_{1} \in K_{1}\right\}
$$

Seja $c$ a fronteira da nova região $K=K_{0}+K_{1}$. Geometricamente podemos construir $c$, transladando $c_{1}$ de maneira que seu centro seja um ponto $\alpha_{0}$ de $c_{0}$. Um ponto da curva $c$ é, conforme definido acima, o ponto $\alpha_{1}$ de $c_{1}$ associado ao ponto $\alpha_{0}$ após a translação. A curva $c$ resulta de todas estas possíveis translações. A figura 3.2 nos ajuda a entender que a função suporte $p$ da curva $c$ é dada por

$$
p=p_{0}+p_{1}
$$

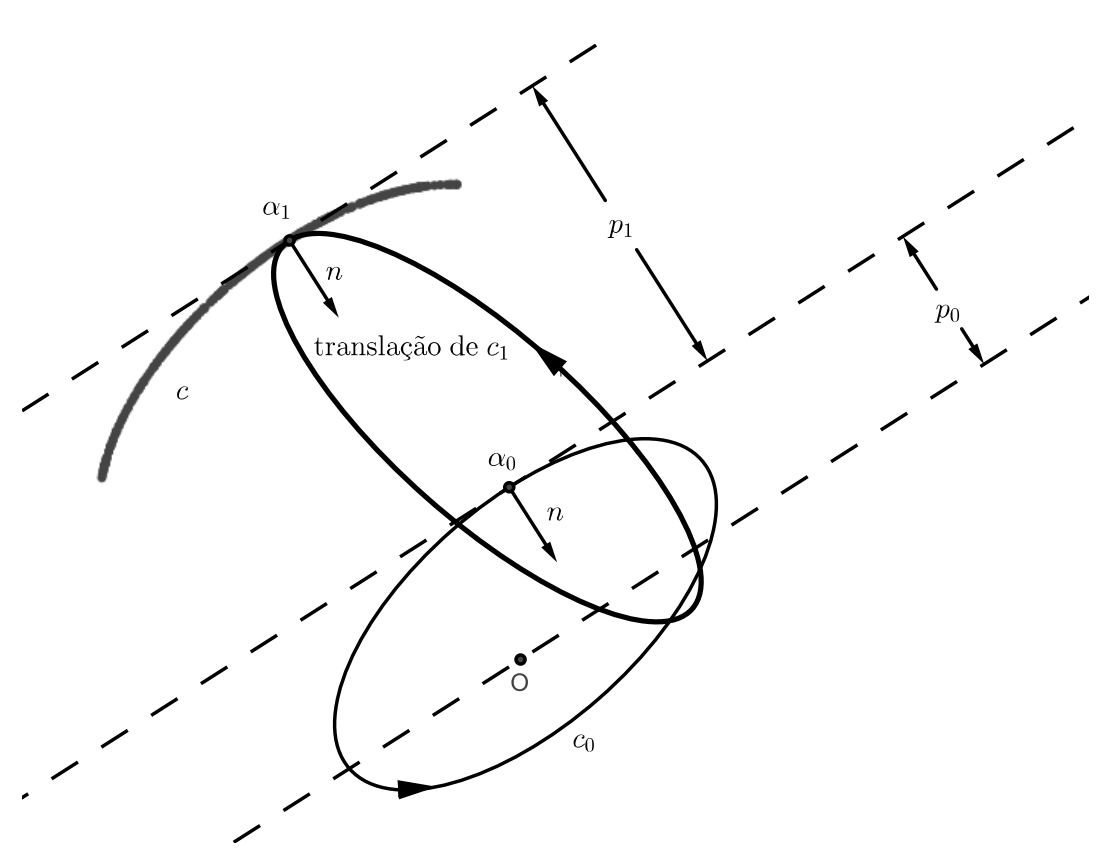

Figura 3.2: Translação da curva $c_{1}$ tal que o seu centro fique sobre a curva $c_{0}$.

De fato, se $v_{0} \in K_{0}$ e $v_{1} \in K_{1}$, pela definição 2-26 aplicada à $K_{0}$ e $K_{1}$, então

$$
-\left\langle\left(v_{0}+v_{1}\right), n(\theta)\right\rangle=-\left\langle v_{0}, n(\theta)\right\rangle-\left\langle v_{1}, n(\theta)\right\rangle \leq p_{0}(\theta)+p_{1}(\theta)
$$

Por outro lado, $\alpha_{0}(\theta) \in K_{0}$ e $\alpha_{1}(\theta) \in K_{1}$, logo $\alpha_{0}(\theta)+\alpha_{1}(\theta) \in K$ e temos

$$
-\left\langle\left[\alpha_{0}(\theta)+\alpha_{1}(\theta)\right], n(\theta)\right\rangle=-\left\langle\alpha_{0}(\theta), n(\theta)\right\rangle-\left\langle\alpha_{1}(\theta), n(\theta)\right\rangle=p_{0}(\theta)+p_{1}(\theta)
$$

Portanto, $K$ é a interseção de todos os semi-planos $\{v \mid-\langle v, n(\theta)\rangle \leq$ $\left.p_{0}(\theta)+p_{1}(\theta)\right\}$ e segue que $K$ é um conjunto convexo cuja fronteira $c$ tem função suporte $p=p_{0}+p_{1}$. 
Definição $3.1 A$ área mista de $c_{0}$ e $c_{1}$ é dada por

$$
A_{01}=\frac{1}{2} \int_{0}^{2 \pi}\left[p_{0} p_{1}-p_{0}^{\prime} p_{1}^{\prime}\right] d \theta
$$

Lema 3.1 Sejam $L_{i}$ o comprimento de $c_{i}(i=0,1), A_{i}$ a área da região limitada por $c_{i}$ e $A_{01}$ a área mista de $c_{0}$ e $c_{1}$. O comprimento de c e a área limitada por c são:

$$
L=L_{0}+L_{1}, \quad A=A_{0}+2 A_{01}+A_{1}
$$

Prova.

$$
\begin{aligned}
L & =\int_{0}^{2 \pi} p d \theta=\int_{0}^{2 \pi}\left(p_{0}+p_{1}\right) d \theta=\int_{0}^{2 \pi} p_{0} d \theta+\int_{0}^{2 \pi} p_{1} d \theta=L_{0}+L_{1} \\
A & =\frac{1}{2} \int_{0}^{2 \pi}\left(p^{2}-p^{\prime 2}\right) d \theta \\
& =\frac{1}{2} \int_{0}^{2 \pi}\left[\left(p_{0}^{2}+2 p_{0} p_{1}+p_{1}^{2}\right)-\left(p_{0}^{\prime 2}+2 p_{0}^{\prime} p_{1}^{\prime}+p_{1}^{\prime 2}\right)\right] d \theta \\
& =\frac{1}{2}\left\{\int_{0}^{2 \pi}\left(p_{0}^{2}-p_{0}^{\prime 2}\right) d \theta+2 \int_{0}^{2 \pi}\left(p_{0} p_{1}-p_{0}^{\prime} p_{1}^{\prime}\right) d \theta+\int_{0}^{2 \pi}\left(p_{1}^{2}-p_{1}^{\prime 2}\right) d \theta\right\} \\
& =A_{0}+2 A_{01}+A_{1}
\end{aligned}
$$

Observação: É trivial verificar que a área mista relativa a duas curvas iguais é a área da região delimitada pela curva. De fato, pela definição e pela fórmula 2-24, temos

$$
A_{00}=\frac{1}{2} \int_{0}^{2 \pi}\left[p_{0}^{2}-p_{0}^{\prime 2}\right] d \theta=A_{0}
$$

Podemos escrever a área mista de outra forma. Se $d\left(p_{0} p_{1}^{\prime}\right)=\left(p_{0}^{\prime} p_{1}^{\prime}+\right.$ $\left.p_{0} p_{1}^{\prime \prime}\right) d \theta$ e procedendo de forma análoga ao que foi feito na seção 2.5 para a dedução da fórmula 2-24 para área, obtemos $\oint p_{0}^{\prime} p_{1}^{\prime} d \theta=-\oint p_{0} p_{1}^{\prime \prime} d \theta$ e, então

$$
A_{01}=\frac{1}{2} \int_{0}^{2 \pi} p_{0}\left(p_{1}+p_{1}^{\prime \prime}\right) d \theta
$$

Por 2-21, temos:

$$
A_{01}=\frac{1}{2} \int_{0}^{2 \pi} p_{0} d s_{1}=A_{10}=\frac{1}{2} \int_{0}^{2 \pi} p_{1} d s_{0}
$$

Craizer [10] ainda determina uma outra fórmula para a área mista. Denotemos por $[u, v]$ o determinante de uma matriz $2 \times 2$ cujas colunas são os vetores $u$ e $v$ de $\mathbb{R}^{2}$. Se escrevermos $\alpha_{0}$ e $\alpha_{1}$ conforme a fórmula 2- 
25 da seção 2.5, temos $\alpha_{i}(\theta)=p_{i}(\theta) e_{r}+p_{i}^{\prime}(\theta) e_{\theta}$ para $i \in\{1,2\}$. Note que $\alpha_{1}^{\prime}(\theta)=p_{1}^{\prime}(\theta) e_{r}+p_{1}(\theta) e_{\theta}+p_{1}^{\prime \prime}(\theta) e_{\theta}-p_{1}^{\prime}(\theta) e_{r}$ e então $\alpha_{1}^{\prime}(\theta)=\left(p_{1}(\theta)+p_{1}^{\prime \prime}(\theta)\right) e_{\theta}$. Agora ao calcular o determinante $\left[\alpha_{0}, \alpha_{1}^{\prime}\right]$, vemos que

$$
\begin{aligned}
{\left[\alpha_{0}(\theta), \alpha_{1}^{\prime}(\theta)\right] } & =\left[p_{0} e_{r}+p_{0}^{\prime} e_{\theta},\left(p_{1}+p_{1}^{\prime \prime}\right) e_{\theta}\right] \\
& =\left(p_{1}+p_{1}^{\prime \prime}\right)\left(p_{0}\left[e_{r}, e_{\theta}\right]+p_{0}^{\prime}\left[e_{\theta}, e_{\theta}\right]\right) \\
& =\left(p_{1}+p_{1}^{\prime \prime}\right) p_{0}
\end{aligned}
$$

pois evidentemente $\left[e_{r}, e_{\theta}\right]=1 \mathrm{e}\left[e_{\theta}, e_{\theta}\right]=0$. Por $3-2$, finalmente obtemos

$$
A_{01}=\frac{1}{2} \int_{0}^{2 \pi}\left[\alpha_{0}, \alpha_{1}^{\prime}\right] d \theta
$$

Agora vamos transladar a curva $c_{1}$ de maneira que $c_{1}$ se torne tangente à curva $c_{0}$ externamente conforme mostra a figura 3.3. Assim, o lugar geométrico de todos os centros destas curvas transladadas será uma nova curva que chamaremos de $c_{2}$. A curva $c_{2}$ será convexa com função suporte $p_{2}(\theta)=$ $p_{0}(\theta)+p_{1}(\theta+\pi)$. Isto é, se $p_{1}(\theta+\pi)$ é a função suporte da curva $c_{1}^{*}$, que

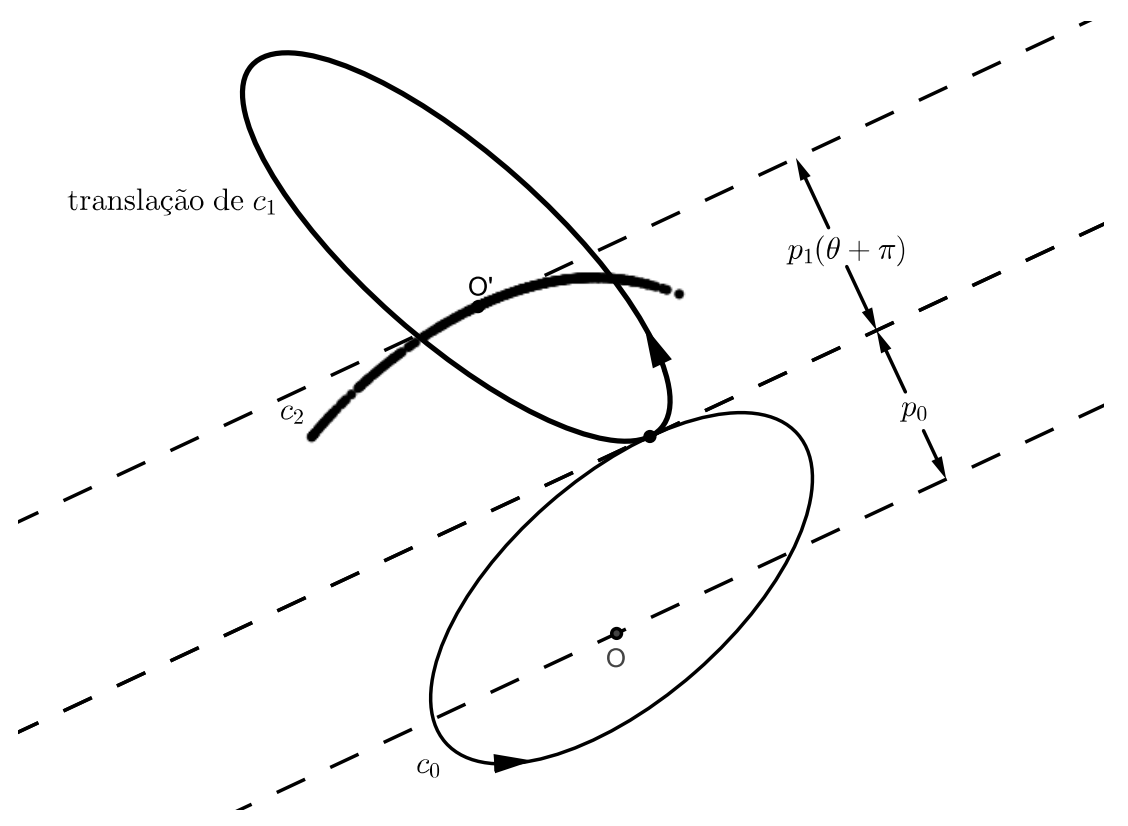

Figura 3.3: Translação da curva $c_{1}$ tal que ela tangencie a curva $c_{0}$. 
Lema 3.2 O comprimento de $c_{2}$ e a área limitada por $c_{2}$ são:

$$
L_{2}=L_{0}+L_{1}, \quad A_{2}=A_{0}+2 A_{01}^{*}+A_{1}
$$

em que

$$
\begin{aligned}
A_{01}^{*} & =\frac{1}{2} \int_{0}^{2 \pi}\left[p_{0}(\theta) p_{1}(\theta+\pi)-p_{0}^{\prime}(\theta) p_{1}^{\prime}(\theta+\pi)\right] d \theta \\
& =\frac{1}{2} \int_{0}^{2 \pi} p_{1}(\theta+\pi) d s_{0}(\theta)
\end{aligned}
$$

Prova. Evidentemente $c_{1}$ e $c_{1}^{*}$ têm mesmo comprimento e limitam regiões de mesma área, pois uma curva é apenas a rotação da outra. Logo, podemos aplicar o lema 3.1 para obtermos $L_{2}$ e $A_{2}$, sendo que basta considerar $p_{1}^{*}(\theta)=$ $p_{1}(\theta+\pi)$.

Antes de enunciarmos o teorema 3.1, precisamos de algumas definições básicas.

Definição 3.2 O raio inscrito de $c_{0}$ relativo a $c_{1}$ é o maior número real $r_{0}$ tal que a translação de $r_{0} K_{1}$ esteja contida em $K_{0}$.

Definição 3.3 O raio circunscrito de $c_{0}$ relativo a $c_{1}$ é o menor número real $R_{0}$ tal que a translação de $R_{0} K_{1}$ contém $K_{0}$.

Se $c_{1}$ é o círculo unitário, então dizemos que $r_{0}$ e $R_{0}$ são simplesmente o raio inscrito e o raio circunscrito de $c_{0}$ respectivamente. É claro que $R_{0} \geq r_{0}$, sendo $R_{0}=r_{0}$ se, e somente se, $K_{0}$ é uma translação de $r_{0} K_{1}$. A figura 3.4 nos ajuda a entender as definições acima.

Teorema 3.1 (Desigualdade de Minkowski) Sejam $c_{0}$ e $c_{1}$ curvas convexas fechadas que limitam regiões de áreas $A_{0}$ e $A_{1}$ respectivamente. Seja $A_{01}$ a área mista. Então,

$$
A_{01}^{2} \geq A_{0} A_{1}
$$

sendo válida a igualdade se, e somente se, as curvas $c_{0}$ e $c_{1}$ são homotéticas, isto é, diferem apenas por uma dilatação ou translação.

Prova.

Sem perda de generalidade podemos supor que a origem esteja nos interiores das regiões limitadas pelas curvas $c_{0}$ e $c_{1}$ (conforme já dissemos, ela será chamada de centro destas curvas). Fixe um número $r$ tal que $r_{0} \leq r \leq R_{0}$. 


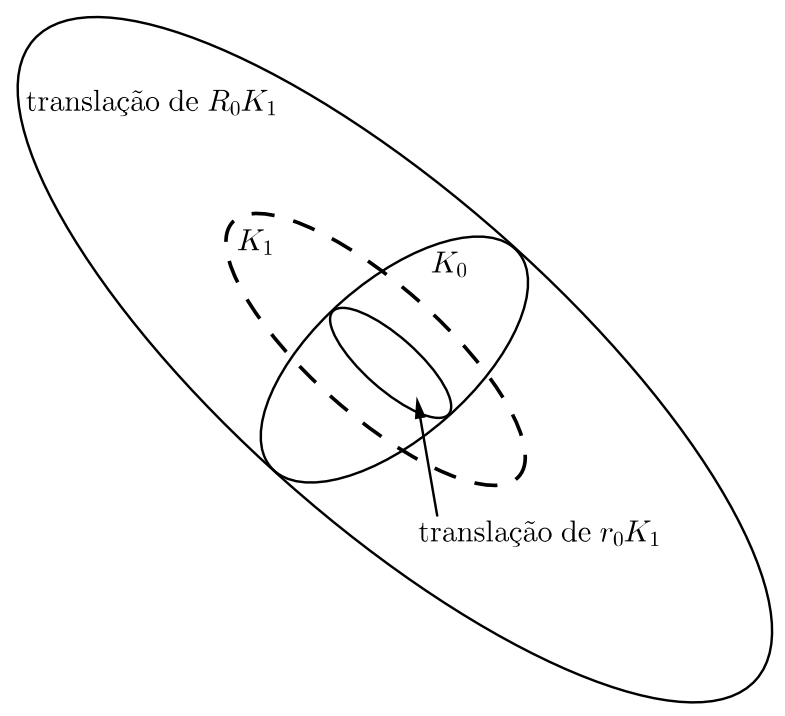

Figura 3.4: Translações de $r_{0} K_{1}$ e $R_{0} K_{1}$.

Dado um ponto $\alpha$ do plano, considere a translação $\alpha+r c_{1}$ de $r c_{1}$. Defina a função $N(\alpha)$ como o número de pontos de interseção entre as curvas $c_{0}$ e a translação $\alpha+r c_{1}$. Isto é,

$$
N(\alpha)=\#\left(\left(\alpha+r c_{1}\right) \cap c_{0}\right)
$$

Agora considere a seguinte expressão

$$
I=\iint_{\mathbb{R}^{2}} N(\alpha) d x d y
$$

Note que $N(\alpha)$ pode assumir o valor infinito quando a translação $\alpha+r c_{1}$ tangenciar (externamente ou internamente) $c_{0}$ conforme mostra a figura 3.5. Contudo, os conjuntos dos pontos $\alpha$ que tornam isto possível são também curvas, que possuem área zero, logo têm valor zero na integral 3-6.

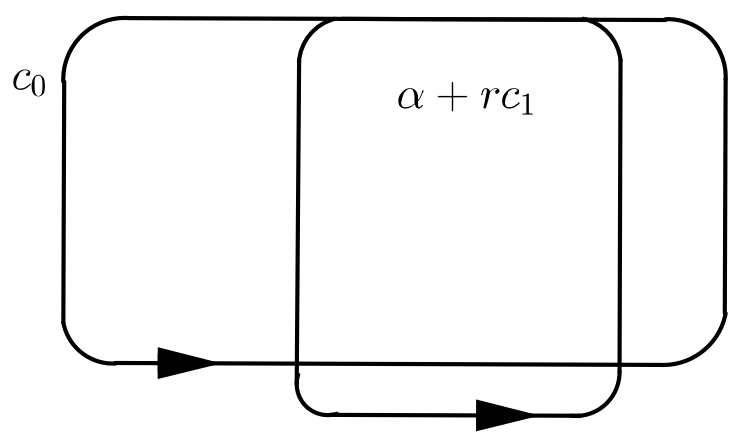

Figura 3.5: Duas curvas podem ter infinitos pontos de interseção. 
Por outro lado, o lugar geométrico dos pontos $\alpha$ tal que $\alpha+r c_{1}$ tangencia externamente $c_{0}$ é exatamente a curva $c_{2}$ do lema 3.2 se trocarmos a translação de $c_{1}$ por $\alpha+r c_{1}$, conforme mostra a figura 3.6. Logo, se $\alpha$ for um ponto exterior à região limitada por $c_{2}$, então $N(\alpha)=0$.

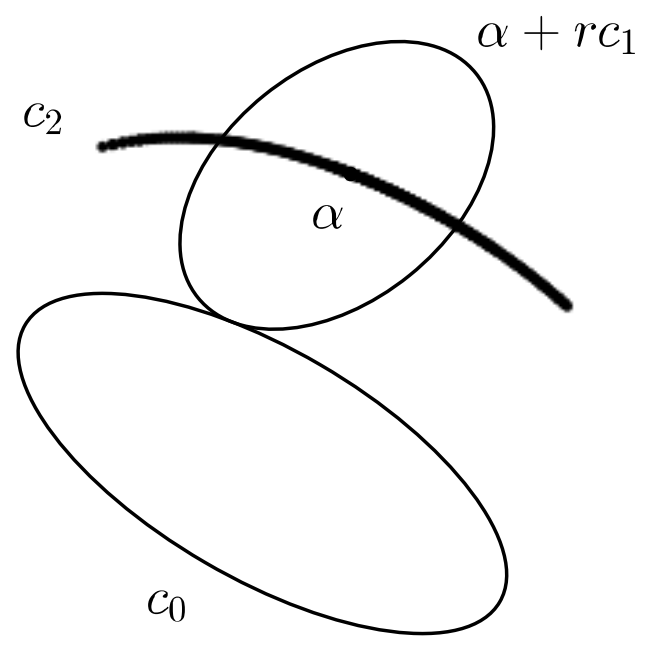

Figura 3.6: Translações de $r c_{1}$ que tangenciam externamente a curva $c_{0}$.

Além disso, nenhuma translação de $r K_{1}$ pode conter $K_{0}$ inteiramente (sem que suas fronteiras sejam tangentes), pois $r \leq R_{0}$. Analogamente nenhuma translação de $r K_{1}$ pode estar contida inteiramente em $K_{0}$ (sem que suas fronteiras sejam tangentes), pois $r_{0} \leq r$.

Pelo lema 3.2 aplicado a $c_{0}$ e $r c_{1}$, segue que:

$$
A_{2}=A_{0}+2 r A_{01}^{*}+r^{2} A_{1}
$$

Por todas estas razões, se $K_{2}$ é a região limitada por $c_{2}$, concluímos:

$$
I=\iint_{\mathbb{R}^{2}} N(\alpha) d x d y=\iint_{K_{2}} N(\alpha) d x d y \geq \iint_{K_{2}} 2 d x d y \geq 2 A_{2}
$$

Isto é,

$$
I \geq 2\left(A_{0}+2 r A_{01}^{*}+r^{2} A_{1}\right)
$$


A relação 3-7 nos fornece uma cota inferior de $I$. Agora vamos calcular o valor exato de $I$. Isto será feito em três etapas:

1. Vamos determinar o lugar geométrico dos centros de todas as translações de $r c_{1}$ que contêm um ponto $\alpha_{0}$ dado;

2. Vamos determinar a área do lugar geométrico dos centros de todas as translações de $r c_{1}$ que cruzam um segmento dado de comprimento $d s_{0}$;

3. Vamos inscrever um polígono na curva $c_{0}$ que aproxima $c_{0}$ e depois somar todas as áreas correspondentes a cada um dos lados (de tamanhos infinitesimais) do polígono.

Na primeira etapa, fixemos um ponto $\alpha_{0}$. Se transladarmos a curva $r c_{1}$ de maneira que um ponto $\alpha_{1}$ de $r c_{1}$ fique sobre o ponto $\alpha_{0}$, então a origem será transladada para o ponto $\alpha_{0}-\alpha_{1}$. Como esta ideia vale para qualquer ponto $\alpha_{1}(\theta) \in r c_{1}$, podemos dizer que o lugar geométrico das origens transladadas é a curva $\alpha_{0}-\alpha_{1}(\theta)=\alpha_{0}-r c_{1}$, que é a reflexão em torno da origem da curva $r c_{1}$ transladada para o ponto $\alpha_{0}$ conforme mostra a figura 3.7. Logo, com relação a esta reflexão e translação, $\alpha_{0}$ é o ponto correspondente à origem que chamaremos de centro desta nova curva.

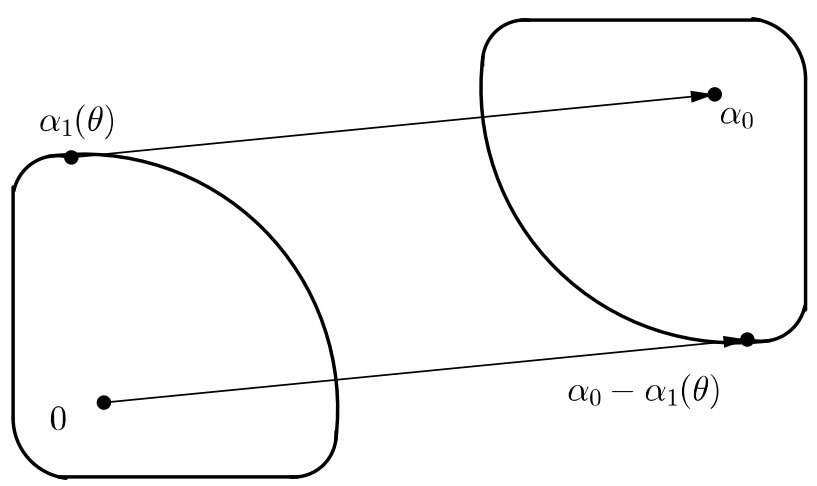

Figura 3.7: Lugar geométrico das origens transladadas de modo que $r c_{1}$ contenha o ponto $\alpha_{0}$.

Agora, na segunda etapa, vamos considerar um segmento de comprimento infinitesimal $d s_{0}$ aproximadamente contido na curva $c_{0}$. A priori queremos determinar todas as translações de $r c_{1}$ que intersectam este segmento. Por analogia ao que foi feito acima, basta considerarmos que cada ponto $\alpha$ do segmento é um ponto $\alpha_{0}$ e, portanto, o segmento será o lugar geométrico dos centros das curvas $\alpha-r c_{1}$. Assim, todas as curvas que representam os centros das translações de $r c_{1}$ que cruzam o segmento apenas uma vez se movem 
na direção do segmento. Portanto, o lugar geométrico de todos os centros destas curvas está compreendido em duas faixas hachuradas conforme mostra a figura 3.8. Pelo princípio de Cavalieri, cada uma destas faixas têm a mesma área de um retângulo de dimensões $d s_{0}$ e $r\left[p_{1}(\theta)+p_{1}(\theta+\pi)\right]$. Além disso, como na verdade o segmento não está necessariamente contido na curva, há um erro de ordem de grandeza menor em cada dimensão deste retângulo, que denotaremos por $O\left(d s_{0}\right)$. Logo, a contribuição de cada segmento à integral $I$ será:

$$
2 r\left[p_{1}(\theta)+p_{1}(\theta+\pi)\right] d s_{0}+O\left(d s_{0}\right)^{2}
$$

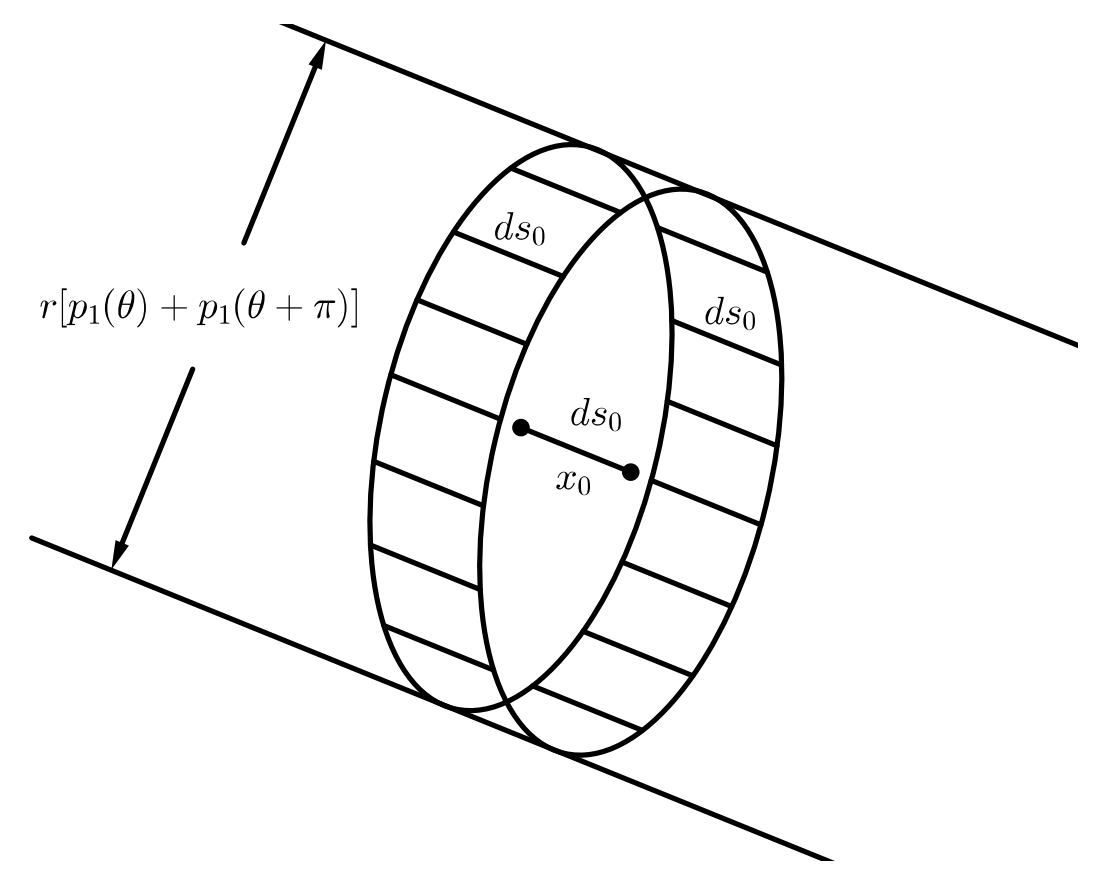

Figura 3.8: Os centros das translações de $r c_{1}$ pertencem às duas faixas hachuradas.

Finalmente na terceira etapa, somamos e passamos o limite para obtermos a fórmula:

$$
I=2 r \int_{0}^{2 \pi}\left[p_{1}(\theta)+p_{1}(\theta+\pi)\right] d s_{0}
$$

De acordo com o lema 3.2 e com a fórmula 3-3, temos:

$$
I=4 r\left(A_{01}+A_{01}^{*}\right)
$$

Por 3-9 e 3-7, temos $4 r\left(A_{01}+A_{01}^{*}\right) \geq 2\left(A_{0}+2 r A_{01}^{*}+r^{2} A_{1}\right)$ e concluímos que, para todo $r$ tal que $r_{0} \leq r \leq R_{0}$, temos:

$$
r^{2} A_{1}-2 r A_{01}+A_{0} \leq 0
$$


A existência de um número real $r$ que satisfaça a desigualdade acima significa que o polinômio quadrático $p(r)=A_{1} r^{2}-2 A_{01} r+A_{0}$ tem pelo menos um zero real. Isto quer dizer que o discriminante é positivo ou zero, donde $4 A_{01}^{2}-4 A_{0} A_{1} \geq 0 \Rightarrow A_{01}^{2} \geq A_{0} A_{1}$ e obtemos o resultado esperado.

Além disso, a desigualdade 3-10 é válida em particular para $r=r_{0}$ ou $r=R_{0}$. Se $A_{01}^{2}=A_{0} A_{1}$, então o discriminante é nulo. Assim, $r_{0}=R_{0}$ é zero duplo do polinômio e, portanto, $c_{0}$ e $c_{1}$ são homotéticas. Por outro lado, se $c_{0}$ e $c_{1}$ são homotéticas, então $A_{0}=r_{0}^{2} A_{1}$ e $A_{01}=\frac{1}{2} \int_{0}^{2 \pi}\left[p_{0} p_{1}-p_{0}^{\prime} p_{1}^{\prime}\right] d \theta=$ $\frac{r_{0}}{2} \int_{0}^{2 \pi}\left[p_{1}^{2}-p_{1}^{\prime 2}\right] d \theta=r_{0} A_{1}$. Logo, $A_{01}^{2}=r_{0}^{2} A_{1}^{2}=A_{0} A_{1}$. Desta forma, provamos que $A_{01}^{2}=A_{0} A_{1}$ se, e somente se, $c_{0}$ e $c_{1}$ são homotéticas.

Corolário 3.1.1 (Desigualdade isoperimétrica clássica) No plano euclideano, se $L_{0}$ é o comprimento de uma curva convexa fechada $c_{0}$, que limita uma região de área $A_{0}$. Então,

$$
\frac{1}{4} L_{0}^{2} \geq \pi A_{0}
$$

Prova. Basta considerar que $c_{1}$ seja o círculo unitário. Assim, na norma euclideana $A_{1}=\pi, p_{1}=1$ e, pela fórmula 3-3, temos $A_{01}=\frac{1}{2} \oint d s_{0}=\frac{1}{2} L_{0}$. Então, aplicamos o corolário 3.1 e segue o resultado.

O teorema 3.1 e o corolário 3.1.1 nos dizem que, no plano euclideano, dentre todas as curvas convexas fechadas de comprimento fixo $L_{0}$, a que delimita a maior área é a curva homotética ao círculo unitário, isto é, um círculo de raio $\frac{L_{0}}{2 \pi}$. De fato, se $A_{c}$ é a área do círculo cujo perímetro é $L_{0}$, então o raio deste círculo é $\frac{L_{0}}{2 \pi}$ e $A_{c}=\pi \cdot\left(\frac{L_{0}}{2 \pi}\right)^{2}=\frac{L_{0}^{2}}{4 \pi}$. Como $\frac{L_{0}^{2}}{4} \geq \pi A_{0}$, temos

$$
A_{c}=\frac{L_{0}^{2}}{4 \pi} \geq A_{0}
$$

Sendo que haverá igualdade se, e somente se, $c_{0}$ for homotética ao círculo unitário, isto é, quando $c_{0}$ for o círculo de raio $\frac{L_{0}}{2 \pi}$.

Veremos no próximo capítulo que, contrariando nossa intuição, se considerarmos o plano com uma norma não euclideana, a resposta para este problema não será mais necessariamente o círculo, mas uma curva conhecida como isoperimetrix. 


\section{4}

\section{O plano de Minkowski}

O objetivo principal deste capítulo é determinar, dentre todas as curvas convexas, fechadas e simples de perímetro fixo em um plano de Minkowski, qual é aquela que delimita a maior área. O caso do plano euclideano já foi resolvido no capítulo anterior. Para estendermos a solução deste problema, conhecido como problema isoperimétrico, para planos munidos com uma norma qualquer, teremos antes que estudar alguns conceitos fundamentais. Segundo Thompson [1], podemos definir:

Definição 4.1 Um espaço de Minkowski é um espaço vetorial normado de dimensão finita.

Neste trabalho iremos estudar especificamente espaços de Minkowski de dimensão 2, também chamados de planos de Minkowski, que serão denotados a partir de agora por $\mathcal{M}$. Quando quisermos considerar uma norma específica, ela será explicitada.

\section{1}

\section{Bolas no plano de Minkowski}

Nesta seção veremos que normas determinam bolas e vice-versa. Portanto, bolas são conjuntos fundamentais para definirmos a maneira como vamos medir distâncias no plano de Minkowski. As ideias a seguir são baseadas em Thompson [1].

Definição 4.2 Uma bola de centro em c e raio $r$ em $\mathcal{M}$ é o conjunto

$$
B_{r}(c)=\{x \in \mathcal{M}:\|x-c\| \leq r\}
$$

Denominamos de bola unitária em $\mathcal{M}$, que denotaremos simplesmente por $B$, a bola de centro na origem e raio 1 , isto é, o conjunto $B_{1}(0)$. A fronteira de $B_{r}(c)$ é também chamada de círculo em $\mathcal{M}$ e é definida por

$$
\partial B_{r}(c)=\{x \in \mathcal{M}:\|x-c\|=r\}
$$


O círculo unitário em $\mathcal{M}$ é o conjunto $\partial B_{1}(0)$, que denotaremos simplesmente por $\partial B$. Os exemplos a seguir nos mostram que a forma geométrica da bola depende da norma escolhida.

Exemplo 4.1 A figura 4.1 mostra os respectivos círculos unitários (de dentro para fora) referentes às normas $\ell_{p}$ para $p=1, \frac{3}{2}, 2,4,10$ e $\infty$.

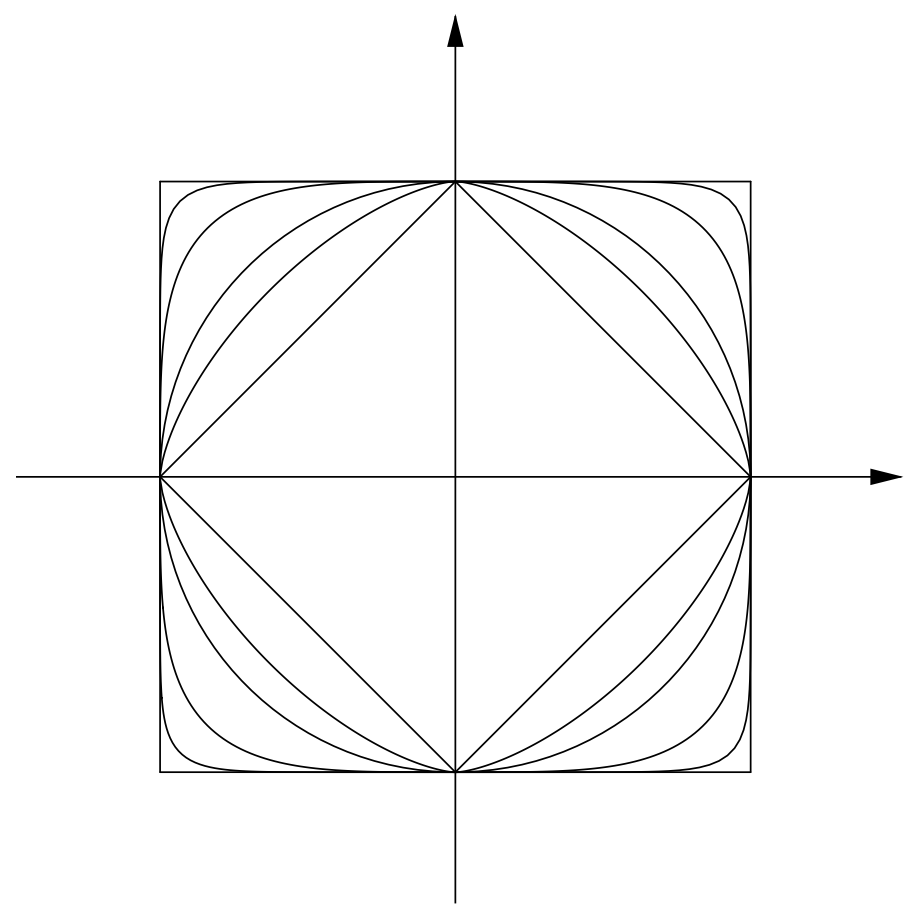

Figura 4.1: Círculos unitários para normas $\ell_{p}$.

Definição 4.3 Sejam $\mathcal{M}$ e $\mathcal{N}$ planos de Minkowski com normas $\|.\| \|_{\mathcal{M}}$ e $\|\cdot\|_{\mathcal{N}}$ respectivamente. Uma isometria de $\mathcal{M}$ em $\mathcal{N}$ é uma aplicação $T$ tal que para todo $x_{1}, x_{2} \in \mathcal{M}$ temos

$$
\left\|x_{1}-x_{2}\right\|_{\mathcal{M}}=\left\|T\left(x_{1}\right)-T\left(x_{2}\right)\right\|_{\mathcal{N}}
$$

Teorema 4.1 Sejam $\mathcal{M}$ e $\mathcal{N}$ os conjuntos definidos acima. Se uma transformação linear $T$ é uma isometria de $\mathcal{M}$ em $\mathcal{N}$, então

(i) $T\left(B_{\mathcal{M}}\right)=B_{\mathcal{N}}$

(ii) $T\left(\partial B_{\mathcal{M}}\right)=\partial B_{\mathcal{N}}$

(iii) Se F é um subconjunto extremo de $B_{\mathcal{M}}$, então $T(F)$ é um subconjunto extremo de $B_{\mathcal{N}}$

onde $B_{\mathcal{M}}$ e $B_{\mathcal{N}}$ denotam as respectivas bolas unitárias de $\mathcal{M}$ e $\mathcal{N}$. 
Prova.

(i) Como $T$ é linear e uma isometria, temos

$$
\|x-0\|_{\mathcal{M}}=\|T(x)-T(0)\|_{\mathcal{N}}=\|T(x)-0\|_{\mathcal{N}}
$$

Logo, $x \in B_{\mathcal{M}}$ se, e somente se, $T(x) \in B_{\mathcal{N}}$.

(ii) Analogamente pelas igualdades acima, $x \in \partial B_{\mathcal{M}}$ se, e somente se, $T(x) \in \partial B_{\mathcal{N}}$.

(iii) Suponhamos que $F$ seja um subconjunto extremo de $B_{\mathcal{M}}$ e que $y \in T(F)$ com $y=\alpha y_{1}+(1-\alpha) y_{2}$ para algum $y_{1}, y_{2} \in B_{\mathcal{N}}$. Então $y=T(x)$ para $x \in F$ e, pelo item (i), $y_{i}=T\left(x_{i}\right)$ para $x_{i} \in B_{\mathcal{M}}(\operatorname{com} i \in\{1,2\})$.

Afirmamos que $T$ possui uma inversa $T^{-1}$. De fato, toda isometria é injetiva pois se $T(a)=T(b) \Rightarrow\|a-b\|_{\mathcal{M}}=\|T(a)-T(b)\|_{\mathcal{N}}=0$, donde $a=b$. Pelo teorema do núcleo e da imagem, como $\mathcal{M}$ e $\mathcal{N}$ têm dimensão 2 , segue que $T$ também é sobrejetiva.

Como $T^{-1}$ é linear, temos

$$
\begin{gathered}
T^{-1}(y)=T^{-1}\left(\alpha y_{1}+(1-\alpha) y_{2}\right) \\
x=\alpha T^{-1}\left(y_{1}\right)+(1-\alpha) T^{-1}\left(y_{2}\right) \\
x=\alpha x_{1}+(1-\alpha) x_{2}
\end{gathered}
$$

Pela definição $2.3 x_{1}, x_{2} \in F$ e, portanto, $y_{1}, y_{2} \in T(F)$ como queríamos.

Teorema 4.2 A bola unitária $B$ em $\mathcal{M}$ é um conjunto simétrico e convexo. Além disso, com relação à topologia induzida pela norma, B é um conjunto fechado e a origem é um ponto interior.

Prova. De fato, pela definição de norma 2.13, temos que $\|-x\|=\|(-1) x\|=$ $|-1|\|x\|=\|x\|$ e segue a simetria de $B$. Dados $x, y \in B$ e $0 \leq \alpha \leq 1$, então

$$
\|\alpha x+(1-\alpha) y\| \leq \alpha\|x\|+(1-\alpha)\|y\| \leq \alpha+(1-\alpha)=1
$$

pois $\|x\| \leq 1,\|y\| \leq 1$ e pelos axiomas satisfeitos pela norma. Logo, $\alpha x+(1-\alpha) y \in B$ e $B$ é convexo. A desigualdade $|\|x\|-\|y\|| \leq\|x-y\|$ implica que a norma é uma função uniformemente contínua e, portanto, contínua. Como $B$ é a imagem inversa do conjunto [0,1], que é fechado, então $B$ também 
é fechado. Evidentemente 0 pertence ao aberto $\{x:\|x\|<1\} \subset B$ e assim vemos que 0 é um ponto interior de $B$.

Por outro lado, se $B$ é um conjunto simétrico e convexo de $\mathcal{M}$ tal que a interseção entre ele e cada reta que passa pela origem é um segmento limitado e fechado de comprimento positivo, então podemos definir um funcional $\|\cdot\|_{B}$ sobre $\mathcal{M}$ por

$$
\|x\|_{B}=\inf \left\{\xi \in \mathbb{R}^{+}: x \in \xi B\right\}
$$

Definição 4.4 O funcional \|\|$_{B}$ é chamado o funcional de Minkowski de $B$.

Exemplo 4.2 A figura 4.2 nos ajuda a entender a definição acima. Sejam dois vetores $x$ e $y$ do plano tais que $\|x\|_{2}=\|y\|_{2}$. Seja $B$ a bola unitária relativa à norma $\ell_{10}$. Pela definição, a figura nos mostra que $\|x\|_{B}=1,64$ e $\|y\|_{B}=2,06$. Também é possível verificar que $\|u\|_{B}=\|u\|_{10}$ para qualquer $u$.

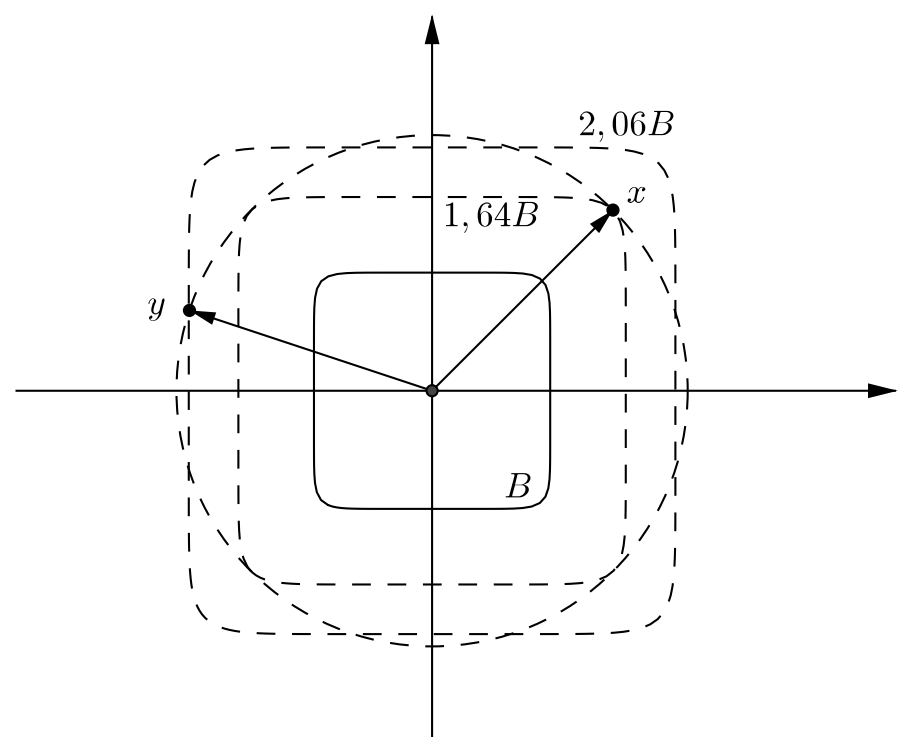

Figura 4.2: Dois vetores tais que $\|x\|_{2}=\|y\|_{2}$, mas $\|x\|_{B} \neq\|y\|_{B}$.

Geometricamente também podemos entender a definição acima da seguinte forma: trace a semi-reta a partir da origem 0 que passa pelo ponto $x$ de $\mathcal{M}$. Pela definição de $B$, sempre existe um ponto $u$ que é a interseção desta reta com $\partial B$. O segmento $0 u$ representa a unidade de medida para se medir a distância entre a origem e o ponto $x$ conforme mostra a figura 4.3. Isto é, para todo $x \in \mathcal{M}$, podemos escrever $x=t u$ para algum $t \geq 0$ e $u \in \partial B$, as$\operatorname{sim}\|x\|_{B}=t$. Note que, dependendo da escolha de $B$, teremos possivelmente diferentes unidades de medida de comprimento para diferentes direções. O teorema a seguir nos garante que realmente podemos medir comprimentos desta forma. 


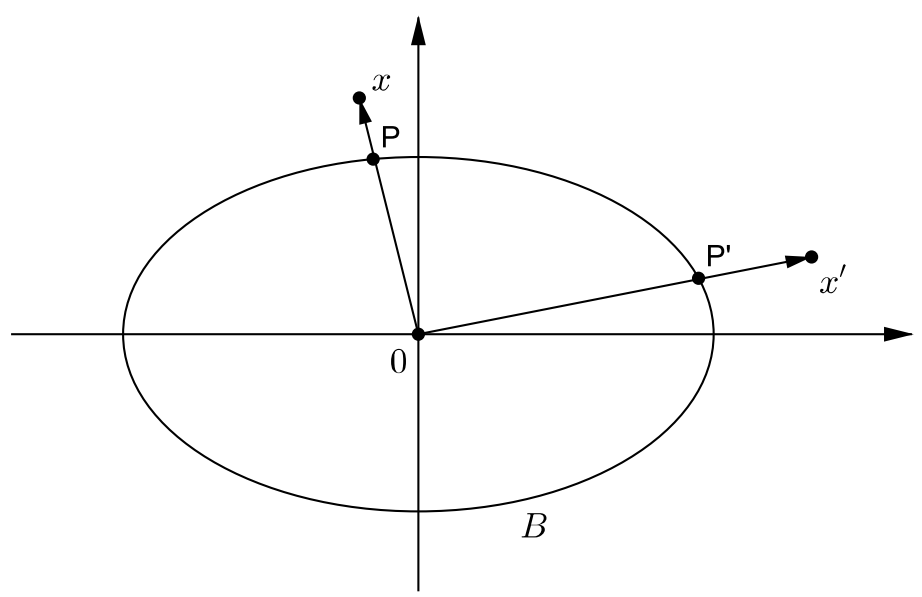

Figura 4.3: Diferentes unidades de comprimento para diferentes direções.

Teorema 4.3 Se B é um conjunto simétrico e convexo de $\mathcal{M}$ tal que a interseção entre ele e cada reta que passa pela origem é um segmento (nãotrivial) limitado e fechado, então $\|.\|_{B}$ é uma norma sobre $\mathcal{M}$.

Prova. Por definição, $\|x\|_{B} \geq 0$. Por hipótese, se $x \neq 0$ então existe uma semireta partindo da origem 0 que passa por $x$ e que cruza $\partial B$ em um ponto $\alpha x$ com $0<\alpha<\infty$. Assim, por definição, $\|x\|_{B}=\alpha^{-1}>0$ e segue que $\|x\|_{B}$ é um número estritamente positivo. Isto prova que $\|\cdot\|_{B}$ satisfaz o primeiro axioma de norma (ver definição 2.13).

Se $\beta \geq 0$, então $\|\beta x\|_{B}=\beta\|x\|_{B}=|\beta|\|x\|_{B}$ por definição. Se $\beta<0$, então $\|\beta x\|_{B}=\|-\beta(-x)\|_{B}=-\beta\|-x\|_{B}=-\beta\|x\|_{B}=|\beta|\|x\|_{B}$, pois $-\beta>0$ e $B$ é simétrico. Em qualquer caso, segue o segundo axioma de norma.

Agora vamos provar que $\|.\|_{B}$ satisfaz à desigualdade triangular (terceiro axioma de norma). Suponhamos $x$ e $y$ elementos distintos da origem de $\mathcal{M}$. Então, tomemos os vetores $\hat{x}=\frac{x}{\|x\|_{B}}$ e $\hat{y}=\frac{y}{\|y\|_{B}}$. Evidentemente por 4-1, $\hat{x} \mathrm{e}$ $\hat{y}$ pertencem à $B$, pois $\|\hat{x}\|_{B}=\|\hat{y}\|_{B}=1$ (pelo segundo axioma já provado) e, por hipótese, a interseção entre a reta suporte de $\overline{0 x}$ (por exemplo) e $B$ é um segmento de reta fechado com extremos $-\hat{x}$ e $\hat{x}$. Existe um vetor $z$ que pode ser escrito da seguinte forma:

$$
z=\frac{\|x\|_{B}}{\|x\|_{B}+\|y\|_{B}} \hat{x}+\frac{\|y\|_{B}}{\|x\|_{B}+\|y\|_{B}} \hat{y}=\left(\|x\|_{B}+\|y\|_{B}\right)^{-1}(x+y)
$$

Logo, pela convexidade de $B, z \in B$, pois $\frac{\|x\|_{B}}{\|x\|_{B}+\|y\|_{B}}+\frac{\|y\|_{B}}{\|x\|_{B}+\|y\|_{B}}=1$. Isto é, por $4-1,\|z\|_{B} \leq 1$. Como $\|z\|_{B}=\left(\|x\|_{B}+\|y\|_{B}\right)^{-1}\|x+y\|_{B}$, temos:

$$
\left(\|x\|_{B}+\|y\|_{B}\right)^{-1}\|x+y\|_{B} \leq 1 \Rightarrow\|x+y\|_{B} \leq\|x\|_{B}+\|y\|_{B}
$$


Esta desigualdade é trivial no caso em que $x=0$ ou $y=0$.

Os teoremas 4.2 e 4.3 mostram que há uma correspondência biunívoca entre normas sobre $\mathcal{M}$ e os conjuntos convexos, simétricos e fechados com interior não-vazio em $\mathcal{M}$. Esta correspondência faz da convexidade uma propriedade essencial no estudo dos planos de Minkowski. Assim, qualquer um daqueles conjuntos pode ser escolhido para ser a bola unitária destes planos se tomarmos o funcional de Minkowski como norma.

A partir de agora a métrica escolhida será a métrica usual vista na seção 2.2, isto é, dados quaisquer dois pontos $x, y \in \mathcal{M}$, a distância entre $x$ e $y$ será dada por $d(x, y)=\|x-y\|_{B}$.

Exemplo 4.3 Seja $B$ a região fechada de $\mathcal{M}$ cuja fronteira é uma elipse conforme mostra a figura 4.4. Evidentemente $B$ é convexa e simétrica com interior não-vazio. Na figura vemos um exemplo de dois pontos $x$ e y cuja distância é 3 na norma $\|.\|_{B}$. A determinação do valor de $\|x-y\|_{B}$ é feita conforme descrito anteriormente.

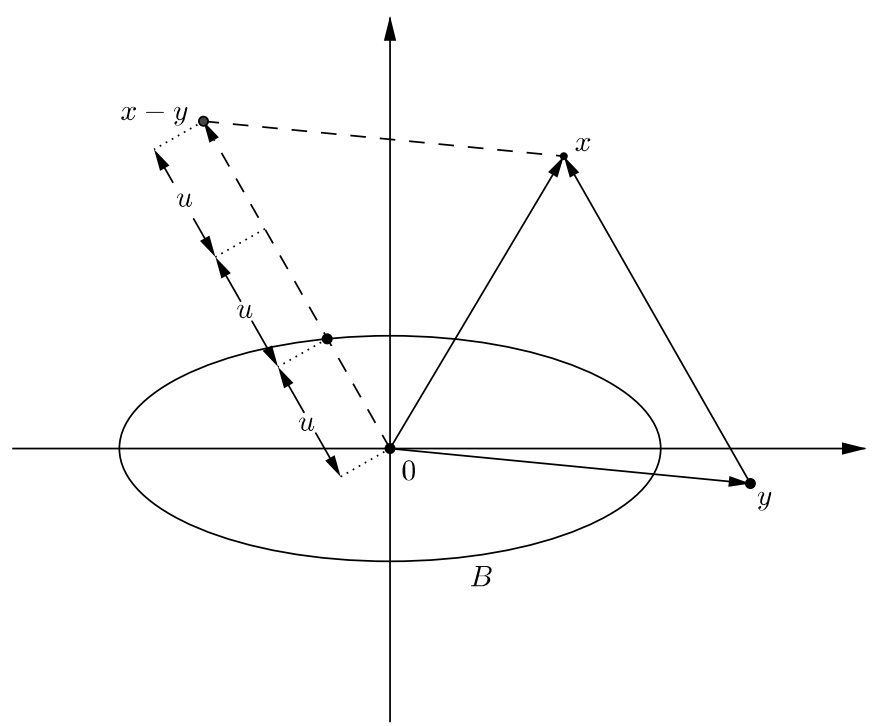

Figura 4.4: A distância entre dois pontos.

Exemplo $4.4 \mathrm{Na}$ figura 4.5 podemos ver mais alguns exemplos de bolas unitárias em planos de Minkowski. Todos os conjuntos são convexos, simétricos e com interiores não-vazios, contudo suas fronteiras não são curvas necessariamente suaves. Cada bola vai determinar naturalmente uma norma distinta a partir do funcional de Minkowski. 


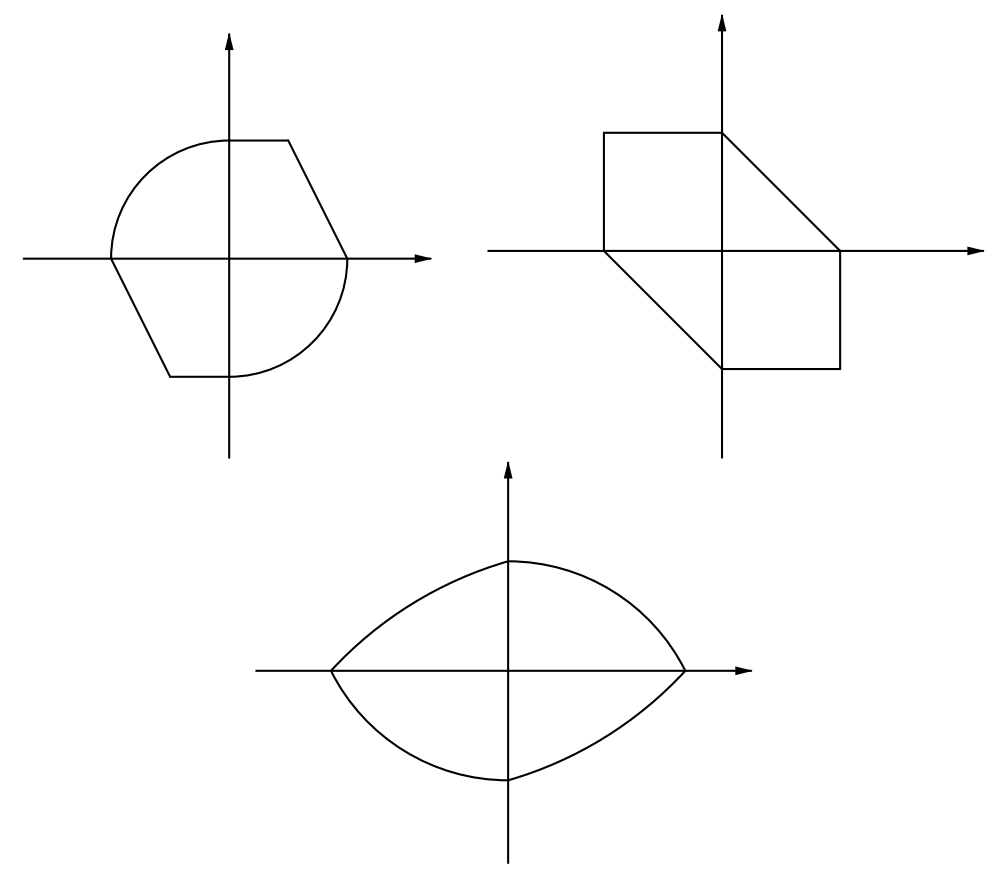

Figura 4.5: Exemplos de bolas.

\section{2}

O dual

Agora vamos considerar a norma sobre o espaço dos funcionais lineares limitados sobre $\mathcal{M}$ do exemplo 2.14 da seção 2.2

$$
\|f\|=\sup \{|f(x)| ; x \in B\}
$$

Conforme vimos na seção 2.3, o teorema 2.3 nos diz que transformações lineares definidas sobre espaços de Minkowski são contínuas e, portanto, quando restritas à conjuntos compactos como a bola unitária são limitadas. Em particular, funcionais lineares restritos à bola unitária de um plano de Minkowski são limitados. Logo, (4-2) define uma norma sobre o plano dual $\mathcal{M}^{*}$ de todos os funcionais lineares sobre $\mathcal{M}$. Podemos, então, definir a bola unitária de $\mathcal{M}^{*}$ por

Definição 4.5 A bola unitária de $\mathcal{M}^{*}$ é o conjunto

$$
B^{\circ}=\left\{f \in \mathcal{M}^{*}:\|f\| \leq 1\right\}
$$

Este conjunto é também chamado de bola dual. A norma associada à bola dual é chamada norma dual.

Agora vamos considerar que o plano $\mathcal{M}$ esteja na base canônica $\left\{e_{1}, e_{2}\right\}$ e o plano dual $\mathcal{M}^{*}$ esteja na base dual (usual) $\left\{e_{1}^{*}, e_{2}^{*}\right\}$. Um funcional linear 
$f$ será escrito em coordenadas $\left(\phi_{1}, \phi_{2}\right)$ e vetores $x \in \mathcal{M}$ serão escritos como $\left(\alpha_{1}, \alpha_{2}\right)$ (ambos em relação às bases acima mencionadas).

Teorema 4.4 A norma dual da norma $\ell_{p}$ é a norma $\ell_{q}$, onde $\frac{1}{p}+\frac{1}{q}=1$.

Prova. Primeiro vamos provar o caso em que $1<p \neq \infty$.

Se $x \in \mathcal{M}$ e $f \in \mathcal{M}^{*}$, então pela desigualdade de Hölder temos

$$
|f(x)|=\left|\phi_{1} \alpha_{1}+\phi_{2} \alpha_{2}\right| \leq\left(\left|\alpha_{1}\right|^{p}+\left|\alpha_{2}\right|^{p}\right)^{1 / p} \cdot\left(\left|\phi_{1}\right|^{q}+\left|\phi_{2}\right|^{q}\right)^{1 / q}
$$

onde $q$ é tal que $\frac{1}{p}+\frac{1}{q}=1$.

Suponhamos $\|f\|_{q} \leq 1$. Então, a desigualdade acima fica

$$
|f(x)|=\left|\phi_{1} \alpha_{1}+\phi_{2} \alpha_{2}\right| \leq\left(\left|\alpha_{1}\right|^{p}+\left|\alpha_{2}\right|^{p}\right)^{1 / p}=\|x\|_{p}
$$

Desta forma, sempre que $\|x\|_{p} \leq 1$, teremos $|f(x)| \leq 1$. Então, considerando a norma (4-2), isto significa que $\|f\| \leq 1$. Isto é, provamos que $\|f\|_{q} \leq 1$ implica $\|f\| \leq 1$.

Por outro lado, vamos supor $\|f\| \leq 1$. Para $i \in\{1,2\}$, considere a função

$$
\operatorname{sgn} \phi_{i}= \begin{cases}1 & \text { se } \phi_{i}>0 \\ 0 & \text { se } \phi_{i}=0 \\ -1 & \text { se } \phi_{i}<0\end{cases}
$$

Podemos definir as coordenadas de $x$ como $\left(\alpha_{1}, \alpha_{2}\right)=\left(\operatorname{sgn} \phi_{1}\left|\phi_{1}\right|^{q-1}, \operatorname{sgn} \phi_{2}\left|\phi_{2}\right|^{q-1}\right)$ e também $\tilde{x}=\|x\|_{p}^{-1} x$. Assim,

$$
\begin{aligned}
\|x\|_{p} & =\left(\left|\alpha_{1}\right|^{p}+\left|\alpha_{2}\right|^{p}\right)^{1 / p} \\
& =\left(\left|\phi_{1}\right|^{p(q-1)}+\left|\phi_{2}\right|^{p(q-1)}\right)^{1 / p} \\
& =\left(\left|\phi_{1}\right|^{q}+\left|\phi_{2}\right|^{q}\right)^{1 / p} \\
& =\|f\|_{q}^{q / p}
\end{aligned}
$$

Pois $\frac{1}{p}+\frac{1}{q}=1 \Rightarrow p(q-1)=q$. Então, $\tilde{x}=\left(\tilde{\alpha_{1}}, \tilde{\alpha_{2}}\right)=\|f\|_{q}^{-q / p} x=$ $\|f\|_{q}^{-q / p}\left(\alpha_{1}, \alpha_{2}\right)$. É claro que $\|\tilde{x}\|_{p}=1$. Isso implica $\tilde{x} \in B$ e, como $\|f\| \leq 1$, temos

$$
\begin{aligned}
1 \geq f(\tilde{x}) & =\phi_{1} \tilde{\alpha}_{1}+\phi_{2} \tilde{\alpha_{2}}=\|f\|_{q}^{-q / p}\left[\phi_{1} \alpha_{1}+\phi_{2} \alpha_{2}\right] \\
& =\|f\|_{q}^{-q / p}\left[\phi_{1}\left(\operatorname{sgn} \phi_{1}\left|\phi_{1}\right|^{q-1}\right)+\phi_{2}\left(\operatorname{sgn} \phi_{2}\left|\phi_{2}\right|^{q-1}\right)\right] \\
& =\|f\|_{q}^{-q / p}\left[\left|\phi_{1}\right|^{q}+\left|\phi_{2}\right|^{q}\right]=\|f\|_{q}^{-q / p}\|f\|_{q}^{q} \\
& =\|f\|_{q}
\end{aligned}
$$


Na segunda linha, observamos que $\phi_{i} \operatorname{sgn} \phi_{i}=\left|\phi_{i}\right|($ para $i=1,2)$ e, na terceira linha, evidentemente $-\frac{q}{p}+q=1$.

Em suma, até aqui provamos que $\|f\| \leq 1$ se, e somente se, $\|f\|_{q} \leq 1$, isto é, $\|f\|=\|f\|_{q}$ para $1<p \neq \infty$.

Falta provar os casos $p=1$ e $p=\infty$. Seja $\mathcal{M}$ munido com a norma $\ell_{\infty}$ (a norma do máximo). Então, se $x \in \mathcal{M}$, temos $\|x\|_{\infty}=\max \left\{\left|\alpha_{1}\right|,\left|\alpha_{2}\right|\right\}$. Seja $f \in \mathcal{M}^{*}$, se $x \in B \Rightarrow\|x\|_{\infty} \leq 1$, logo

$$
\begin{aligned}
|f(x)|=\left|\phi_{1} \alpha_{1}+\phi_{2} \alpha_{2}\right| & \leq\left|\phi_{1} \alpha_{1}\right|+\left|\phi_{2} \alpha_{2}\right| \\
& \leq\|x\|_{\infty}\left(\left|\phi_{1}\right|+\left|\phi_{2}\right|\right) \\
& \leq\left|\phi_{1}\right|+\left|\phi_{2}\right|=\|f\|_{1}
\end{aligned}
$$

Isto é, se $\|f\|_{1} \leq 1$, então $|f(x)| \leq 1$ para todo $x \in B$ e, portanto, $\|f\| \leq 1$.

Por outro lado, se $f$ é tal que $\|f\| \leq 1$, vamos escolher $x_{f}=$ $\left(\operatorname{sgn} \phi_{1}, \operatorname{sgn} \phi_{2}\right)$. Evidentemente, $\left\|x_{f}\right\|_{\infty} \leq 1 \Rightarrow x_{f} \in B$ e $f\left(x_{f}\right) \leq 1$ por hipótese. Como $f\left(x_{f}\right)=\phi_{1} \operatorname{sgn} \phi_{1}+\phi_{2} \operatorname{sgn} \phi_{2}=\left|\phi_{1}\right|+\left|\phi_{2}\right|=\|f\|_{1}$, segue que $\|f\|_{1} \leq 1$. Enfim, provamos que $\|f\| \leq 1$ se, e somente se, $\|f\|_{1} \leq 1$, ou seja, $\|f\|=\|f\|_{1}$ e concluímos que a norma dual de $\ell_{\infty}$ é a norma $\ell_{1}$. Para terminarmos esta demonstração, basta provar a recíproca desta afirmação, mas não vamos provar isto agora porque o teorema 4.6 que veremos mais adiante vai nos garantir isto.

Para chegarmos ao teorema 4.6, antes precisamos da definição abaixo e da versão para espaços vetoriais de dimensão finita do teorema de Hahn-Banach.

Definição 4.6 Um funcional $h$ definido sobre um espaço vetorial $X$ é chamado sublinear quando

(i) $h\left(x_{1}+x_{2}\right) \leq h\left(x_{1}\right)+h\left(x_{2}\right), \forall x_{1}, x_{2} \in X$;

(ii) $h(\alpha x)=\alpha h(x), \forall x \in X, \alpha \geq 0$.

Exemplo 4.5 Uma norma é um funcional sublinear.

Teorema 4.5 (Hahn-Banach) Suponha que Y seja um subespaço (próprio) de um espaço vetorial $X$ de dimensão finita e que h seja um funcional sublinear definido sobre $X$. Se $f$ é um funcional linear definido sobre $Y$ tal que

$$
f(y) \leq h(y), \quad \forall y \in Y
$$

então existe um funcional linear $\tilde{f}$ definido sobre $X$ tal que

(i) $\tilde{f}(y)=f(y), \forall y \in Y$; 
(ii) $\tilde{f}(x) \leq h(x), \forall x \in X$.

Prova. Suponhamos $x_{0} \in X \backslash Y$ e seja

$$
Y_{0}=\operatorname{span}\left\{Y, x_{0}\right\}=\left\{y+\alpha x_{0}: y \in Y, \alpha \in \mathbb{R}\right\}
$$

Se $y_{1}$ e $y_{2}$ são pontos quaisquer de $Y$, temos

$$
\begin{aligned}
f\left(y_{1}\right)-f\left(y_{2}\right)=f\left(y_{1}-y_{2}\right) & \leq h\left(y_{1}-y_{2}\right)=h\left(\left(y_{1}+x_{0}\right)+\left(-x_{0}-y_{2}\right)\right) \\
& \leq h\left(y_{1}+x_{0}\right)+h\left(-x_{0}-y_{2}\right)
\end{aligned}
$$

Logo, $f\left(y_{1}\right)-h\left(y_{1}+x_{0}\right) \leq f\left(y_{2}\right)+h\left(-x_{0}-y_{2}\right), \forall y_{1}, y_{2} \in Y$.

A inequação acima implica que

$\sup \left\{f\left(y_{1}\right)-h\left(y_{1}+x_{0}\right): y_{1} \in Y\right\} \leq \inf \left\{f\left(y_{2}\right)+h\left(-x_{0}-y_{2}\right): y_{2} \in Y\right\}$

E podemos dizer que existe um número real $\beta_{0}$ tal que

$$
f\left(y_{1}\right)-h\left(y_{1}+x_{0}\right) \leq \beta_{0}, \quad \forall y_{1} \in Y
$$

e

$$
\beta_{0} \leq f\left(y_{2}\right)+h\left(-x_{0}-y_{2}\right), \quad \forall y_{2} \in Y
$$

Vamos definir $\tilde{f}_{0}$ sobre $Y_{0}$ por $\tilde{f}_{0}\left(y+\alpha x_{0}\right)=f(y)-\alpha \beta_{0}$. Tomando $y_{1}=\alpha^{-1} y$ na desigualdade $4-4$, temos

$$
f\left(\alpha^{-1} y\right)-\beta_{0} \leq h\left(\alpha^{-1} y+x_{0}\right)
$$

Se $\alpha>0$, então $f(y)-\alpha \beta_{0} \leq h\left(y+\alpha x_{0}\right)$. Analogamente na inequação 4-5 temos

$$
f\left(\alpha^{-1} y\right)-\beta_{0} \geq-h\left(-\alpha^{-1} y-x_{0}\right)
$$

Se $\alpha<0$, então

$$
f(y)-\alpha \beta_{0} \leq-\alpha h\left((-\alpha)^{-1} y+(-\alpha)^{-1}\left(\alpha x_{0}\right)\right)=h\left(y+\alpha x_{0}\right)
$$

Em qualquer caso, obtemos $\tilde{f}_{0}\left(y+\alpha x_{0}\right) \leq h\left(y+\alpha x_{0}\right)$ para todo $y \in Y$. Portanto, $\tilde{f}_{0}(x) \leq h(x)$ para todo $x \in Y_{0}$. Vemos facilmente que $\tilde{f}_{0}$ é um funcional linear, pois se $a, b \in Y_{0}$ então $a=y_{a}+\alpha_{a} x_{0}$ e $b=y_{b}+\alpha_{b} x_{0}$ para $y_{a}$, 
$y_{b} \in Y$ e $\alpha_{a}, \alpha_{b} \in \mathbb{R}$. Portanto, como $f$ é um funcional linear, temos

$$
\begin{aligned}
\tilde{f}_{0}(a+b) & =\tilde{f}_{0}\left(\left(y_{a}+\alpha_{a} x_{0}\right)+\left(y_{b}+\alpha_{b} x_{0}\right)\right)=\tilde{f}_{0}\left(\left(y_{a}+y_{b}\right)+\left(\alpha_{a}+\alpha_{b}\right) x_{0}\right) \\
& =f\left(y_{a}+y_{b}\right)+\left(\alpha_{a}+\alpha_{b}\right) \beta_{0}=f\left(y_{a}\right)+f\left(y_{b}\right)+\alpha_{a} \beta_{0}+\alpha_{b} \beta_{0} \\
& =\tilde{f}_{0}\left(y_{a}+\alpha_{a} x_{0}\right)+\tilde{f}_{0}\left(y_{b}+\alpha_{b} x_{0}\right) \\
& =\tilde{f}_{0}(a)+\tilde{f}_{0}(b)
\end{aligned}
$$

Seja $\lambda \in \mathbb{R}$, então

$$
\begin{aligned}
\tilde{f}_{0}(\lambda a) & =\tilde{f}_{0}\left(\lambda\left(y_{a}+\alpha_{a} x_{0}\right)\right)=\tilde{f}_{0}\left(\lambda y_{a}+\left(\lambda \alpha_{a} x_{0}\right)\right) \\
& =f\left(\lambda y_{a}\right)+\lambda \alpha_{a} \beta_{0}=\lambda\left[f\left(y_{a}\right)+\alpha_{a} \beta_{0}\right] \\
& =\lambda \tilde{f}_{0}(a)
\end{aligned}
$$

Além disso, para todo $y \in Y, \tilde{f}_{0}(y)=\tilde{f}_{0}\left(y+0 x_{0}\right)=f(y)+0 \beta_{0}=f(y)$ e assim verificamos que $\tilde{f}_{0}$ é a função que procurávamos definida sobre $Y_{0}$. Para verificar que podemos construir uma função com as propriedades acima definida sobre todo $X$, basta usarmos indução sobre a dimensão de $Y$. Sejam $m$ e $n$ respectivamente as dimensões de $Y$ e $X$. Se $n=m+1$, não há mais nada a fazer. Se $n>m+1$, já provamos que a função que buscamos existe definida sobre $Y_{0}$. Para $i \in\{1, \ldots, n-m-1\}$, suponhamos $x_{i} \in X \backslash Y_{i-1}$ e seja

$$
Y_{i}=\operatorname{span}\left\{Y_{i-1}, x_{i}\right\}=\left\{y+\alpha x_{i}: y \in Y_{i-1}, \alpha \in \mathbb{R}\right\}
$$

Suponha então que exista o funcional linear $\tilde{f}_{n-m-2}$ com as propriedades desejadas definida sobre $Y_{n-m-2}$. Por argumentos análogos, podemos verificar a existência de um número real $\beta_{n-m-1}$ e definir $\tilde{f}_{n-m-1}$ sobre $Y_{n-m-1}$ por

$$
\tilde{f}_{n-m-1}\left(y+\alpha x_{n-m-1}\right)=\tilde{f}_{n-m-2}(y)-\alpha \beta_{n-m-1}
$$

sendo $y \in Y_{n-m-2}$ e $x_{n-m-1} \in X \backslash Y_{n-m-2}$

Note que $Y_{n-m-1}=X$ e por razões análogas àquelas apresentadas acima $\tilde{f}_{n-m-1}$ é a função requerida e isto finaliza nossa demonstração.

Corolário 4.5.1 Se Y é um subespaço próprio de um espaço de Minkowski X e se $f$ é um funcional linear sobre $Y$, então existe um funcional linear $\tilde{f}$ sobre $X$ tal que

(i) $\tilde{f}(y)=f(y), \forall y \in Y$

(ii) $\|\tilde{f}\|_{X^{*}}=\|f\|_{Y^{*}}$ 
Prova. Seja $h(x)=\|f\|_{Y^{*}}\|x\|$. Como a norma é um funcional sublinear, a função $h$ assim definida sobre $X$ também o é. Sabemos que $|f(y)| \leq\|f\|_{Y^{*}}$ para todo $y \in B_{Y}$. Como $\frac{y}{\|y\|} \in B_{Y}$, então $f\left(\frac{y}{\|y\|}\right) \leq\left|f\left(\frac{y}{\|y\|}\right)\right| \leq\|f\|_{Y^{*}} \Rightarrow$ $\frac{f(y)}{\|y\|} \leq\|f\|_{Y^{*}} \Rightarrow f(y) \leq h(y)$. Pelo teorema 4.5, existe um funcional linear $\tilde{f}$ tal que $\tilde{f}(y)=f(y), \forall y \in Y$ e $\tilde{f}(x) \leq h(x)$ para todo $x \in X$. E podemos concluir que $\|\tilde{f}\|_{X^{*}}=\|f\|_{Y^{*}}$.

Corolário 4.5.2 (Hahn) Se $x_{0} \neq 0$ é um ponto pertencente a um espaço de Minkowski $X$, então existe um funcional linear $f_{0}$ sobre $X$ tal que $f_{0}\left(x_{0}\right)=$ $\left\|x_{0}\right\|$ e $f_{0}(x) \leq\|x\|$ para todo $x \in X$.

Prova. Seja $Y$ um subespaço vetorial unidimensional gerado por $x_{0}$ e defina o funcional linear $f$ sobre $Y$ por $f\left(\alpha x_{0}\right)=\alpha\left\|x_{0}\right\|$. Então, como todo elemento de $y \in Y$ pode ser escrito como $y=\alpha x_{0}$ para algum $\alpha \in \mathbb{R}$, se $\|y\|=\left\|\alpha x_{0}\right\| \leq 1$ então $|f(y)|=\left|f\left(\alpha x_{0}\right)\right|=|\alpha| \cdot\left\|x_{0}\right\|=\left\|\alpha x_{0}\right\| \leq 1$. Mas $\frac{x_{0}}{\left\|x_{0}\right\|} \in B_{Y}$ e $f\left(\frac{x_{0}}{\left\|x_{0}\right\|}\right)=1, \operatorname{logo}\|f\|_{Y^{*}}=1$. Seja $f_{0}$ o funcional cuja existência é garantida pelo corolário 4.5.1. Então $f_{0}\left(x_{0}\right)=f\left(x_{0}\right)=\left\|x_{0}\right\|$ e, tomando a função $h$ definida na prova daquele corolário por $h(x)=\|f\|_{Y^{*}}\|x\|$, como $\left\|f_{0}\right\|_{X^{*}}=\|f\|_{Y^{*}}=1$ temos $f_{0}(x) \leq\left\|f_{0}\right\|_{X^{*}}\|x\|=\|x\|$ para todo $x \in X$.

Corolário 4.5.3 Se x é um ponto de um espaço de Minkowski X, então

$$
\|x\|=\sup \left\{|f(x)|: f \in B^{\circ}\right\}
$$

Prova. Fixe um ponto $x \in X$. Se $x=0$, a igualdade acima é óbvia.

Vamos supor $x \neq 0$. Se $\|f\| \leq 1$ então $|f(x)| \leq\|f\| \cdot\|x\| \leq\|x\|$. Pelo corolário 4.5.2, existe um funcional linear $f_{0} \in B^{\circ}$ tal que $f_{0}(x)=\|x\|$. Isto é, $\sup \left\{|f(x)|: f \in B^{\circ}\right\}=\|x\|$.

Ao definirmos o plano dual $\mathcal{M}^{*}$, analogamente podemos considerar também $\mathcal{M}^{* *}=\left(\mathcal{M}^{*}\right)^{*}$. Cada vetor $x \in \mathcal{M}$ define um elemento $J x \in \mathcal{M}^{* *}$ tal que

$$
(J x)(f)=f(x)
$$

Se $J x=0$ então $f(x)=0$ para todo funcional linear $f \in \mathcal{M}^{*}$ e, portanto, $x=0$. Assim, a aplicação linear $J: \mathcal{M} \rightarrow \mathcal{M}^{* *}$ é injetiva. Como ambos $\mathcal{M}$ e $\mathcal{M}^{*}$ têm dimensão 2 , o mesmo ocorre com $\mathcal{M}$ e $\mathcal{M}^{* *}$ e, pelo teorema do núcleo e da imagem, a dimensão do conjunto $J(\mathcal{M}) \subset \mathcal{M}^{* *}$ também é 2 e segue que $J$ é sobrejetiva. Portanto, $\mathcal{M}$ e $\mathcal{M}^{* *}$ são isomorfos. 
Assim como (4-2) define uma norma sobre $\mathcal{M}^{*}$, analogamente também podemos definir uma norma sobre $\mathcal{M}^{* *}$

$$
\|\Phi\|=\sup \left\{|\Phi(f)|: f \in B^{\circ}\right\}, \quad \forall \Phi \in \mathcal{M}^{* *}
$$

Vamos mostrar agora que, além de existir um isomorfismo entre $\mathcal{M}$ e $\mathcal{M}^{* *}$, também existe uma isometria entre estes conjuntos.

Teorema 4.6 Se $\mathcal{M}^{* *}$ é munido da norma definida em 4-7, então o isomorfismo $J$ de $\mathcal{M}$ sobre $\mathcal{M}^{* *}$ é uma isometria.

Prova. Pela norma definida em 4-7 e pelo corolário 4.5.3, como $(J x)(f)=f(x)$, segue

$$
\|J x\|=\sup \left\{|J x(f)|: f \in B^{\circ}\right\}=\sup \left\{|f(x)|: f \in B^{\circ}\right\}=\|x\| .
$$

Segundo Thompson [1], este teorema pode ser chamado de teorema fundamental da geometria de Minkowski. Ele diz que os conjuntos $X$ e $X^{* *}$ são idênticos como espaços de Minkowski, isto é, além deles serem isomorfos, a distância entre dois pontos de um espaço é igual à distância entre o par de pontos correspondente no outro espaço e não há problema em ignorarmos o funcional $J$. Então, quando provamos no teorema 4.4 que a norma dual da norma $\ell_{\infty}$ é a norma $\ell_{1}$, o teorema 4.6 garante que reciprocamente a norma dual da norma $\ell_{1}$ é a norma $\ell_{\infty}$. E mais geralmente, podemos concluir que $\left(B^{\circ}\right)^{\circ}=B$.

\section{3}

\section{Normalidade no plano de Minkowski}

Nesta seção estudaremos a definição de normalidade, que será útil na seção 4.5 para a construção das curvas de Radon. O conceito de normalidade também será importante para estimarmos o comprimento da bola unitária no plano $\mathcal{M}$, o que será abordado no capítulo 5 . As ideias a seguir são baseadas em Thompson [1].

Teorema 4.7 Seja $\mathcal{A}$ um conjunto limitado e fechado de $\mathcal{M}$ que gera $\mathcal{M}$. Então existem pontos $x_{1}$ e $x_{2}$ em $\mathcal{A}$ e retas $r_{1}$ e $r_{2}$ tais que

(i) $x_{1} \in r_{1}$ e $x_{2} \in r_{2}$;

(ii) A reta $r_{1}$ é paralela ao $\operatorname{span}\left\{x_{2}\right\}$ e a reta $r_{2}$ é paralela ao $\operatorname{span}\left\{x_{1}\right\}$;

(iii) $A$ reta $r_{i}$ tangencia $\mathcal{A}$ no ponto $x_{i}$ para $i=1,2$. 


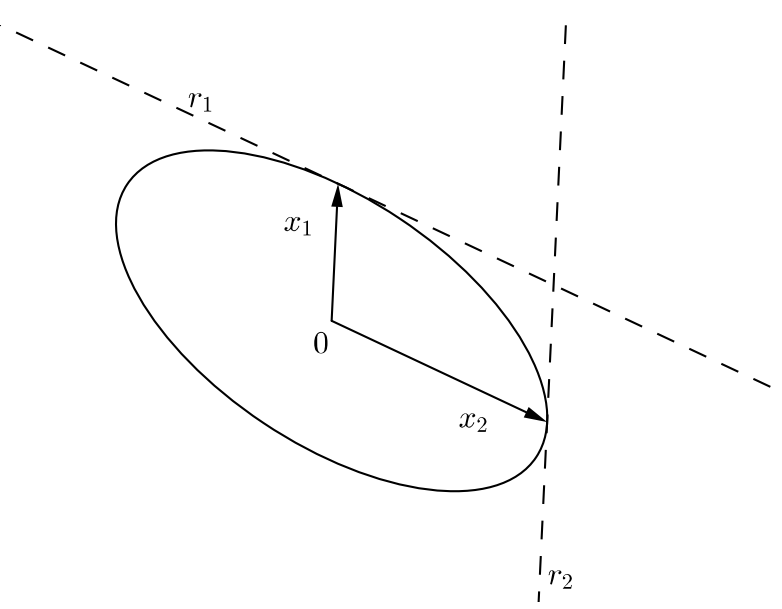

Figura 4.6: Um exemplo para o enunciado do teorema 4.7.

Prova. Considere dois pontos quaisquer $\left\{y_{1}, y_{2}\right\} \subseteq \mathcal{A}$ e denote por $A\left(\left\{y_{1}, y_{2}\right\}\right)$ a área do paralelogramo gerado por aqueles vetores. Como $\mathcal{A}$ é compacto e a área é uma função contínua, $A\left(\left\{y_{1}, y_{2}\right\}\right)$ atinge o valor máximo para algum conjunto $\left\{x_{1}, x_{2}\right\}$. Como $\mathcal{A}$ gera $\mathcal{M}$, a função área assume valores não-nulos e, portanto, seu máximo é positivo e vemos que o conjunto $\left\{x_{1}, x_{2}\right\}$ é linearmente independente. Sejam $r_{1}$ a reta $x_{1}+t x_{2}$ e $r_{2}$ a reta $x_{2}+t x_{1}$ para $t \in \mathbb{R}$. Assim, $r_{1}$ e $r_{2}$ são retas que passam por $x_{1}$ e $x_{2}$ e são paralelas a $\operatorname{span}\left\{x_{2}\right\}$ e $\operatorname{span}\left\{x_{1}\right\}$ respectivamente, satisfazendo (i) e (ii) (ver figura 4.6).

Suponha por absurdo, que a reta $r_{1}$ não tangencie $\mathcal{A}$ no ponto $x_{1}$. Então, existe um ponto $x_{1}^{\prime} \in \mathcal{A}$ no interior do lado oposto da reta $r_{1}$ a $x_{2}$. Então, se substituirmos $x_{1}$ por $x_{1}^{\prime}$, obtemos o conjunto $\left\{x_{1}^{\prime}, x_{2}\right\}$ tal que $A\left(\left\{x_{1}^{\prime}, x_{2}\right\}\right)>A\left(\left\{x_{1}, x_{2}\right\}\right)$, contradizendo o fato de $A\left(\left\{x_{1}, x_{2}\right\}\right)$ ser máximo de $A$.

Definição 4.7 Sejam $x, y \in \mathcal{M}$. Dizemos que $x$ é normal a y e denotamos por $x \dashv y$ quando

$$
\|x+\alpha y\| \geq\|x\|, \quad \forall \alpha \in \mathbb{R}
$$

Geometricamente a definição acima significa que $x \dashv y$ se, e somente se, a reta $x+\alpha y(\alpha \in \mathbb{R})$ tangencia a bola $B_{\|x\|}(0)$ em $x$.

Corolário 4.7.1 Existe uma base $\mathcal{B}=\left\{x_{1}, x_{2}\right\}$ tal que $\left\|x_{1}\right\|=\left\|x_{2}\right\|=1$, $x_{1} \dashv x_{2}$ e $x_{2} \dashv x_{1}$. Isto é, o par $x_{1}, x_{2}$ é mutuamente normal.

Prova. Seja $\left(x_{1}, x_{2}\right)$ um conjunto ordenado dado pelo teorema 4.7 e considere $\mathcal{A}=B$. Diretamente do teorema vemos que $r_{i}$ tangencia $B$ em $x_{i}$ para $i \in\{1,2\}$. Então, $\left\|x_{i}\right\|=1$ e, como $x_{1}+x_{2} \in r_{i}$, temos $x_{1} \dashv x_{2}$ e $x_{2} \dashv x_{1}$.

Corolário 4.7.2 Existem bases $\mathcal{B}=\left\{x_{1}, x_{2}\right\}$ de $\mathcal{M}$ e $\mathcal{B}^{*}=\left\{f_{1}, f_{2}\right\}$ de $\mathcal{M}^{*}$ tais que $\left\|x_{i}\right\|=\left\|f_{i}\right\|=1$ para todo $i \in\{1,2\}$ e $f_{i}\left(x_{j}\right)=\delta_{i j}$ para todo $i, j \in\{1,2\}$. 
Prova. Seja $\mathcal{B}=\left\{x_{1}, x_{2}\right\}$ uma base dada pelo corolário 4.7.1. Defina $f_{i}$ sobre $\mathcal{M}$ por $f_{i}\left(\alpha_{1} x_{1}+\alpha_{2} x_{2}\right)=\alpha_{i}$. Então, $\left\|x_{i}\right\|=1$ e $f_{i}\left(x_{j}\right)=\delta_{i j}$. Pelo teorema 4.7, existem as retas $r_{i}$ tangentes à $B$ em $x_{i}$ que, neste caso, são dadas por $r_{i}=\left\{x: f_{i}(x)=1\right\}, \operatorname{logo}\left\|f_{i}\right\|=1$.

Corolário 4.7.3 Existe um paralelogramo $\mathcal{C}$ com vértices $\left\{x_{1}+x_{2}, x_{1}-\right.$ $\left.x_{2},-x_{1}-x_{2},-x_{1}+x_{2}\right\}$ tal que $B \subseteq C$ e cada lado de $C$ tangencia $B$ no ponto médio de cada lado.

Prova. Tome a base $\mathcal{B}=\left\{x_{1}, x_{2}\right\}$ do corolário 4.7.1. Seja o paralelogramo $\mathcal{C}=\operatorname{conv}\left\{x_{1}+x_{2}, x_{1}-x_{2},-x_{1}-x_{2},-x_{1}+x_{2}\right\}$. Então, os lados que contém $x_{i}$ estão contidos nas retas $r_{i}$, que, portanto, tangenciam $B$ em $x_{i}$. A simetria de $B$ garante que o mesmo ocorre com os pontos $-x_{i}$ e as retas $-r_{i}$. Assim, as retas $\pm r_{i}$ envelopam $B$ e temos $B \subseteq C$. Evidentemente, pela construção de $\mathcal{C}$, cada $\pm x_{i}$ é ponto médio de cada lado de $\mathcal{C}$.

\section{4}

\section{0 problema isoperimétrico}

Seja $B \in \mathcal{M}$ um conjunto simétrico e estritamente convexo tal que a sua fronteira $\partial B$ seja uma curva suave. Conforme visto na seção anterior, $B$ determina a norma $\|.\|_{B}$ sobre $\mathcal{M}$ e se $u \in \partial B$ então qualquer $x \in \mathcal{M}$ pode ser escrito por $x=t u$ para algum $t \geq 0$. Assim, já vimos que $\|x\|_{B}=t$.

De acordo com a seção 2.5 e Craizer [10], $u$ pode ser parametrizado por $u(\theta)$, com $0 \leq \theta \leq 2 \pi$, tal que $u^{\prime}(\theta)$ seja um múltiplo não-negativo de $e_{\theta}$. Escrevemos

$$
u(\theta)=p(\theta) e_{r}+p^{\prime}(\theta) e_{\theta}
$$

em que $p(\theta)$ é a função suporte de $\partial B$. Note que, pela simetria de $B$, $u(\theta+\pi)=-u(\theta)$. Vemos que $u^{\prime}(\theta)=p^{\prime}(\theta) e_{r}+p(\theta) e_{\theta}+p^{\prime \prime}(\theta) e_{\theta}-p^{\prime}(\theta) e_{r}$. Logo,

$$
u^{\prime}(\theta)=\left(p(\theta)+p^{\prime \prime}(\theta)\right) e_{\theta}
$$

E também, por cálculos análogos aos do capítulo 3 para área mista, temos

$$
\left[u(\theta), u^{\prime}(\theta)\right]=p(\theta)\left(p(\theta)+p^{\prime \prime}(\theta)\right)
$$

De acordo com Alencar \& Santos [3], a curvatura de $u$ em $\theta$ é dada pela fórmula

$$
k(\theta)=\frac{\left[u^{\prime}(\theta), u^{\prime \prime}(\theta)\right]}{\left\|u^{\prime}(\theta)\right\|_{2}^{3}}
$$

e, fazendo mais algumas contas, vemos que $\left[u^{\prime}(\theta), u^{\prime \prime}(\theta)\right]=\left(p(\theta)+p^{\prime \prime}(\theta)\right)^{2}$.

Sabemos que uma região é estritamente convexa se, e somente se, sua fronteira é uma curva cujas curvaturas (não-nulas) têm o mesmo sinal em todo ponto. Este é o caso da bola $B$, então, como o sinal de $k(\theta)$ é dado por 
$\left[u^{\prime}(\theta), u^{\prime \prime}(\theta)\right]$, bastaria supor $p(\theta)+p^{\prime \prime}(\theta) \neq 0$, mas pela continuidade de $p$ e $p^{\prime \prime}$, iremos supor $p(\theta)+p^{\prime \prime}(\theta)>0$ para cada $\theta \in[0,2 \pi]$.

Sejam $\mathcal{B}=\left\{e_{1}, e_{2}\right\}$ a base de $\mathcal{M}$ e $\mathcal{B}^{*}=\left\{e_{1}^{*}, e_{2}^{*}\right\}$ a base dual de $\mathcal{M}^{*}$. Podemos identificar o plano $\mathcal{M}$ com seu dual $\mathcal{M}^{*}$. De fato, seja a função $\Lambda: \mathcal{M} \rightarrow \mathcal{M}^{*}$ definida por

$$
\Lambda(v)=f_{v}
$$

em que $f_{v}(w)=[w, v]$ para todo $w \in \mathcal{M}$. As propriedades dos determinantes garantem facilmente a linearidade de $\Lambda$. Além disso, $\Lambda$ é injetiva, pois se $\Lambda(v)=0$ (neste caso, 0 representa o funcional nulo), então $[w, v]=0$ para todo $w \in \mathcal{M}$. Suponha $v=(a, b)$ não-nulo. Podemos escolher $w=(b,-a)$ e $[w, v]=b^{2}+a^{2}$. Como pelo menos $a$ ou $b$ é diferente de zero, vemos que $[w, v] \neq 0$, o que é absurdo. Portanto, o núcleo de $\Lambda$ é $\{0\}$ e esta função é injetiva. Pelo teorema do núcleo e da imagem, segue imediatamente que $\Lambda$ também é sobrejetiva. Assim, concluímos que $\Lambda$ é um isomorfismo.

Por outro lado, conforme visto no exemplo 2.9, se escrevermos $f_{v}=$ $\left(\phi_{1}, \phi_{2}\right)$ na base $\mathcal{B}^{*}$ e se $v=(a, b) \in \mathcal{M}$, então $f_{v}\left(e_{1}\right)=\left[e_{1}, v\right]=b=\phi_{1}$ e $f_{v}\left(e_{2}\right)=\left[e_{2}, v\right]=-a=\phi_{2}$. Logo, $v=\left(-\phi_{2}, \phi_{1}\right)$ e isto significa que cada elemento de $\mathcal{M}^{*}$ é identificado com a rotação de $\pi / 2$ no sentido anti-horário do ponto correspondente em $\mathcal{M}$.

Lembramos que, se a norma dual de $\mathcal{M}^{*}$ é $\|f\|=\sup \{|f(w)|: w \in B\}$, com a identificação acima para cada $v \in \mathcal{M}$ temos

$$
\|v\|=\sup \{|[w, v]|: w \in B\}
$$

Definição $4.8 O$ isoperimetrix é a região $I_{B}$ definida por

$$
I_{B}=\Lambda^{-1}\left(B^{\circ}\right)
$$

Embora nesta seção estejamos considerando $B$ um conjunto estritamente convexo com fronteira suave, a definição acima também se aplica para bolas sem estas propriedades. Em palavras, o isoperimetrix é a imagem inversa da bola dual pela transformação $\Lambda$ e, pelo que vimos acima, ele é identificado com a bola dual após uma rotação de $\pi / 2$ no sentido anti-horário.

A nossa escolha para esta seção de uma bola $B$ estritamente convexa com fronteira suave possibilita determinar uma fórmula para a fronteira de $I_{B}$. Considere uma curva $v$ parametrizada por

$$
v(\theta)=\frac{u^{\prime}(\theta)}{\left[u(\theta), u^{\prime}(\theta)\right]}, \quad 0 \leq \theta \leq 2 \pi .
$$

Afirmamos que $v(\theta)$ é a parametrização da fronteira de $I_{B}$. De fato, dado 
$\theta \in[0,2 \pi],\left[u^{\prime}(\theta), v(\theta)\right]=0$ e vemos que as curvas de nível da função $f_{v(\theta)}$ são retas paralelas ao vetor $u^{\prime}(\theta)$. A convexidade de $B$ garante que as retas tangentes à $B$ envelopam $B$ e a simetria, que a bola $B$ esteja inteiramente contida na faixa determinada pelas duas retas tangentes à $B$ nos pontos $u(\theta)$ e $-u(\theta)$. Portanto, como $[u(\theta), v(\theta)]=1$, temos

$$
|[w, v(\theta)]| \leq[u(\theta), v(\theta)]=1, \quad \forall w \in B
$$

Isto significa que $\left\|f_{v(\theta)}\right\|=1$, isto é, $f_{v(\theta)} \in \partial B^{\circ}$ e, assim, a curva $v$ é identificada com a fronteira da bola dual como queríamos.

Pelas fórmulas 4-9 e 4-10, vemos que

$$
v(\theta)=\frac{1}{p(\theta)} e_{\theta}
$$

Assim, a simetria de $B$ garante a simetria de $I_{B}$, pois

$$
v(\theta+\pi)=\frac{1}{p(\theta+\pi)} e_{\theta+\pi}=\frac{1}{p(\theta)}\left(-e_{\theta}\right)=-v(\theta)
$$

Com poucas contas, concluímos também que

$$
\begin{gathered}
v^{\prime}(\theta)=-\frac{1}{p(\theta)} e_{r}-\frac{p^{\prime}(\theta)}{p^{2}(\theta)} e_{\theta} \\
{\left[v(\theta), v^{\prime}(\theta)\right]=p^{-2}(\theta)}
\end{gathered}
$$

Calculando a derivada em 4-14, temos

$$
v^{\prime \prime}(\theta)=\frac{2 p^{\prime}(\theta)}{p^{2}(\theta)} e_{r}+\left(-\frac{1}{p(\theta)}+\frac{2 p^{2}(\theta)}{p^{3}(\theta)}-\frac{p^{\prime \prime}(\theta)}{p^{2}(\theta)}\right) e_{\theta}
$$

E encontramos $\left[v^{\prime}(\theta), v^{\prime \prime}(\theta)\right]=\frac{p(\theta)+p^{\prime \prime}(\theta)}{p^{3}(\theta)}$. Esta expressão determina o sinal da curvatura de $v$. Por hipótese, $p(\theta)+p^{\prime \prime}(\theta)$ é positivo para todo $\theta$, logo a curva $v$ delimita uma região estritamente convexa. Além disso, a continuidade das funções $p, p^{\prime}$, seno e cosseno mostra que $v$ é uma curva suave.

Em suma, $I_{B}$ é um conjunto estritamente convexo, simétrico e fechado com interior não-vazio, cuja fronteira é suave. Portanto, a partir de agora, para nos auxiliar na demonstração do teorema 4.8, questão central deste trabalho, vamos considerar $I_{B}$ como uma bola unitária em $\mathcal{M}$ e a norma correspondente será denotada por $\|\cdot\|_{I_{B}}$. Neste caso, note que $I_{I_{B}}$ é identificada $\operatorname{com}\left(B^{\circ}\right)^{\circ}=B$ após uma rotação de $\pi$ no sentido anti-horário, mas como a bola $B$ é simétrica, esta rotação coincide com a bola $B$ original. Isto é, o isoperimetrix do isoperimetrix é a própria bola $B$.

Seja $\gamma$ uma curva fechada e suave que delimita uma região estritamente 
convexa $K$. Se $\gamma$ for parametrizada conforme a fórmula 2-25, então a fórmula para $\gamma^{\prime}(\theta)$ será análoga à 4-9 e podemos escrever $\gamma^{\prime}(\theta)=\lambda(\theta) v(\theta)$, em que

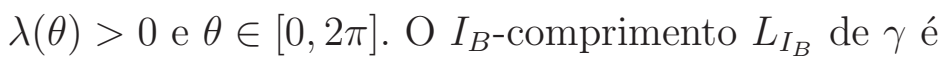

$$
L_{I_{B}}(\gamma)=\int_{0}^{2 \pi} \lambda(\theta) d \theta
$$

Pela fórmula 3-4, a área mista de $B$ e $K$ é dada por

$$
A(B, K)=\frac{1}{2} \int_{0}^{2 \pi}\left[u(\theta), \gamma^{\prime}(\theta)\right] d \theta=\frac{1}{2} \int_{0}^{2 \pi} \lambda(\theta)[u(\theta), v(\theta)] d \theta=\frac{1}{2} \int_{0}^{2 \pi} \lambda(\theta) d \theta
$$

Assim,

$$
A(B, K)=\frac{L_{I_{B}}(\gamma)}{2}
$$

Desta forma, se denotarmos por $A(B)$ e $A(K)$ as áreas de $B$ e de $K$ respectivamente, então a desigualdade de Minkowski (teorema 3.1) nos dá

$$
L_{I_{B}}^{2}(\gamma) \geq 4 A(B) A(K)
$$

Com igualdade se, e somente se, a região $K$ for homotética a $B$.

Teorema 4.8 Dentre todas as regiões estritamente convexas do plano $\mathcal{M}$ com perímetro $L_{0}$ fixado, cujas fronteiras são curvas suaves, aquela que possui a maior área é a homotética ao isoperimetrix.

Prova.

Existe $r>0$ tal que $L_{I_{B}}(r u)=L_{I_{B}}(\gamma)=L_{0}$. Denotaremos por $\tilde{B}$ a região $r B$. Por $4-17$, temos que $2 A(B)=2 A(B, B)=L_{I_{B}}(u)$. Como $L_{I_{B}}(r u)=r \cdot L_{I_{B}}(u)$, segue que $L_{I_{B}}(\gamma)=2 r A(B)$ e substituindo em 4-18 temos

$$
\begin{aligned}
4 r^{2} A^{2}(B) & \geq 4 A(B) A(K) \\
r^{2} A(B) & \geq A(K)
\end{aligned}
$$

Mas $A(\tilde{B})=r^{2} A(B)$ e concluímos

$$
A(\tilde{B}) \geq A(K)
$$

Com igualdade se, e somente se, $K=\tilde{B}$. Então, quando medimos o perímetro fixo de todas as regiões $K$ tendo como referência a bola $I_{B}$, a região de maior área é a homotética à bola $B$, que é o isoperimetrix de $I_{B}$, logo chegamos ao resultado desejado. 
Observação: O teorema acima é válido também para regiões cujas fronteiras sejam não suaves. O argumento é que estas podem ser aproximadas por regiões com fronteiras suaves.

Exemplo 4.6 Já sabemos que a solução para o problema isoperimétrico no plano euclideano é a bola, mas o teorema 4.8 diz que é a região homotética ao isoperimetrix. Como a norma dual da norma euclideana é a própria norma euclideana, vemos que neste caso $I_{B}=B$.

Exemplo 4.7 Mais geralmente, se $B$ for uma elipse teremos $I_{B}=\alpha B$ para algum $\alpha$ real. Dados $a, b \in \mathbb{R}$ e se $u \in \partial B$, então $u(\theta)=(a \cos \theta, b \operatorname{sen} \theta)$ para $\theta \in[0,2 \pi]$. Daí temos:

$$
\begin{gathered}
u^{\prime}(\theta)=(-a \operatorname{sen} \theta, b \cos \theta) \\
{\left[u(\theta), u^{\prime}(\theta)\right]=a b}
\end{gathered}
$$

E encontramos a parametrização de $\partial I_{B}$

$$
v(\theta)=\frac{1}{a b}(-a \operatorname{sen} \theta, b \cos \theta)
$$

Na figura 4.7 vemos que o isoperimetrix é uma elipse homotética a $B$, isto é, neste caso temos $I_{B}=\alpha B$ para algum $\alpha$ real.

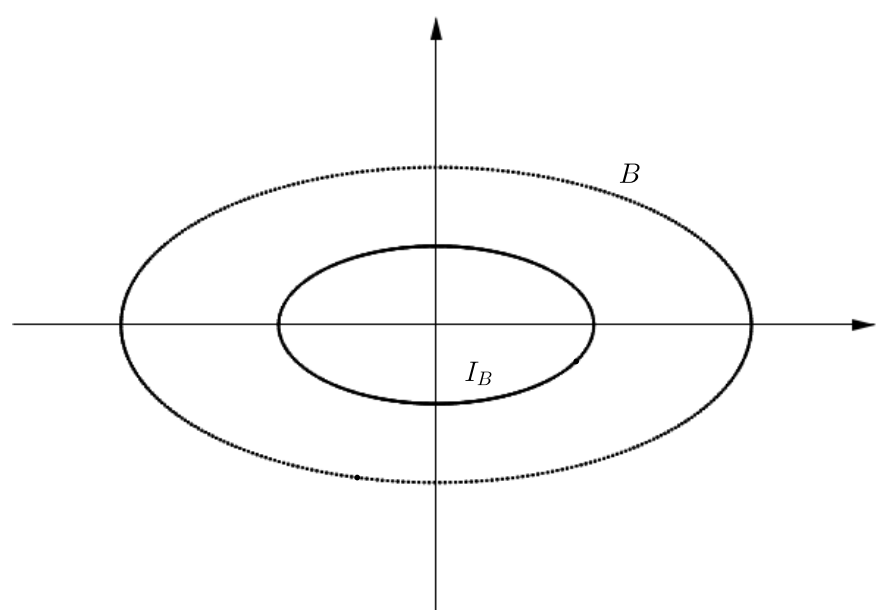

Figura 4.7: O isoperimetrix da elipse.

Na próxima seção, vamos investigar em quais casos o isoperimetrix coincide com a bola. 


\section{5}

\section{As curvas de Radon}

Nesta seção, seja $B$ um conjunto simétrico, convexo, limitado e fechado de $\mathcal{M}$ com interior não-vazio.

Seja $c_{0}$ o círculo unitário $\partial B$ com uma orientação. Pelo corolário 4.7.1, existe uma base $\mathcal{B}=\left\{x_{1}, x_{2}\right\}$ cujos vetores são mutuamente normais. Sejam $\mathcal{B}$ a base de $\mathcal{M}$ e $\mathcal{B}^{*}$ a base dual de $\mathcal{M}^{*}$.

Para cada funcional linear $f=\left(\phi_{1}, \phi_{2}\right) \in \partial B^{\circ}$ existe uma curva de nível de $f$ que tangencia $B$ em algum ponto $x \in c_{0}$. Se $t$ é um vetor tangente a $c_{0}$ em $x$, então $f(t)=0$ e a orientação de $c_{0}$ determina o sinal de $t$. Assim, o vetor $t$ é determinado por $t=-\phi_{2} x_{1}+\phi_{1} x_{2}=\left(-\phi_{2}, \phi_{1}\right)_{\mathcal{B}}$. Seja $c_{0}^{\prime}$ o conjunto dos vetores $t$ dado por

$$
c_{0}^{\prime}=\left\{\left(-\phi_{2}, \phi_{1}\right)_{\mathcal{B}}:\left(\phi_{1}, \phi_{2}\right)_{\mathcal{B}^{*}} \in \partial B^{\circ}\right\}
$$

Pela definição 4.8 , vemos que $c_{0}^{\prime}$ é a fronteira do isoperimetrix de $B$, isto é

$$
c_{0}^{\prime}=\Lambda^{-1}\left(\partial B^{\circ}\right)=\partial I_{B}
$$

em que $\Lambda$ é a função definida na seção anterior.

Pelo teorema 4.7, como os vetores da base $\mathcal{B}$ são mutuamente normais, existe uma tangente a $c_{0}$ em $x_{1}$ paralela a $x_{2}$ e outra tangente a $c_{0}$ em $x_{2}$ paralela a $-x_{1}$. Desta forma, podemos garantir que $c_{0}$ e $c_{0}^{\prime}$ passam pelos pontos $x_{1}, x_{2},-x_{1}$ e $-x_{2}$ e as duas curvas têm pelo menos uma tangente comum em cada um destes pontos. Assim, se $c_{1}=\left\{\left(\xi_{1}, \xi_{2}\right)_{\mathcal{B}} \in \partial B: \xi_{1} \geq 0, \xi_{2} \geq 0\right\}$ e $c_{1}^{\prime}=\left\{\left(-\phi_{2}, \phi_{1}\right)_{\mathcal{B}^{*}} \in \partial B^{\circ}: \phi_{1} \geq 0, \phi_{2} \geq 0\right\}$, podemos definir a curva $c_{0}^{\prime \prime}$ como

$$
c_{0}^{\prime \prime}=c_{1} \cup c_{1}^{\prime} \cup-c_{1} \cup-c_{1}^{\prime}
$$

Em outras palavras, a curva $c_{0}^{\prime \prime}$ é igual a $\partial B$ no primeiro e terceiro quadrantes e igual a $\partial I_{B}$ nos outros quadrantes. É claro que $c_{0}^{\prime \prime}$ é uma curva simétrica que limita uma região convexa. Uma curva construída de acordo com a definição 420 acima é chamada de curva de Radon.

Exemplo 4.8 Sejam $B$ a bola unitária $\ell_{1}$ e $B^{\circ}$ a bola dual correspondente, a bola $\ell_{\infty}$. A curva de Radon relativa a bola $B$ será a curva $R$ mostrada na figura 4.8 .

Pela construção de $c_{0}^{\prime \prime}$, é evidente que $\partial B$ é uma curva de Radon se, e somente se, $c_{0}=c_{0}^{\prime}$ e isto significa que, pela definição de $c_{0}^{\prime}, I_{B}=B$. 

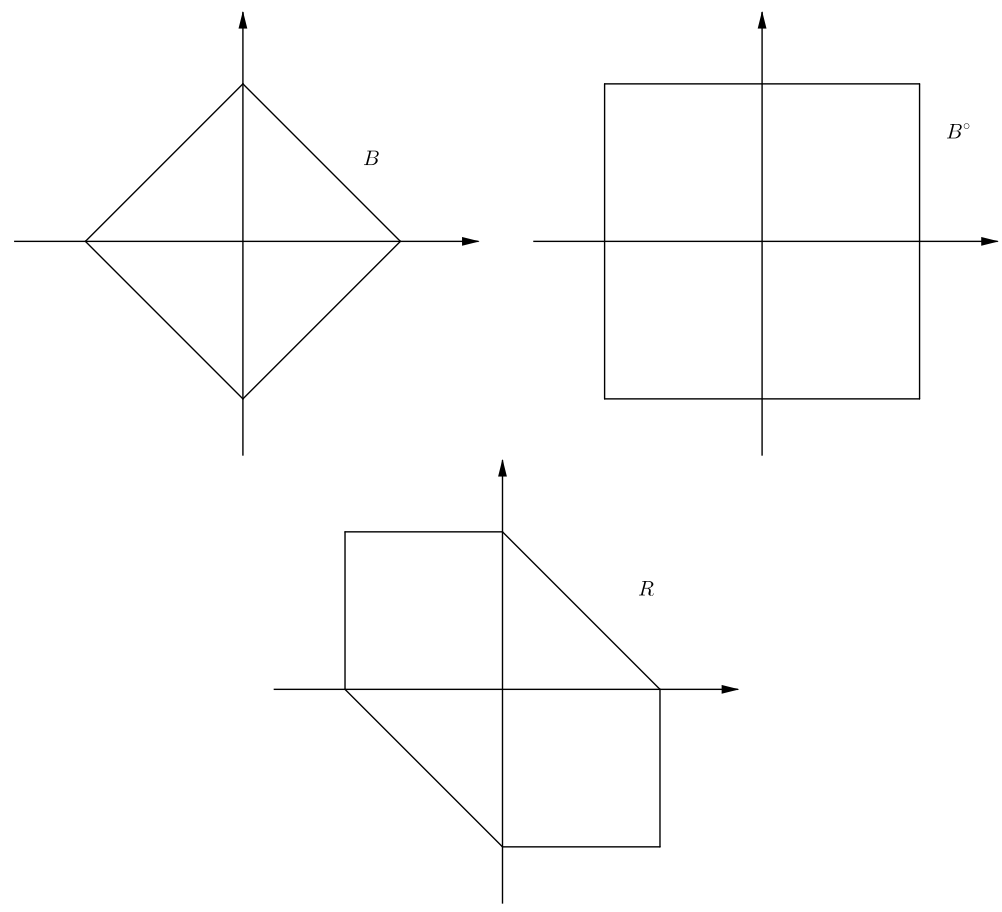

Figura 4.8: Construção de uma curva de Radon. 


\section{5 \\ O perímetro da bola unitária no plano de Minkowski}

Neste capítulo iremos estimar os possíveis valores que o perímetro da bola unitária pode assumir. Veremos que ele é mínimo quando $B$ for um hexágono regular afim e máximo quando $B$ for um paralelogramo. Para isto, em princípio veremos que no plano de Minkowski é sempre possível inscrever um hexágono de lados unitários em $B$ assim como acontece no plano euclideano (que é um caso particular de plano de Minkowski). Em seguida, daremos algumas definições relativas a curvas planas e demonstraremos alguns resultados que nos ajudarão a comparar comprimentos entre curvas inscritas e circunscritas. Assim como na seção 4.5, $B$ denotará um conjunto simétrico, convexo, limitado e fechado de $\mathcal{M}$ com interior não-vazio. A partir de agora, o símbolo $\|$. será usado para representar a norma $\|\cdot\|_{B}$. O conteúdo a seguir é baseado em Thompson [1].

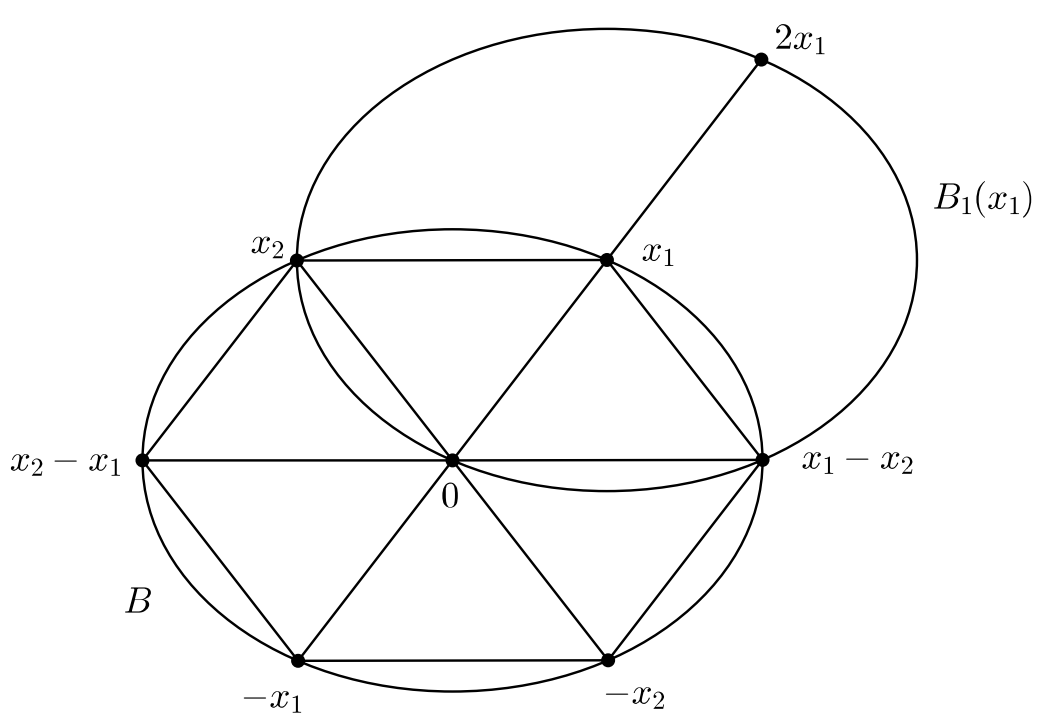

Figura 5.1: Construção de um hexágono de lados unitários.

Teorema 5.1 Se $x_{0} \in \mathcal{M}$, então existem $x_{1}, x_{2} \in \mathcal{M}$ tais que $\left\|x_{i}-x_{j}\right\|=1$ para $i, j=0,1,2$ e $i \neq j$. 
Prova. Sem perda de generalidade podemos supor $x_{0}=0$. Seja $x_{1}$ um ponto arbitrário de $\partial B$ conforme figura 5.1 , isto é, $\left\|x_{1}\right\|=1$. Considere a bola $B_{1}\left(x_{1}\right)$. A fronteira desta bola é uma curva que passa por 0 dentro de $\partial B$ e por $2 x_{1}$, que está fora de $\partial B$. Pela continuidade de $\partial B_{1}\left(x_{1}\right)$, evidentemente existe um ponto $x_{2} \in \partial B_{1}\left(x_{1}\right) \cap \partial B$. Então, $\left\|x_{2}\right\|=\left\|x_{2}-x_{1}\right\|=1 \mathrm{e}$, em resumo, temos

$$
\left\|x_{1}\right\|=\left\|-x_{1}\right\|=\left\|x_{2}\right\|=\left\|-x_{2}\right\|=\left\|x_{2}-x_{1}\right\|=\left\|x_{1}-x_{2}\right\|=1
$$

Portanto, os seis pontos $x_{1}, x_{2}, x_{2}-x_{1},-x_{1},-x_{2}$ e $x_{1}-x_{2}$ são os vértices de um hexágono regular inscrito em $B$.

Seja $c=\{x: x=\varphi(t)$ e $\alpha \leq t \leq \beta\}$ uma curva (ver seção 2.4) e se $\alpha=t_{0}<t_{1}<\ldots<t_{n}=\beta$ é uma partição do intervalo $[\alpha, \beta]$, então podemos considerar a poligonal cujos vértices são $\varphi\left(t_{0}\right), \varphi\left(t_{1}\right), \ldots$, $\varphi\left(t_{n}\right)$ com comprimento $\sum_{i=1}^{n}\left\|x_{i}-x_{i-1}\right\|$. Uma curva é dita retificável quando os comprimentos de tais poligonais são limitados superiormente. Assim, o comprimento da curva $c$, denotado por $L_{B}(c)$, pode ser definido também por

$$
L_{B}(c)=\sup \left\{\sum_{i=1}^{n}\left\|\varphi\left(t_{i}\right)-\varphi\left(t_{i-1}\right)\right\|:\left\{t_{0}, t_{1}, \ldots, t_{n}\right\} \text { é uma partição de }[\alpha, \beta]\right\}
$$

De acordo com Carmo [4], se $c$ é suave, a definição acima é equivalente à fórmula 2-10 da seção 2.4 .

Uma curva fechada e simples é dita convexa quando o interior dela é uma região convexa. Uma curva $c$ de $x_{0}$ a $x_{1}\left(x_{0} \neq x_{1}\right)$ é chamada de convexa quando $c \cup \overline{x_{1} x_{0}}$ é uma curva convexa, fechada e simples, em que $\overline{x_{1} x_{0}}$ denota o segmento de reta de extremos $x_{0}$ e $x_{1}$. Se $c_{1}$ e $c_{2}$ são duas curvas convexas de $x_{0}$ a $x_{1}$, então $c_{1}$ é dita interior à $c_{2}$ quando $c_{1} \subseteq \operatorname{conv} c_{2}$.

Lema 5.1 Se a poligonal $\wp$ de vértices $x_{0}, x_{1}, \ldots, x_{n}$ é uma curva convexa de $x_{0}$ a $x_{n}$ que seja interior à poligonal $\mathcal{P}$ cujos vértices são $x_{0}, y$ e $x_{n}$ (nesta ordem), então

$$
\sum_{i=1}^{n}\left\|x_{i}-x_{i-1}\right\| \leq\left\|y-x_{0}\right\|+\left\|x_{n}-y\right\|
$$

Prova. Suponhamos os vetores $u_{1}=y-x_{0}$ e $u_{2}=x_{n}-y$ linearmente independentes. O caso em que eles são linearmente dependentes é trivial. Podemos, então, considerar $\mathcal{B}=\left\{u_{1}, u_{2}\right\}$ uma base de $\mathcal{M}$ e cada vetor $x_{i}-x_{i-1}$ pode ser escrito

$$
x_{i}-x_{i-1}=\alpha_{i} u_{1}+\beta_{i} u_{2}
$$


Como $\wp$ é convexa e está no interior de $\mathcal{P}$, cada $\alpha_{i}$ e $\beta_{i}$ são não negativos. Então,

$x_{n}-x_{0}=\sum_{i=1}^{n}\left(x_{i}-x_{i-1}\right)=\sum_{i=1}^{n} \alpha_{i} u_{1}+\sum_{i=1}^{n} \beta_{i} u_{2}=\left(y-x_{0}\right)+\left(x_{n}-y\right)=u_{1}+u_{2}$

Assim, $\sum_{i=1}^{n} \alpha_{i}=\sum_{i=1}^{n} \beta_{i}=1$. Pela desigualdade triangular, $\left\|x_{i}-x_{i-1}\right\| \leq$ $\alpha_{i}\left\|u_{1}\right\|+\beta_{i}\left\|u_{2}\right\|$ e temos

$$
L_{B}(\wp)=\sum_{i=1}^{n}\left\|x_{i}-x_{i-1}\right\| \leq \sum_{i=1}^{n} \alpha_{i}\left\|u_{1}\right\|+\sum_{i=1}^{n} \beta_{i}\left\|u_{2}\right\|=\left\|u_{1}\right\|+\left\|u_{2}\right\|
$$

Teorema 5.2 Se c é uma curva convexa de $x_{0}$ a $x_{1}$ no interior da poligonal $\mathcal{P}$ de vértices $x_{0}, y$ e $x_{1}$ (ver figura 5.2), então

$$
L_{B}(c) \leq\left\|y-x_{0}\right\|+\left\|x_{1}-y\right\|
$$

Prova. Se $\wp$ é uma poligonal qualquer inscrita em $c$, então ela também é interior à $\mathcal{P}$. Pelo lema $5.1, L_{B}(\wp) \leq\left\|y-x_{0}\right\|+\left\|x_{1}-y\right\|$. Pela definição $5-1$, $L_{B}(c)=\sup \left\{L_{B}(\wp): \wp\right.$ é inscrito em $\left.c\right\}$, segue o resultado.

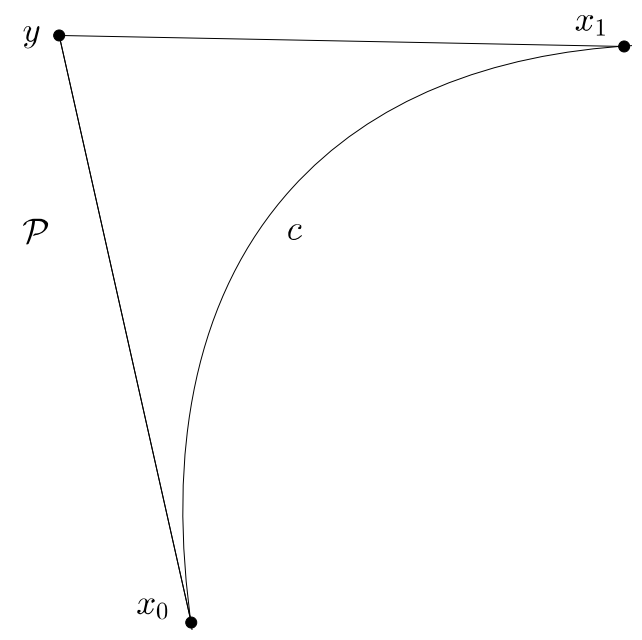

Figura 5.2: A curva $c$ está inscrita em $\mathcal{P}$.

Teorema 5.3 Se c é uma curva convexa de $x$ a y e se $\wp_{1}$ e $\wp_{2}$ são, respectivamente, poligonais inscritas e circunscritas à c de $x$ a $y$, então

$$
L_{B}\left(\wp_{1}\right) \leq L_{B}(c) \leq L_{B}\left(\wp_{2}\right)
$$


Prova. A primeira desigualdade é consequência direta da definição de $L_{B}(c)$. Seja $\wp_{2}$ uma poligonal cujos vértices são $x, y_{2}, \ldots, y_{n-1}$ e $y_{n}=y$ (nesta ordem). Ponha $y_{1}=y_{1}^{\prime}=x$ e $y_{n}=y_{n-1}^{\prime}=y$ e suponha que cada segmento de reta $\overline{y_{i} y_{i+1}}$ tangencia $c$ em $y_{i}^{\prime}$ para $i=2,3, \ldots, n-2$. Basta aplicar o teorema 5.2 para cada parte da curva $c$ de $y_{i}^{\prime}$ a $y_{i+1}^{\prime}$ e suas correspondentes poligonais circunscritas de vértices $y_{i}^{\prime}, y_{i+i}$ e $y_{i+1}^{\prime}$ para $i=1,2, \ldots, n-2$.

Definição 5.1 Um hexágono regular afim é a imagem de um hexágono regular por uma transformação afim.

É possível verificar as seguintes propriedades do hexágono regular afim (ver figura 5.3):

1. São paralelos: $A B, C F$ e $D E ; B C, A D$ e $E F ; C D, B E$ e $A F$.

2. As diagonais $A D, B E$ e $C F$ cruzam em um único ponto.

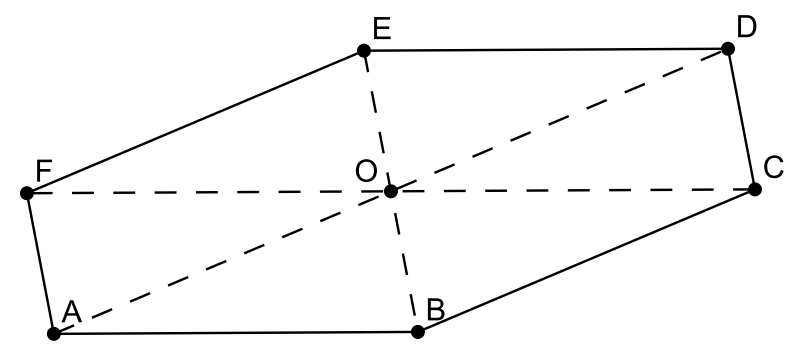

Figura 5.3: Hexágono regular afim.

Assim, se a bola unitária de $\mathcal{M}$ for um hexágono regular afim, então, pelas propriedades acima, vemos que o perímetro (de Minkowski) dela será 6 . Este fato é parte do teorema 5.5 que veremos mais adiante.

Teorema 5.4 (Golab)

$$
6 \leq L_{B}(\partial B) \leq 8
$$

Prova. O teorema 5.1 garante a existência de um hexágono inscrito em $B$ de lados unitários. Do teorema 5.3 segue imediatamente a primeira desigualdade. A segunda desigualdade é consequência do corolário 4.7.3, que afirma existir um paralelogramo $\wp_{2}=\operatorname{conv}\left\{x_{1}+x_{2}, x_{1}-x_{2},-x_{1}-x_{2},-x_{1}+x_{2}\right\}$ que contém $B$ e tal que $\left\|x_{1}\right\|=\left\|x_{2}\right\|=1$, donde os pontos $\pm x_{i}(i \in\{1,2\})$ são pontos médios dos lados. Logo, $L_{B}\left(\wp_{2}\right)=8$ e novamente aplicamos o teorema 5.3 para chegarmos ao resultado desejado. 


\section{Teorema 5.5}

(i) $L_{B}(\partial B)=6$ se, e somente se, $B$ é um hexágono regular afim;

(ii) $L_{B}(\partial B)=8$ se, e somente se, $B$ é um paralelogramo.

Prova. As "voltas" das afirmações acima são óbvias. As "idas" foram baseadas na prova de Schäffer e descritas por Thompson [1].

(i) Suponhamos $L_{B}(\partial B)=6$. Pelo teorema 5.1, podemos construir um hexágono regular inscrito em $B$ a partir de um vértice arbitrário $x_{1} \in \partial B$ (ver figura 5.4). Seja $x_{1}$ um ponto extremo de $B$. Denotemos por $c\left(x_{1} x_{2}\right)$ o arco de $\partial B$ que liga $x_{1}$ a $x_{2}$ no sentido anti-horário. Seja $y$ o ponto

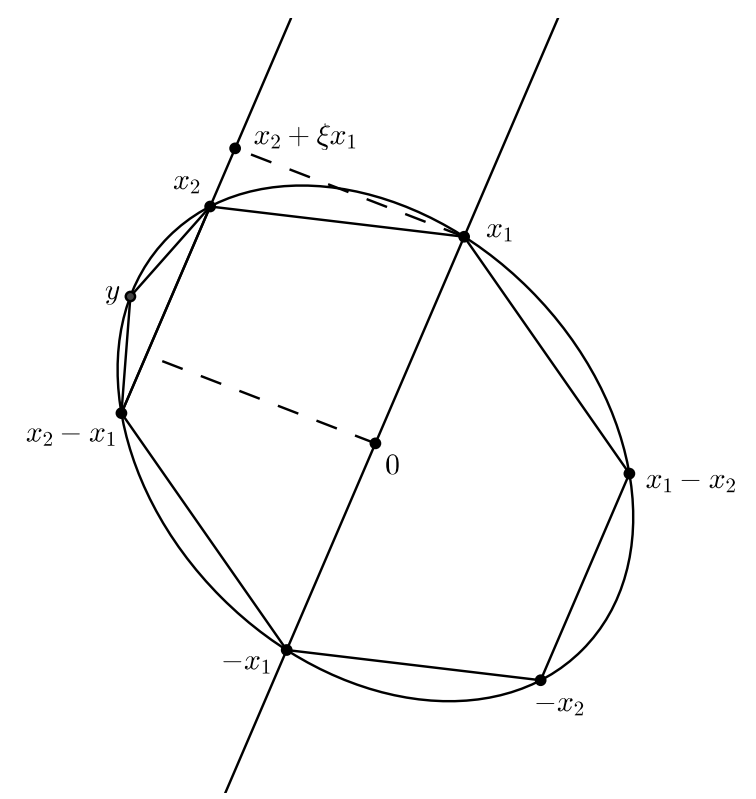

Figura 5.4: O hexágono está contido em $\partial B$.

médio do arco $c\left(x_{2}\left(x_{2}-x_{1}\right)\right)$ de $\partial B$. Então, pela desigualdade triangular e pelo teorema 5.3 , temos

$$
\begin{aligned}
3 & =\left\|x_{1}-x_{2}\right\|+\left\|x_{2}-\left(x_{2}-x_{1}\right)\right\|+\left\|\left(x_{2}-x_{1}\right)-\left(-x_{1}\right)\right\| \\
& \leq\left\|x_{1}-x_{2}\right\|+\left\|x_{2}-y\right\|+\left\|y-\left(x_{2}-x_{1}\right)\right\|+\left\|\left(x_{2}-x_{1}\right)-\left(-x_{1}\right)\right\| \\
& \leq\left\|x_{1}-x_{2}\right\|+L_{B}\left(c\left(x_{2} y\right)\right)+L_{B}\left(c\left(y\left(x_{2}-x_{1}\right)\right)\right)+\left\|\left(x_{2}-x_{1}\right)-\left(-x_{1}\right)\right\| \\
& \leq \frac{L_{B}(\partial B)}{2}=3
\end{aligned}
$$

Donde vemos que

$$
\left\|x_{2}-y\right\|+\left\|y-\left(x_{2}-x_{1}\right)\right\|=L_{B}\left(c\left(x_{2} y\right)\right)+L_{B}\left(c\left(y\left(x_{2}-x_{1}\right)\right)\right)=1
$$

Pela escolha de $y$, temos $L_{B}\left(c\left(x_{2} y\right)\right)=L_{B}\left(c\left(y\left(x_{2}-x_{1}\right)\right)\right)=1 / 2$, o que implica $\left\|x_{2}-y\right\| \leq 1 / 2$ e $\left\|y-\left(x_{2}-x_{1}\right)\right\| \leq 1 / 2$. Se fosse possível $\left\|x_{2}-y\right\|<$ 
$1 / 2$ ou $\left\|y-\left(x_{2}-x_{1}\right)\right\|<1 / 2$, teríamos $\left\|x_{2}-y\right\|+\left\|y-\left(x_{2}-x_{1}\right)\right\|<1$, o que é absurdo. Portanto, $\left\|x_{2}-y\right\|=\left\|y-\left(x_{2}-x_{1}\right)\right\|=1 / 2$, donde $2\left(x_{2}-y\right) \in \partial B$ e $2\left(y-\left(x_{2}-x_{1}\right)\right) \in \partial B$. Por outro lado, $x_{1}$ pode ser escrito como

$x_{1}=\left(x_{2}-y\right)+\left(y-\left(x_{2}-x_{1}\right)\right)=\frac{1}{2} \cdot\left[2\left(x_{2}-y\right)\right]+\frac{1}{2} \cdot\left[2\left(y-\left(x_{2}-x_{1}\right)\right)\right]$

Como $x_{1}$ é um ponto extremo de $B$, pelas definições 2.3 e 2.4 , temos que $x_{1}=2\left(x_{2}-y\right)=2\left(y-\left(x_{2}-x_{1}\right)\right)$. Assim,

$$
y=x_{2}-\frac{1}{2} x_{1}=\frac{x_{2}+\left(x_{2}-x_{1}\right)}{2}
$$

e verificamos que $y$ é ponto médio do segmento $\overline{x_{2}\left(x_{2}-x_{1}\right)}$. Além disso, como os pontos $x_{2}, y$ e $x_{2}-x_{1}$ pertencem à $\partial B$, podemos dizer que o segmento de reta $\overline{x_{2}\left(x_{2}-x_{1}\right)} \subseteq \partial B$, pois caso contrário, pela convexidade de $B$, existiria um ponto $w$ (digamos entre $y$ e $x_{2}-x_{1}$ ) do segmento $\overline{x_{2}\left(x_{2}-x_{1}\right)}$ no interior de $B$ conforme mostra figura 5.5, então poderíamos prolongar o segmento $0 w$ até encontrar $\partial B$ no ponto $z$ e assim construiríamos o triângulo $x_{2} 0 z$ inteiramente contido em $B$ (novamente pela convexidade) e o ponto $y$ pertenceria ao interior deste triângulo, o que é absurdo.

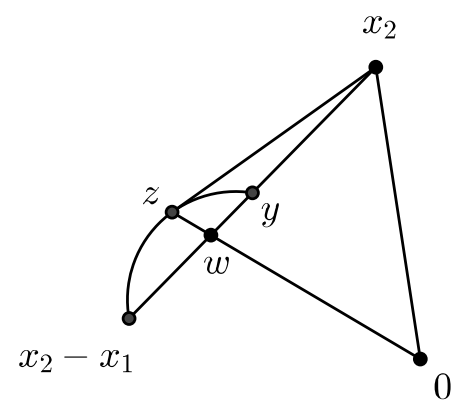

Figura 5.5: $\overline{x_{2}\left(x_{2}-x_{1}\right)} \subseteq \partial B$.

Agora vamos provar que o ponto $x_{2}$ é um ponto extremo de $B$. Suponhamos por absurdo que não, então existe $\xi \in(0,1)$ tal que $x_{2}+\xi x_{1} \in \partial B$. Assim, como $\overline{x_{2}\left(x_{2}-x_{1}\right)} \subseteq \partial B$, vemos que

$$
\left\|\left(x_{2}+\xi x_{1}\right)-x_{1}\right\|=\left\|\xi x_{2}+(1-\xi)\left(x_{2}-x_{1}\right)\right\|=1
$$


segue que

$$
\begin{aligned}
\frac{L_{B}(\partial B)}{2} \geq & \left\|\left(x_{2}+\xi x_{1}\right)-x_{1}\right\|+\left\|\left(x_{2}+\xi x_{1}\right)-\left(x_{2}-x_{1}\right)\right\| \\
& +\left\|\left(x_{2}-x_{1}\right)-\left(-x_{1}\right)\right\| \\
= & 1+(1+\xi)+1>3
\end{aligned}
$$

e chegamos a um absurdo. Portanto, $x_{2}$ é um ponto extremo de $B$ e podemos repetir a prova acima iniciando por $x_{2}$. Enfim, analogamente podemos continuar o processo, provando que todos os vértices do hexágono inscrito são pontos extremos de $B$ e consequentemente que todos os seus lados estão contidos em $\partial B$.

(ii) Suponhamos $L_{B}(\partial B)=8$. Pelo corolário 4.7.3, existe um paralelogramo $\wp$ de vértices $\left\{x_{1}+x_{2}, x_{1}-x_{2},-x_{1}-x_{2},-x_{1}+x_{2}\right\}$ circunscrito a $B$ com $\left\|x_{1}\right\|=\left\|x_{2}\right\|=1$ conforme mostra a figura 5.6. Por um argumento análogo ao do item anterior, se cada um dos vértices do paralelogramo pertencerem à $\partial B$, como os pontos médios $x_{1},-x_{1}, x_{2}$ e $-x_{2}$ dos lados também pertencem à $\partial B$, então podemos garantir que cada lado está contido em $\partial B$, donde $\partial B=\wp$ e não há mais nada a fazer neste caso.

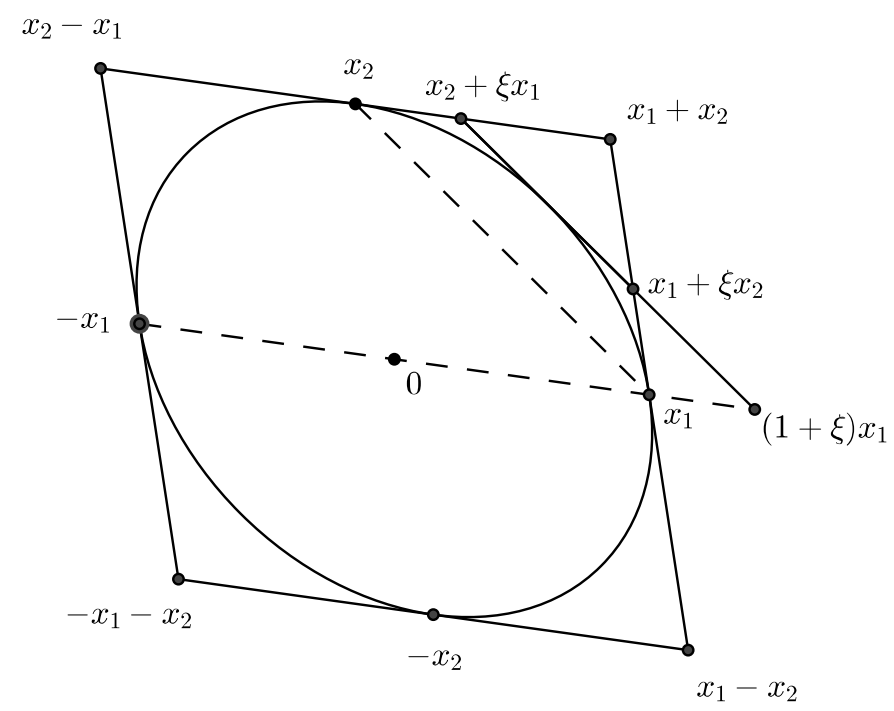

Figura 5.6: O paralelogramo está contido em $\partial B$.

Suponhamos agora que $x_{1}+x_{2} \notin \partial B$ (e, pela simetria, também $-x_{1}-x_{2} \notin$ $\partial B)$, então existe $\xi \in[0,1)$ tal que a reta $\left\{\left(x_{2}-x_{1}\right) t+(1+\xi) x_{1}: t \in \mathbb{R}\right\}$ seja tangente a $B$. É fácil verificar que esta reta corta os lados de $\wp \mathrm{em}$ $x_{1}+\xi x_{2}$ e $x_{2}+\xi x_{1}$ como mostra a figura 5.6. Então, pelo teorema 5.3, 
temos

$$
\begin{aligned}
4=\frac{L_{B}(\partial B)}{2} \leq & \left\|\left(x_{1}+\xi x_{2}\right)-x_{1}\right\|+\left\|\left(x_{2}+\xi x_{1}\right)-\left(x_{1}+\xi x_{2}\right)\right\| \\
& +\left\|\left(x_{2}-x_{1}\right)-\left(x_{2}+\xi x_{1}\right)\right\|+\left\|-x_{1}-\left(x_{2}-x_{1}\right)\right\| \\
= & \xi+(1-\xi)\left\|x_{2}-x_{1}\right\|+(1+\xi)+1 \\
= & 2+2 \xi+(1-\xi)\left\|x_{2}-x_{1}\right\| \\
\leq & 2+2 \xi+2(1-\xi)=4
\end{aligned}
$$

pois $\left\|x_{2}-x_{1}\right\| \leq\left\|x_{2}\right\|+\left\|x_{1}\right\|=2$ implica $(1-\xi)\left\|x_{2}-x_{1}\right\| \leq 2(1-\xi)$. Isto é,

$$
4 \leq 2+2 \xi+(1-\xi)\left\|x_{2}-x_{1}\right\| \leq 4
$$

Logo, $2 \xi+(1-\xi)\left\|x_{2}-x_{1}\right\|=2$ e, como $\xi<1$, temos

$$
\left\|x_{2}-x_{1}\right\|=\frac{2(1-\xi)}{(1-\xi)}=2
$$

Assim, vemos que o ponto médio do segmento $\overline{-x_{1} x_{2}}$, que é o ponto $\frac{1}{2}\left(x_{2}-x_{1}\right)$, pertence à $\partial B$ e o vértice $x_{2}-x_{1}$ não (pela simetria, $-x_{2}+x_{1}$ também não). Como os pontos $-x_{1}$ e $x_{2}$ também pertencem à fronteira de $B$, vemos que o segmento $\overline{-x_{1} x_{2}} \subseteq \partial B$. Podemos repetir o processo acima para cada um dos vértices $x_{2}-x_{1},-x_{2}+x_{1}$ e $-x_{1}-x_{2}$ (que, conforme vimos, não pertencem à $\partial B$ ) para mostrar que os segmentos $\overline{\left(-x_{1}\right)\left(-x_{2}\right)}, \overline{-x_{2} x_{1}}$ e $\overline{x_{1} x_{2}}$ estão contidos em $\partial B$, donde concluímos que $B$ é o paralelogramo de vértices $x_{1},-x_{1}, x_{2} \mathrm{e}-x_{2}$. Não tendo mais outros casos, concluímos nossa prova.

Exemplo 5.1 Seja a bola $B$ definida por

$$
B=\operatorname{conv}\{(1,1),(-\xi, 1),(-1, \xi),(-1,-1),(\xi,-1),(1,-\xi)\}, \operatorname{com} \xi \in[0,1]
$$

conforme mostra a figura 5.7. Para cada $\xi \in[0,1), B$ é um hexágono e se $\xi=1, B$ é um quadrado (ou, se quiser, um hexágono degenerado). Note que os lados paralelos aos eixos têm comprimento (relativo à $B$ ) igual a $1+\xi e$ cada um dos outros dois lados medem $\|(1,-\xi)-(\xi,-1)\|=\|(1-\xi,-\xi+1)\|=$ $(1-\xi)\|(1,1)\|=(1-\xi)$. Logo,

$$
L_{B}(\partial B)=6+2 \xi, \quad \text { para } \xi \in[0,1]
$$




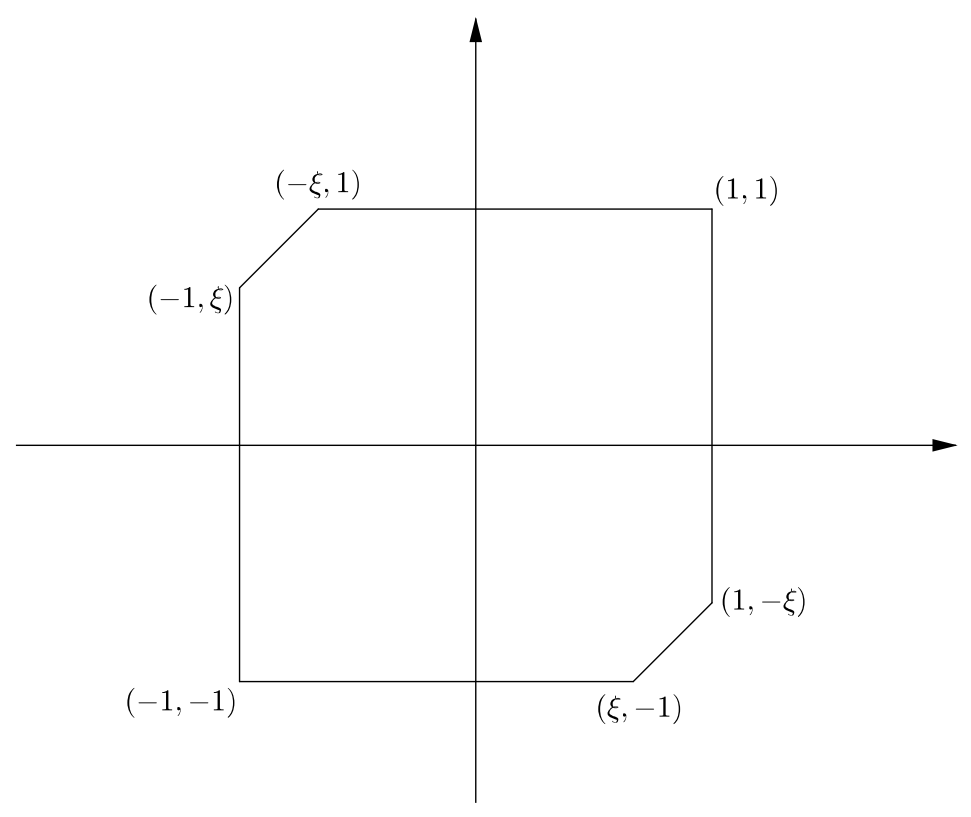

Figura 5.7: Exemplo de bola cujo perímetro é $6+2 \xi$.

O teorema 5.4 afirma que qualquer bola no plano de Minkowski tem perímetro mínimo seis e no máximo oito. Mas o exemplo 5.1 mostra que o perímetro de $B$ pode assumir qualquer valor real entre 6 e 8, sendo 6 quando $B$ é um hexágono regular afim e 8 quando $B$ é um paralelogramo, conforme prevê o teorema 5.5. Assim, na geometria de Minkowski se mantivermos a definição do número $\pi$ como sendo a razão entre o perímetro da bola e seu diâmetro, $\pi$ poderá assumir qualquer valor real no intervalo [3, 4] dependendo da escolha de $B$. 


\section{6 \\ Referências Bibliográficas}

1 THOMPSON, A. C. Minkowski Geometry. Cambridge: Press Syndicate of the University of Cambridge, 1996. (Encyclopedia of mathematics and its applications, v. 63).

2 MARTINI, H.; WU, S. Classical curve theory in normed planes. Computer Aided Geometric Design, 2014.

3 ALENCAR, H.; SANTOS, W. Geometria Diferencial das Curvas Planas. Rio de Janeiro: Publicações Matemáticas do IMPA, 2003.

4 CARMO, M. P. do. Geometria Diferencial de Curvas e Superfícies. 4. ed. Rio de Janeiro: SBM, 2010. (Coleção Textos Universitários). ISBN 978-85-85818-26-5.

5 BUSEMANN, H. The isoperimetric problem in the minkowski plane. American Journal of Mathematics, v. 69, n. 4, p. 863-871, 1947.

6 STRANG, G. Maximum area with minkowski measures of perimeter. Proceedings of the Royal Society of Edinburgh, v. 138A, p. 189199, 2008.

7 LIMA, E. L. Álgebra Linear. 7. ed. Rio de Janeiro: IMPA, 2004. (Coleção Matemática Universitária). ISBN 85-244-0089-7.

8 LIMA, E. L. Curso de Análise. 11. ed. Rio de Janeiro: IMPA, 2011. (Projeto Euclides, v. 2). ISBN 978-85-244-0049-0.

9 FLANDERS, H. A proof of minkowski's inequality for convex curves. The American Mathematical Monthly, v. 75, n. 6, p. 581-593, Jun. - Jul. 1968.

10 CRAIZER, M. Iteration of involutes of constant width curves in the minkowski plane. Beiträge zur Algebra und Geometrie, v. 55, n. 2, p. 479-496, 2014. 